\title{
Note on the Labelled tree graphs
}

\author{
Bo Feng $^{a, b}$ and Yaobo Zhang ${ }^{a}$ \\ ${ }^{a}$ Zhejiang Institute of Modern Physics, Department of Physics, Zhejiang University, \\ No. 38 Zheda Road, Hangzhou 310027, P.R. China \\ ${ }^{b}$ Center of Mathematical Science, Zhejiang University, \\ No. 38 Zheda Road, Hangzhou 310027, P.R. China \\ E-mail: fengbo@zju.edu.cn, yaobozhang@zju.edu.cn
}

ABSTRACT: In the CHY-frame for the tree-level amplitudes, the bi-adjoint scalar theory has played a fundamental role because it gives the on-shell Feynman diagrams for all other theories. Recently, an interesting generalization of the bi-adjoint scalar theory has been given in [1] by the "Labelled tree graphs", which carries a lot of similarity comparing to the bi-adjoint scalar theory. In this note, we have investigated the Labelled tree graphs from two different angels. In the first part of the note, we have shown that we can organize all cubic Feynman diagrams produces by the Labelled tree graphs to the "effective Feynman diagrams". In the new picture, the pole structure of the whole theory is more manifest. In the second part, we have generalized the action of "picking pole" in the bi-adjoint scalar theory to general CHY-integrands which produce only simple poles.

Keywords: Scattering Amplitudes, Differential and Algebraic Geometry

ArXIV EPRINT: 2009.02394 


\section{Contents}

1 Introduction 1

2 The effective Feynman diagrams $\quad 5$

2.1 Two basic types of effective Feynman vertexes 5

2.2 The example of next-to-Star graph 8

2.3 Enumerate Feynman diagrams from an effective Feynman diagram 13

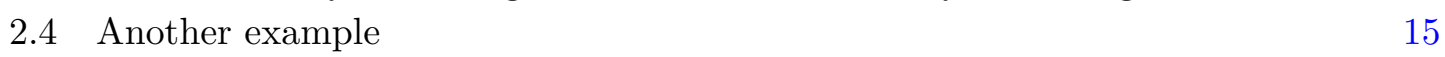

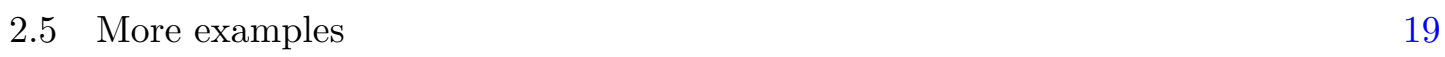

2.6 Application of effective Feynman diagrams 21

3 Pick up Poles $\quad 24$

3.1 The General algorithm 26

3.2 Examples 31

$\begin{array}{lll}3.2 .1 & \text { The simplest case } & 31\end{array}$

3.2.2 The next simplest examples 33

$\begin{array}{lll}3.2 .3 & \text { More complicated examples } & 34\end{array}$

3.3 The pick-factors with mixed type of cross-ratio factors 36

4 Conclusion $\quad 45$

\section{Introduction}

In 2013, a remarkable formula of tree-level amplitudes for massless particles has been proposed in $[2-6]$, which is written as

$$
\mathcal{A}_{n}=\int \frac{\left(\prod_{i=1}^{n} d z_{i}\right)}{\operatorname{vol}(S L(2, \mathbb{C}))} \Omega(\mathcal{E}) \mathcal{I}(z)=\int \frac{\left(\prod_{i=1}^{n} d z_{i}\right)}{d \omega} \Omega(\mathcal{E}) \mathcal{I}
$$

where $z_{i}$ are puncture locations of $n$ external particles in $\mathbb{C P}^{1}$ and $d \omega=\frac{d z_{r} d z_{s} d z_{t}}{z_{r s} z_{s t} z_{t r}}$ comes from the gauge fixing of the Möbius symmetry $S L(2, \mathbb{C})$ at the locations of three variables $z_{r}, z_{s}, z_{t}$ using the Faddeev-Popov method. The $\Omega$ is given by

$$
\Omega(\mathcal{E}) \equiv z_{i j} z_{j k} z_{k i} \prod_{a \neq i, j, k} \delta\left(\mathcal{E}_{a}\right)
$$

where $\mathcal{E}_{a}$ 's are the famous scattering equations defined as

$$
\mathcal{E}_{a} \equiv \sum_{b \neq a} \frac{s_{a b}}{z_{a}-z_{b}}=0, \quad a=1,2, \ldots, n .
$$

In the CHY-formula (1.1), there are two parts. The first part is the universal measure part $\frac{\left(\prod_{i=1}^{n} d z_{i}\right)}{d \omega} \Omega(\mathcal{E})$. The second part is the special part, i.e., the so called "CHY-integrand" $\mathcal{I}$, 
which defines the special theory. As a function of $z_{i}{ }^{\prime}$ 's, for (1.1) to be well defined, the CHY integrand $\mathcal{I}$ must transform covariantly under an $S L(2, \mathbb{C})$ transformation, with opposite weight as $d \omega$

$$
\begin{aligned}
z_{i} & \rightarrow \frac{\alpha z_{i}+\beta}{\gamma z_{i}+\delta} & \alpha \delta-\beta \gamma=1 \\
\mathcal{I}_{n} & \rightarrow \prod_{i=1}^{n}\left(\gamma z_{i}+\delta\right)^{4} \mathcal{I}_{n}\left(\left\{z_{i}\right\}\right) &
\end{aligned}
$$

Such a condition in (1.4) is called the weight four condition for CHY-integrands. The CHY-integrand of a given theory is, in general, a sum of rational functions of $z_{i j} \equiv z_{i}-z_{j}$. If we use a solid line (or a dashed line) to represent a factor $z_{i j}$ in the denominator (or the numerator), the weight four condition for each term can be rephrased as the 4-regular graph, i.e., a graph such that for each node, the number of attached solid lines minus the number of attached dashed lines is four.

For theories with $n$ particles, there are $(n-3)$ ! solutions to these scattering equations. It is well known that when $n>5$, it is impossible to have analytical solutions and one need to apply numerical method. However, without analytic results, many theoretical studies will be difficult. There are several works, where the analytic solutions have been bypassed and final analytic results can be obtained using computational algebraic geometry method like [7-13], such as the companion matrix, the Bezoutian matrix, the elimination theorem. In [14-16], integration rule has been proposed to read out Feynman diagrams for CHY-integrands containing only simple poles analytically without solving the scattering equations. For more general CHY-integrands with higher poles, we can use the cross ratio identities [17] to reduce the degree of poles one by one until all poles are simple poles, then the integration rule can be applied.

Now we briefly review how to read out the analytical expression for general weight four CHY-integrands. To do so, an important quantity we need to calculate is the pole index of each subset $A_{i} \subset\{1,2, \ldots, n\}$, which is defined as ${ }^{1}$

$$
\chi\left(A_{i}\right) \equiv L\left[A_{i}\right]-2\left(\left|A_{i}\right|-1\right),
$$

where $\left|A_{i}\right|$ is the number of external particles inside the subset $A_{i}$ and $L\left[A_{i}\right]$ is the linking number which is given by

$$
L\left[A_{i}\right]=\sum_{a<b ; a, b \in A_{i}} \beta_{a b}
$$

with the CHY-integrand given by $\mathcal{I}=\prod_{1 \leq a<b \leq n} z_{a b}^{-\beta_{a b}}$. For a given subset $A$ with the pole index $\chi[A] \geq 0$, the amplitude could have terms with poles like $\frac{1}{s_{A_{i}}^{\chi\left[A_{i}\right]+1}}$, where

$$
s_{A_{i}}=\left(\sum_{a \in A_{i}} k_{a}\right)^{2}=\left(\sum_{b \in \bar{A}_{i}} k_{b}\right)^{2} .
$$

\footnotetext{
${ }^{1}$ It is worth to notice that the subset $A_{i}$ and its complement subset $\bar{A}_{i}$ have the same pole index by the weight four condition for CHY-integrands.
} 


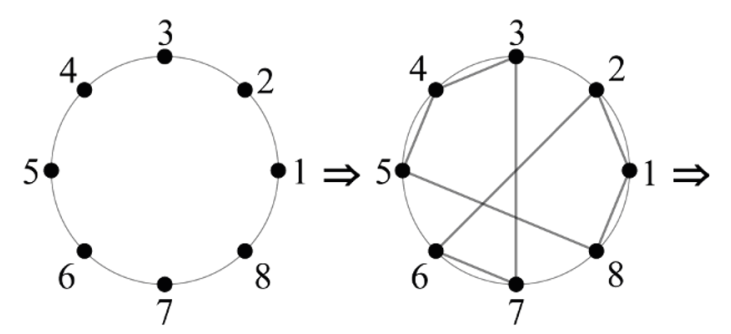

(a)

(b)

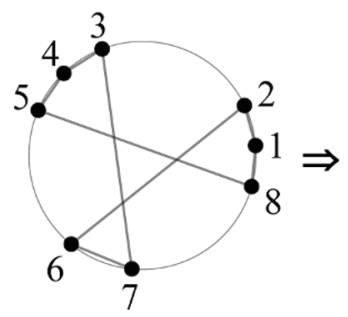

(c)

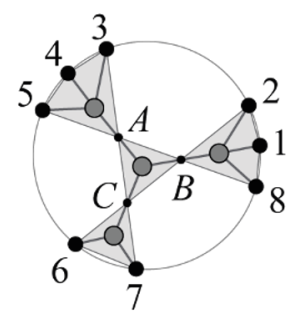

(d)

Figure 1. The procedure to obtain the effective Feynman diagram of $I[(12345678) \mid(12673458)]$ and the corresponding cubic Feynman diagrams are $\frac{1}{s_{128}} \frac{1}{s_{345}} \frac{1}{s_{67}}\left(\frac{1}{s_{12}}+\frac{1}{s_{18}}\right)\left(\frac{1}{s_{34}}+\frac{1}{s_{45}}\right)$.

When $\chi[A]>0$, it is higher pole. When CHY-integrand contains higher poles, as we have mentioned, a good way to deal with them is to use the cross ratio identity to translate the given CHY-integrands to the sum of CHY-integrands having only simple poles, i.e., $\chi[A] \leq 0, \forall A$. Thus the basis of the analytic method is the study of CHY-integrands with only simple poles. A well studied example is the bi-adjoint scalar theory with CHYintegrands

$$
I[\pi \mid \rho]=P T(\pi) \times P T(\rho)
$$

where $\pi, \rho$ are two orderings of external particles and for the given ordering $\alpha$, the ParkTaylor factor is defined by

$$
P T(\pi) \equiv \frac{1}{\left(z_{\pi(1)}-z_{\pi(2)}\right)\left(z_{\pi(2)}-z_{\pi(3)}\right) \cdots\left(z_{\pi(n-1)}-z_{\pi(n)}\right)\left(z_{\pi(n)}-z_{\pi(1)}\right)} .
$$

A good property of (1.8) is that the analytic expression is the sum of certain cubic tree Feynman diagrams with the same sign. For the bi-adjoint scalar theory, a diagrammatic method has been suggested in [3], which can be summarized as the effective Feynman diagrams [18]. As showing in figure 1, having the effective Feynman diagram, we could easily read out the analytic expression by noticing that each vertex with $m$ legs represents the sum of all ordered cubic Feynman diagrams of $m$ external legs.

Bi-adjoint scalar theory is very important in the CHY-frame. Just like the Feynman diagrams in the quantum field theory, the amplitudes of bi-PT integrands provides the on-shell cubic Feynman diagrams in the CHY-frame for all other theories, so amplitudes of other theories can be expanded by bi-adjoint scalar amplitudes with kinematic numerators as coupling constant of these on-shell cubic Feynman diagrams. Bi-adjoint scalar theory has also the simplest CHY-integrands, i.e., as the products of two PT-factor, thus many properties of this theory can be easily abstracted. Thus it is very natural to ask how many good behaviors of bi-adjoint scalar theory can be generalized to other CHY-integrands.

Recently, in [1], Gao, He and Zhang have given a new class of weight two CHYintegrands (the so called "Cayley functions"), which largely generalized the Parke-Taylor factor (1.9). The Cayley functions could be understood better in the gauge fixed form $z_{n} \rightarrow \infty$. With this gauge fixing, each Cayley function is mapped to a labelled tree 
graph with $(n-1)$-nodes $\{1,2, \ldots, n-1\}$ connecting by $(n-2)$ edges $^{2}$ (we will call them "Cayley tree" $\left.T_{n-1}\right)$. From a Cayley tree, we can read out the corresponding gauge fixed weight two CHY-integrand as

$$
C_{n}\left(T_{n-1}\right):=\frac{1}{\prod_{\{i, j\} \in \operatorname{Edges}\left(T_{n-1}\right)}\left(z_{i}-z_{j}\right)}
$$

or the $S L(2, C)$ covariant form

$$
C_{n}\left(T_{n-1}\right):=\frac{\prod_{k \in \operatorname{Vertexes}\left(T_{n-1}\right)}\left(z_{k}-z_{n}\right)^{v_{k}-2}}{\prod_{\{i, j\} \in \operatorname{Edges}\left(T_{n-1}\right)}\left(z_{i}-z_{j}\right)}
$$

when we put the $z_{n}$ back. As shown in [1], when applying the integration rule method, like the PT-factor (1.9), the combinations of Cayley tree's produce also the sum of certain cubic tree Feynman diagrams with the same sign. Furthermore, they showed how all these diagrams can be constructed by iterative algorithm. These diagrams can be combinatorially organized to a geometric picture, i.e., the "combinatoric polytope", just like amplitudes of bi-adjoint scalar theory having the corresponding "associahedron" picture.

As an interesting generalization of bi-adjoint scalar theories, we would like to see how many good behaviors have been kept for these Lablled tree graphes. In this note we will show two nice observations. First, for complicated cubic tree Feynman diagrams produced by iterative procedure, we will show that they can be re-organized to some much simpler effective Feynman diagrams. As it will be seen, using these effective Feynman diagrams, it is much easier to capture the theory, since the pole structure will be much more organized and the connection to geometric picture (i.e., the combinatoric polytope) will be more transparent. The second observation is that for CHY-integrands coming from PT-factors, as shown in $[18,19]$ there is a procedure to select a subset of cubic Feynman diagrams with a particular pole structure by multiplying some cross ratio factors. This idea has been explored recently in the construction of one-loop CHY-integrands of bi-adjoint scalar theory in [20]. Since the Cayley tree's are the natural generalization of the PTfactors, we would like to ask if there is a similar procedure, such that we could pick out terms with a given pole structure. In this paper, we have suggested an algorithm to achieve this goal. Although we could not give a rigorous proof for the algorithm, ${ }^{3}$ we have checked many examples. We find that our algorithm works not only for Cayley tree's, but also other weight four CHY-integrands containing only simple poles.

We organize this paper as follows. In section 2, we have carefully analyzed the structure of effective Feynman diagrams, including two types of effective vertexes, and show how to read out effective Feynman diagrams from a given Cayley tree. From these compact effective Feynman diagrams, we show how to connect to the geometric picture. In section 3, by carefully analyzing different kinds of cross ratio factors coming from denominator and numerators, we show how to algorithmically construct their combinations to pick out particular pole from all cubic Feynman diagrams produced by any CHY-integrand having only simple poles. We illustrate the algorithm by various non-trivial examples. In the section 4 , a brief discussion of our work is presented.

\footnotetext{
${ }^{2}$ For the graph to be tree, given a pair of nodes $(i, j)$, there is at most one edge connecting them.

${ }^{3}$ For the case of PT-factors, such a proof is straightforward.
} 

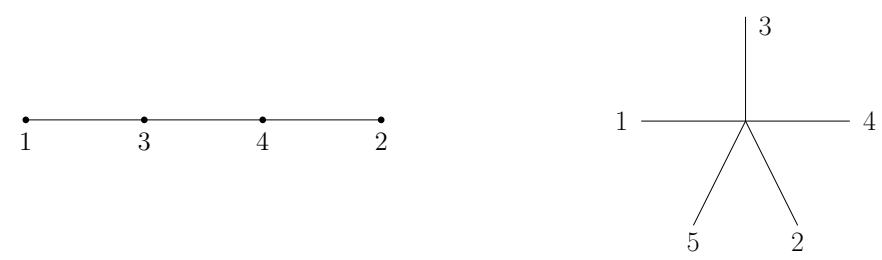

Figure 2. Cayley tree of PT $(1,3,4,2,5)$ and the corresponding effectively Feynman diagrams (vertex).

\section{The effective Feynman diagrams}

In this section, we will present our first main result, i.e., the effective Feynman diagrams. We will show how to read out effective vertexes from a given Cayley tree and how to glue these vertexes together to give an effective Feynman diagram. To understand the construction, we demonstrate with several examples. We discuss also the enumeration problem, i.e., how many real cubic Feynman diagrams are coded by a single effective Feynman diagram. Finally, using some examples, we show how to use the effective Feynman diagrams to understand the geometric picture, i.e., the polytope of the amplitudes.

\subsection{Two basic types of effective Feynman vertexes}

In [1] the iterative construction of cubic Feynman diagrams of CHY-integrands $\left(C_{n}\left(T_{n-1}\right)\right)^{2}$ with $C_{n}\left(T_{n-1}\right)$ defined by Cayley tree's has been given. ${ }^{4}$ It is observed that with complicated Cayley tree's, there are a lot of Feynman diagrams, thus a good way to organize these diagrams will be very useful for our further understanding of various questions related to these theories.

Among all Cayley tree's, there are two special types, for which the pole structures are clear. The first one is just a line, for example, the Cayley tree $T_{4}$ with edge-list $\{\{1,3\},\{3,4\},\{4,2\}\}$ (see the left graph in the figure 2). When rewriting into the $S L(2, C)$ covariant form, it is nothing but the familiar Parke-Taylor graph (or the "Hamiltonian graph"). Thus the left example given in the figure 2 is just $P T(\{1,3,4,2,5\})$. Using the algorithm in $[3,14,15]$, we could easily get the related cubic Feynman diagrams: they are all five point cubic Feynman diagrams respecting the ordering $\alpha$ (see the figure 3). Since we know the pole structure of these Feynman diagrams, we can compactly represent them by "an effective Feynman vertex" $V_{C}$, which is defined as (see the right graph in the figure 2)

$$
\begin{aligned}
V_{C}(\alpha) \equiv & \{\text { the sum of all color ordered cubic Feynman diagrams } \\
& \text { respecting the ordering } \alpha\}
\end{aligned}
$$

Another special example is the star graph, where among $(n-1)$-points, $(n-2)$ of them connect to the remaining point (see, for example, figure 4). Using the algorithm in [1], we

\footnotetext{
${ }^{4}$ Please remember that the Cayley tree is the gauge fixed version of covariant CHY-integrand, see (1.10) and (1.11).
} 



Figure 3. The expansion of the effective vertex $V_{C}(1,3,4,2, n=5)$ and the corresponding cubic Feynman diagrams.

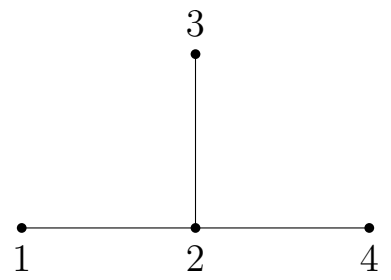

Figure 4. Star graph of $n=5$.

could get the related cubic Feynman diagrams with the permutation symmetry of $(n-2)$ points (see for example, figure 5 and 6 ). To understand the pattern, first let us relate an ordered sequence with a cubic Feynman diagram:

$$
\left(a_{1} ; a_{2}, a_{3}, \ldots, a_{n-1} ; a_{n}\right) \rightarrow \frac{1}{\prod_{t=2}^{n-2} s_{a_{1} a_{2} \ldots a_{t}}} .
$$

Using this notation, all Feynman diagrams coming from the star graph in the figure 4 can be summarized as $(1 ;\{2\} \amalg\{3\} \amalg\{4\} ; 5)$, which will be the sum of sequences of the form in (2.2) (see the figure 6). In this writing, we have defined the "shuffle" algebra. For a two ordered sets, their shuffle is defined as

$$
\begin{aligned}
& \alpha ш \emptyset=\alpha, \quad \emptyset ш \beta=\beta, \\
& \alpha ш \beta=\left\{\alpha_{1},\left\{\alpha_{2}, \ldots, \alpha_{m}\right\} \amalg \beta\right\}+\left\{\beta_{1}, \alpha \amalg\left\{\beta_{2}, \ldots, \beta_{k}\right\}\right\}
\end{aligned}
$$

where $\alpha_{1}, \beta_{1}$ are the first elements in the sets $\alpha, \beta$. Because we know very well the pattern for the star graph, we can compactly represent them by another "effective Feynman vertex" $V_{P}$, where the subscript $\mathrm{P}$ means the P-type vertex (the permutation type vertex). 

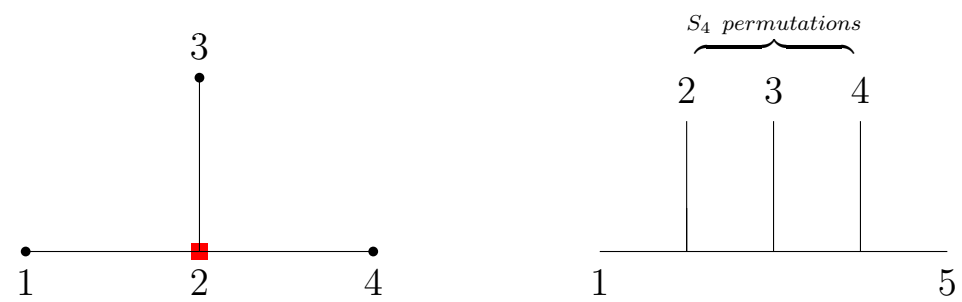

Figure 5. The 2-original Star graph and the corresponding effectively Feynman diagram (vertex).


Figure 6. The expansion of the effective vertex $V_{P}(1 ;\{2\} ш\{3\} \amalg\{4\} ; 5)$ and the corresponding comb-like Feynman diagrams.

For example, the start graph in the figure 4 can be rewritten as $V_{P}(2 ;\{1\} \amalg\{3\} \amalg\{4\} ; 5)$ (as shown in the figure 5 and 6$)$. The $V_{P}(2 ;\{1\} \amalg\{3\} \amalg\{4\} ; 5)$ vertex contains three parts: the starting external leg 2 , the ending external leg $n=5$ and the middle sequence coming from shuffle algebra.

The shuffle algebra $w$ is crucial for our compact representation of Feynman diagrams. Later we will meet more complicated shuffle algebras, such as

$$
\{2\} \varpi\{3\} \amalg\{4,5,6\}, \quad\{2\} \amalg\{3\} \amalg\{\{4\} \amalg\{5\}, 6\}
$$

The corresponding sequences produced by them should be easily worked out using (2.3).

Up to now we see that for above two special Cayley tree's, we have complete understanding of the pole structure of corresponding Feynman diagrams. These two special Cayley tree's correspond to the line and vertex (with multiple branches) structures in the general tree graphs respectively. Since every tree is constructed just by lines and vertexes with branches, it is very natural to guess that we should be able to compactly represent all Feynman diagrams coming from arbitrary Cayley tree by properly using about two special structures of Feynman diagrams. In the later subsections, we will use various examples 

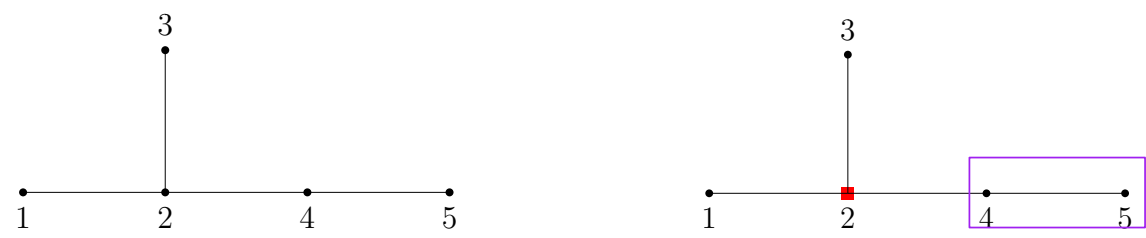

Figure 7. The Next-to-Star graph of $n=6$.
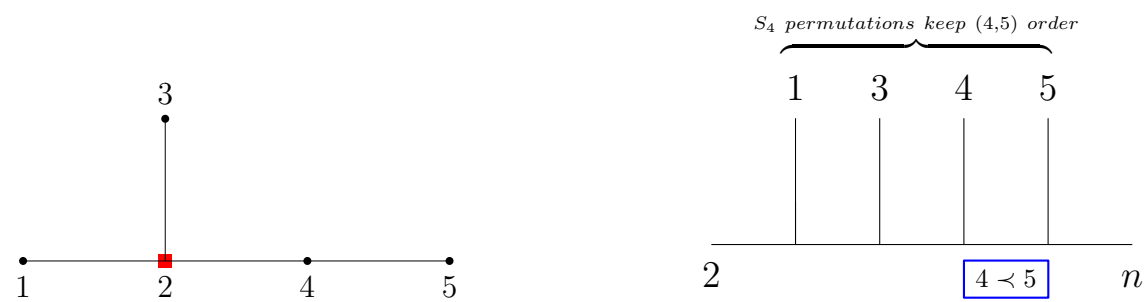

Figure 8. $V_{P}(2 ;\{1\} \amalg\{3\} \amalg\{4,5\} ; n=6)$ and the corresponding effective Feynman diagram.

to demonstrate the idea, especially the algorithmic way to read out all effective Feynman diagrams.

\subsection{The example of next-to-Star graph}

Starting from this subsection, we will show how to decompose an arbitrary Cayley tree to two basic structures (i.e., the line and the vertex) and then using the corresponding effective vertexes we could glue them together to give the corresponding effective Feynman diagrams. To get the idea, let us start with the simplest nontrivial example, i.e., the $n=6$ Next-to-Star graph as shown in the figure 7. This Cayley tree gives 18 cubic Feynman diagrams. To organize them, let us mark the node 2 first since it is the vertex with three branches in the Cayley tree (we marked it with a red square in the figure). Now we remove the point 2 and its adjacent edges, i.e., the edges $\{1,2\},\{3,2\},\{4,2\}$, and get three subgraphs. Among three subgraphs, two of them have no further structure, but one of them, does. For the nontrivial subgraph, we construct its substructures by considering all possible contracting of edges. The first case is that no edge has been contracted. Using the star graph of node 2 and its corresponding effective Feynman vertex, we get the effective diagram with only one effective vertex

$$
V_{P}(2 ;\{1\} \amalg\{3\} \amalg\{4,5\} ; n)
$$

When expanding the effective diagram, the corresponding cubic Feynman diagrams are given in the figure 8. It is worth to notice that different from the pure star graph, now the effective vertex contains the sub-structure, i.e., in the comb-like Feynman diagrams, the leg 4 is always near the leg 2 than the leg 5 by the shuffle algebra. To emphasize this important point, we have mark the $\{4,5\}$ order on the comb-like diagram as $4 \prec 5^{5}$ under

\footnotetext{
${ }^{5}$ In the original Cayley tree, the node 4 is closer to the marked point 2 than node 5 . In the following, we will use the left directed $\prec$ to represent the precursor and omit the order mark with the 2 adjacent points.
} 



Figure 9. Star graph and the line.
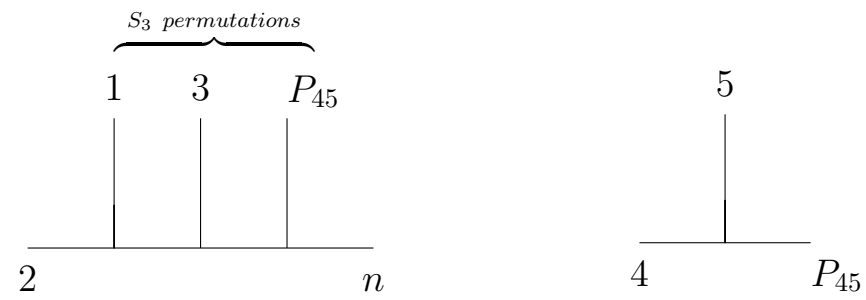

Figure 10. $V_{P}\left(2 ;\{1\} \varpi\{3\} \amalg\left\{P_{45}\right\} ; n=6\right)$ and $V_{C}\left(\left\{4,5, P_{45}\right\}\right)$.



Figure 11. The corresponding effective Feynman diagram.

the legs of 4,5 in the effective Feynman diagram. One can check that this effective vertex gives $\frac{4 !}{2 !}=12$ comb-like cubic Feynman diagrams.

The second case is the contraction of the edge $\{4,5\}$ to a node denoted by $P_{45}$. With this contraction, the Cayley tree is separated to two parts as shown in the figure 9. The left graph is the standard star graph while the right graph is the standard Hamiltonian graph. Thus as shown in the figure 10 by using our effective vertexes, the left graph gives $V_{P}\left(2 ;\{1\} \amalg\{3\} \amalg\left\{P_{45}\right\} ; n=6\right)$, which can be expanded to six cubic Feynman diagrams, while the right graph gives $V_{C}\left(\left\{4,5, P_{45}\right\}\right)$, which is just one cubic Feynman diagram. Using the propagator $P_{45}$ to glue these two effective vertexes together, we get an effective Feynman diagram as shown in the figure 11 with the following expression

$$
V_{P}\left(2 ;\{1\} \amalg\{3\} \amalg\left\{P_{45}\right\} ; n=6\right) \frac{1}{P_{45}^{2}} V_{C}\left(\left\{4,5, P_{45}\right\}\right)
$$

Since the left part gives 6 diagrams while the right part, just one, the effective Feynman diagram (2.6) gives six cubic Feynman diagrams. When we add 12 diagrams coming from (2.5), we do get total 18 diagrams as done using the iterative construction.

In above construction of effective Feynman diagrams, we have used the node 2 as the starting (marked) point. In general, we can choose any point to start with the whole construction. Let us redo this example with node 4 as the staring point as shown in the 




Figure 12. The node 4 as the marked point.


Figure 13. $V_{P}(4 ;\{5\} \varpi\{2,\{3 \amalg 1\}\} ; n)$ and the corresponding effective Feynman diagram.

figure 12. Again we need to consider the subgraphs: one is just a node 5 and another one, with nodes 1,2,3. For the later one, we consider various contractions as above. Now there are two edges, so we have following four types of contractions: (a) no contraction at all; (b) the edge $\{2,3\}$ has been contracted; (c) the edge $\{1,2\}$ has been contracted; (d) all edges $\{1,2\},\{2,3\}$ have been contracted. For the case (a), using the effective vertex of star graph of node 4 , we get the contribution (we have also given the counting when expanding the effective Feynman diagram)

$$
V_{P}(4 ;\{5\} ш\{2,\{3 \amalg 1\}\} ; n=6), \quad \#=\frac{4 !}{3}=8
$$

as shown in the figure 13. It is worth to notice the substructure in the shuffle algebra $\{2,\{3 \amalg 1\}\}$ to keep the orderings between $\{2,3\}$ and $\{2,1\}$ to the node 4 . This effective Feynman diagram contain $\frac{4 !}{3}=8$ cubic Feynman diagrams. For the case (b), (c) and (d) with contracted edge, we have separated the Cayley tree's to two parts: the first part is given in the figure 14 and the second part is given in the figure 15, where the corresponding effective Feynman vertexes have also been written down. Having known the two parts, we use the propagator to connect them and write down the final effective Feynman diagrams as shown in the figure 16

$$
\begin{array}{ll}
(b)=V_{P}\left(4 ;\{5\} \amalg\left\{P_{23}, 1\right\} ; n\right) \frac{1}{P_{23}^{2}} V_{C}\left(\left\{P_{23}, 2,3\right\}\right), & \#=\frac{3 !}{2}=3 \\
(c)=V_{P}\left(4 ;\{5\} \amalg\left\{P_{12}, 3\right\} ; n\right) \frac{1}{P_{12}^{2}} V_{C}\left(\left\{P_{12}, 1,2\right\}\right) & \#=\frac{3 !}{2}=3 \\
(d)=V_{P}\left(4 ;\{5\} \amalg\left\{P_{123}\right\} ; n\right) \frac{1}{P_{123}^{2}} V_{P}\left(2 ;\{1\} \amalg\{3\} ; P_{123}\right. & \#=2 \times 2=4
\end{array}
$$

It is worth to notice that for the case (d), the part in the figure 14 can be considered as the star graph of node 2 with infinity node $P_{123}$ or as the Hamiltonian graph with effective 

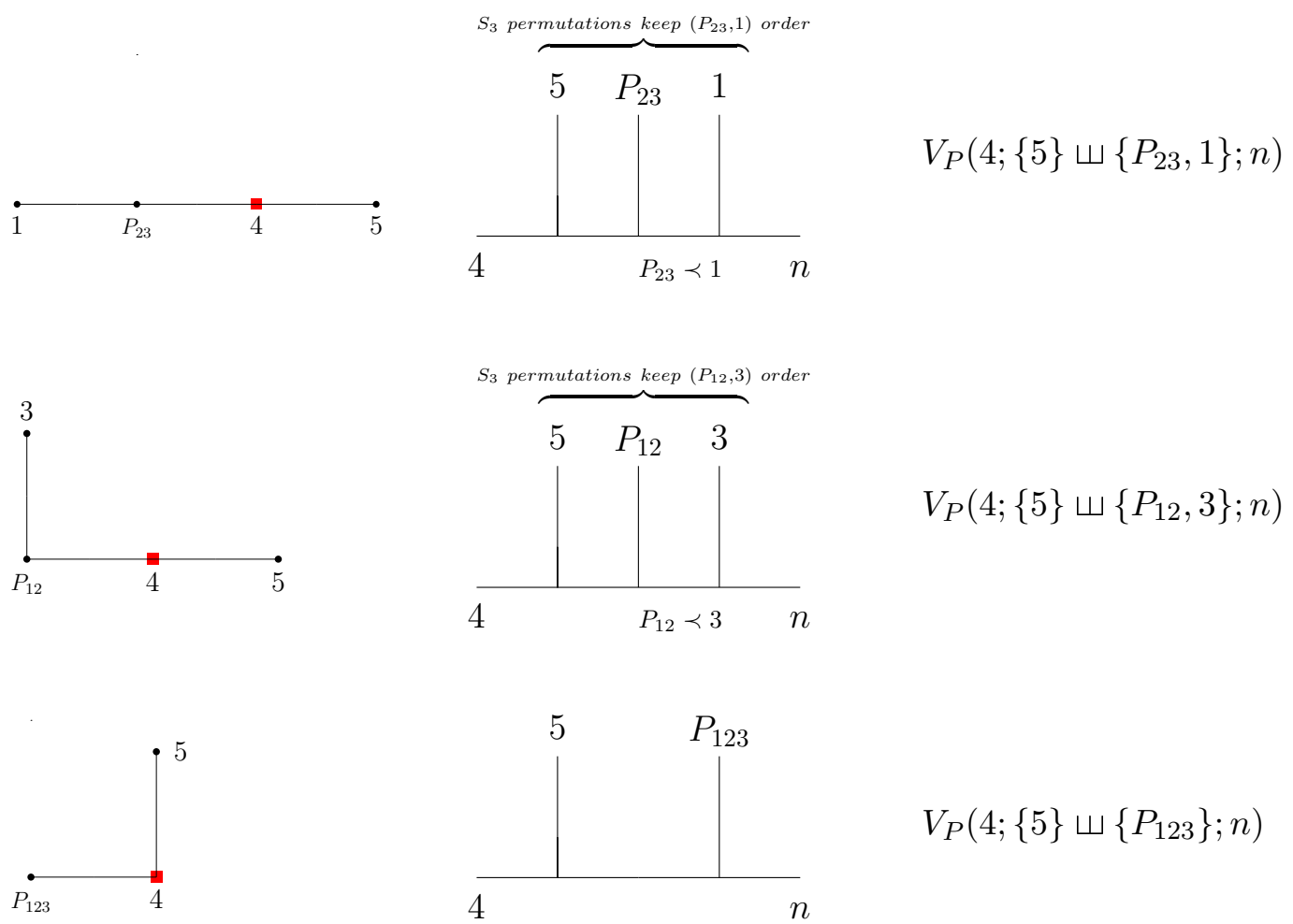

$V_{P}\left(4 ;\{5\} \amalg\left\{P_{123}\right\} ; n\right)$

Figure 14. The structure after contractions and the effective Feymann vertexes.
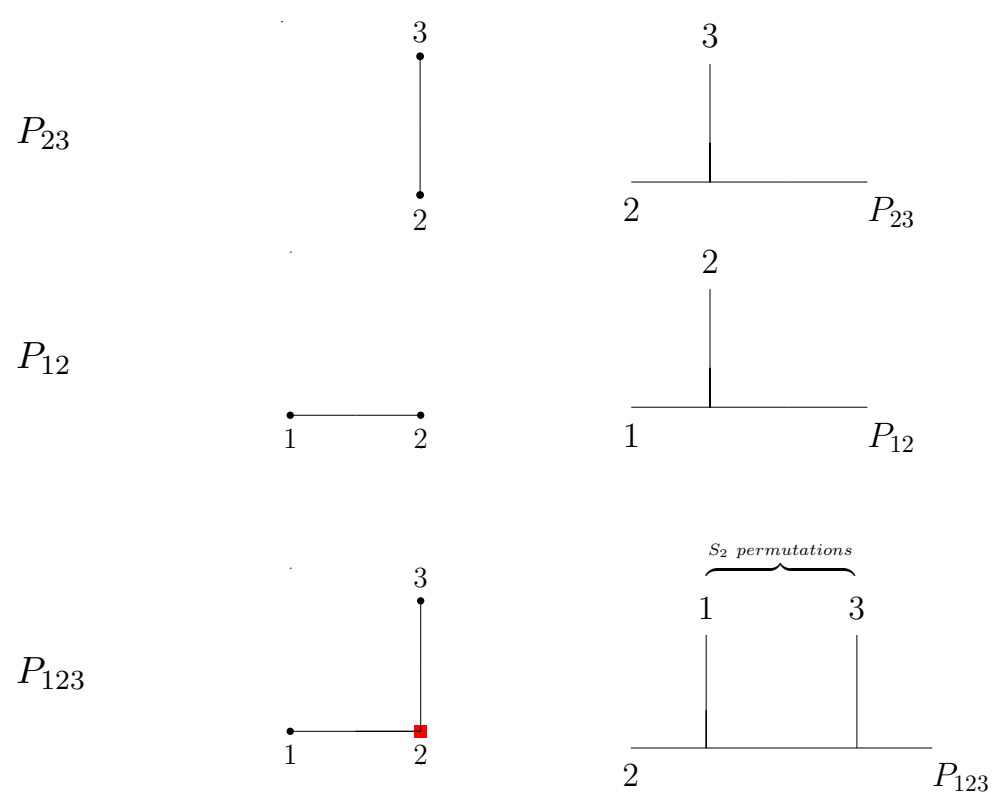

Figure 15. The contracted part the corresponding sub-Feymann diagrams. 

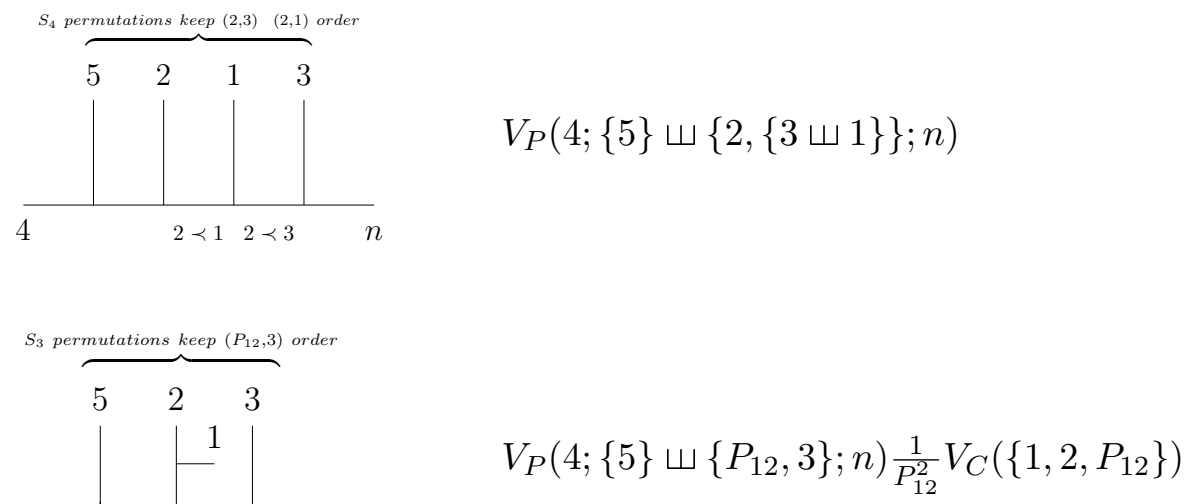

$$
V_{P}\left(4 ;\{5\} \amalg\left\{P_{12}, 3\right\} ; n\right) \frac{1}{P_{12}^{2}} V_{C}\left(\left\{1,2, P_{12}\right\}\right)
$$
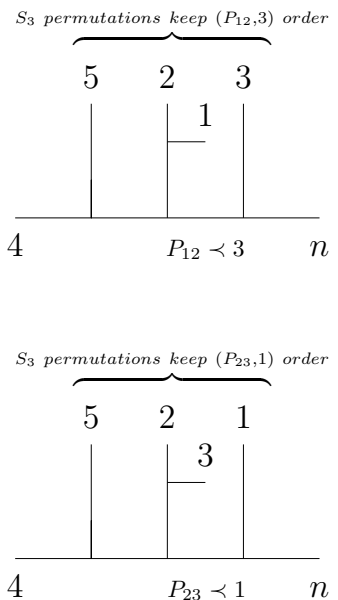

$$
V_{P}\left(4 ;\{5\} \amalg\left\{P_{23}, 1\right\} ; n\right) \frac{1}{P_{23}^{2}} V_{C}\left(\left\{2,3, P_{23}\right\}\right)
$$

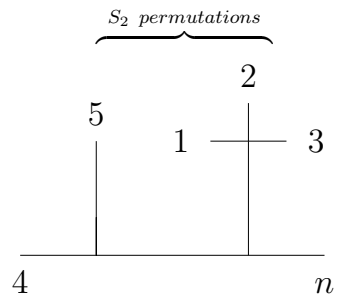

$$
V_{P}\left(4 ;\{5\} \amalg\left\{P_{123}\right\} ; n\right) \frac{1}{P_{123}^{2}} V_{C}\left(\left\{1,2,3, P_{123}\right\}\right)
$$

Figure 16. The full Effective Feynman diagram representation.

vertex $V_{C}\left(\left\{1,2,3, P_{123}\right\}\right)$. When we add all effective Feynman diagrams together, we do get 18 Feynman diagrams.

Having done above two calculations, now we can summarize the algorithm:

- (A) At the first step, we choose a marked node, for example, $k$.

- (B) Then we remove all edges connecting to the marked node $k$. Now the Cayley tree is separated to node $k$ and several subgraphs, which we can denote as $K_{1}, K_{2}, \ldots, K_{t}$.

- (C) For each subgraph $K_{i}$, we need to consider its all substructures by all possible contractions of edges in the subgraph. For a given contraction, we will generate following data: (1) First we shrink all contracted edges in the subgraph $K_{i}$ (so nodes at the two ends of the edge will be merged to a single node) to generate a new graph $\widetilde{K}_{i} ;(2)$ Secondly, all edges having been contracted will become several disconnected sub-Cayley tree, which we will denote as $K_{i, j}$. 
- (D) Having data from the step (C), now we have an effective vertex $V_{P}$, which is given by reconnecting the node $k$ with all $\widetilde{K}_{i}$. The roughly expression will be

$$
V_{P}\left(k ; \widetilde{K}_{1} ш \widetilde{K}_{2} ш \ldots ш \widetilde{K}_{t} ; n\right)
$$

where for each $\widetilde{K}_{i}$ in the shuffle algebra $(2.9)$, possible embedded structure will appear as such given in (2.7).

- (E) For each sub-Carley tree $K_{i, j}$, we can repeat the steps from (A) to (D) to get the corresponding effective vertex $V_{K_{i, j}}$. Then we connect vertex $V_{K_{i, j}}$ to the merged node in $\widetilde{K}_{i}$ by corresponding propagator to construct the effective Feynman diagram.

- (F) Iterating above steps, we will obtain all effective Feynman diagrams for any Cayley tree.

One should use above two calculations to get more clear picture about our algorithm. Some remarks for the algorithm are following:

- (a) Although in general we will get the $V_{P}$ vertex in above algorithm, when the subCayley tree is just a line (i.e., the Hamiltonian graph), we do not need to consider its various substructure by contractions. For this simple case, we just need to use the color-ordered effective vertex $V_{C}$ as explained below the equation (2.8).

- (b) Our algorithm is also recursive, but top-down style.

- (c) From above two calculations, we see that stating from different marked nodes, we can have different organizations, i.e., different sets of effective Feynman diagrams. Thus a good choice of the marked node is very important to get more compact expressions.

\subsection{Enumerate Feynman diagrams from an effective Feynman diagram}

Since we have used the concept of effective vertex, each effective Feynman diagram will code several cubic Feynman diagrams when expanding each effective vertex, thus the counting of these cubic Feynman diagrams is a very important check for our algorithm. In this subsection, we will consider this problem.

For an effective Feynman diagram, its counting $N_{F}$ is given by

$$
N_{F}=\prod_{i=1}^{t} n_{i}
$$

where $t$ is the number of effective vertexes in the diagram and for each effective vertex, $n_{i}$ is the number of its expansion to cubic Feynman sub-diagrams. Since effective vertex can only be two types, i.e., either the $P$-type or the $C$-type, we discuss them one by one.

For the $C$-type effective vertex, the $\left|V_{C}\left\{\left(l_{1}, l_{2}, \ldots, l_{n}\right)\right\}\right|$ enumerates all the cubic Feynman trees respecting the colour order of the list $\left\{\left(l_{1}, l_{2}, \ldots, l_{n}\right)\right\}$. According to [1], it is the $n$-th Catalan number $\mathrm{Cat}_{n}$, which is given directly in terms of binomial coefficients by

$$
\mathrm{Cat}_{n}=\frac{1}{n+1}\left(\begin{array}{c}
2 n \\
n
\end{array}\right)=\frac{(2 n) !}{(n+1) ! n !}=\prod_{k=2}^{n} \frac{n+k}{k} \quad \text { for } n \geq 0
$$




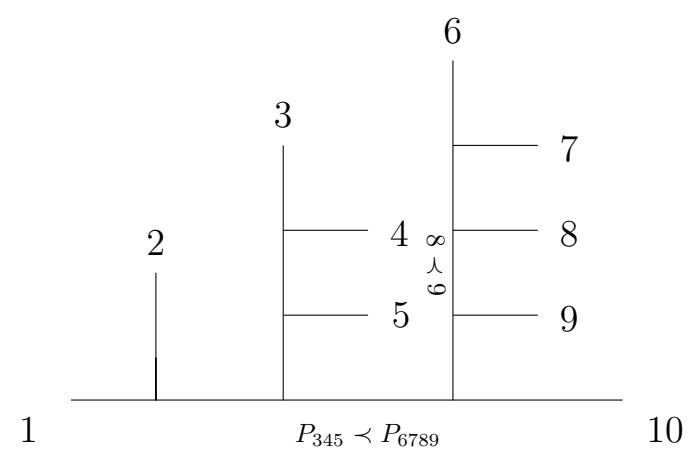

Figure 17. $V_{P}\left(1 ;\{2\} \amalg\left\{P_{345}, P_{6789}\right\} ; 10\right) \frac{1}{P_{345}} V_{P}\left(3 ;\{4\} \amalg\{5\} ; P_{345}\right) \frac{1}{P_{6789}} V_{P}\left(6 ;\{7\} \amalg\{8,9\} ; P_{6789}\right)$.

The first few Catalan numbers are given by

$$
\begin{aligned}
& \mathrm{Cat}_{2}=1 \text {, } \\
& \mathrm{Cat}_{3}=2 \text {, } \\
& \mathrm{Cat}_{4}=5 \text {, } \\
& \mathrm{Cat}_{5}=14 \text {, } \\
& \mathrm{Cat}_{6}=42 \\
& \mathrm{Cat}_{7}=132 \text {, } \\
& \mathrm{Cat}_{8}=429 \text {, } \\
& \text { Cat }_{9}=1430
\end{aligned}
$$

For the $P$-type vertex, the counting is not so trivial, since in general, the shuffle algebra contains various substructure. However, as we will see, the counting can be done level by level. Let us start with a typical situation, i.e., two arbitrary ordering list $\alpha=$ $\left\{\alpha_{1}, \alpha_{2}, \ldots, \alpha_{m}\right\}$ and $\beta=\left\{\beta_{1}, \beta_{2}, \ldots, \beta_{n}\right\}$. From (2.3), we see that the shuffle $\alpha \varpi \beta$ contains all possible permutations of the list $\alpha \cup \beta$, which preserve the relative ordering in $\alpha$ and $\beta$ respectively. Thus the counting is given by

$$
|\alpha \amalg \beta|=\frac{(m+n) !}{m ! n !},
$$

This counting can be easily generalized to multiple lists, thus we have

$$
\left|V_{P}\left(o ; \alpha_{1} \amalg \alpha_{2} ш \ldots ш \alpha_{k} ; n\right)\right|=\frac{\left(\sum_{i=1}^{k}\left|\alpha_{i}\right|\right) !}{\prod_{j=1}^{k}\left|\alpha_{j}\right| !}
$$

when all list $\alpha_{i}$ 's do not have substructure. When the $V_{P}$ has some substructures, we could recursively count the number. For example, for

$$
V_{P}(1 ;\{2,3 \amalg 4\} \amalg\{5,6 \amalg 7\} ; 8)
$$

there are two levels of shuffle algebra. For the first layer $\{2,3 \amalg 4\} \amalg\{5,6 \amalg 7\}$, using the formula (2.14), we get the counting $\frac{(3+3) !}{3 ! 3 !}=20$. For the second shuffle layer, i.e., $\{3 \varpi 4\}$ and $\{6 \sqcup 7\}$, each of them gives the number $\frac{(1+1) !}{1 ! 1 !}=2$. Putting them together, we get the counting $20 \times(2 \times 2)=80$.

Having known the counting of each effective vertex, we can get the counting for any effective Feynman diagram. For the effective Feynman diagram given in the figure 17, its expression is given by

$$
V_{P}\left(1 ;\{2\} \amalg\left\{P_{345}, P_{6789}\right\} ; 10\right) \frac{1}{P_{345}} V_{P}\left(3 ;\{4\} \amalg\{5\} ; P_{345}\right) \frac{1}{P_{6789}} V_{P}\left(6 ;\{7\} \amalg\{8,9\} ; P_{6789}\right)
$$




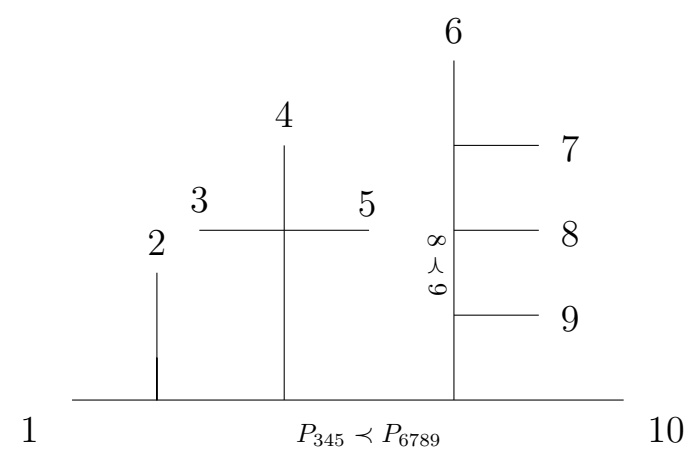

Figure 18. $V_{P}\left(1 ;\{2\} \amalg\left\{P_{345}, P_{6789}\right\} ; 10\right) \frac{1}{P_{345}} V_{C}\left(\left\{3,4,5, P_{345}\right\}\right) \frac{1}{P_{6789}} V_{P}\left(6 ;\{7\} ш\{8,9\} ; P_{6789}\right)$.



Figure 19. $C_{8}\{\{1,2\},\{1,3\},\{1,4\},\{2,5\},\{5,6\},\{5,7\}\}$.

thus the counting is given by

$$
\begin{aligned}
& \left|V_{P}\left(1 ;\{2\} \amalg\left\{P_{345}, P_{6789}\right\} ; 10\right)\right| \times\left|V_{P}\left(3 ;\{4\} \amalg\{5\} ; P_{345}\right)\right| \times\left|V_{P}\left(6 ;\{7\} \amalg\{8,9\} ; P_{6789}\right)\right| \\
= & \frac{(1+2) !}{2} \times \frac{(1+1) !}{1} \times \frac{(1+2) !}{2}=18
\end{aligned}
$$

For the example with mixing $V_{C}$ and $V_{P}$ types in the figure 18, its expression is given by

$$
V_{P}\left(1 ;\{2\} ш\left\{P_{345}, P_{6789}\right\} ; 10\right) \frac{1}{P_{345}} V_{C}\left(\left\{3,4,5, P_{345}\right\}\right) \frac{1}{P_{6789}} V_{P}\left(6 ;\{7\} \amalg\{8,9\} ; P_{6789}(2.17)\right.
$$

and the counting is given by

$$
\frac{(1+2) !}{2} \times \frac{1}{2+1}\left(\begin{array}{l}
4 \\
2
\end{array}\right) \times \frac{(1+2) !}{2}=18
$$

\subsection{Another example}

Having presented the general algorithm for the construction of effective Feynman diagrams for arbitrary Cayley tree's, in this subsection, we give another example for the Cayley tree in the figure 19:

If we pick the node 1 to be the marked point, the subgraphs related to it will be following three: $\{3\},\{4\},\{2,5,6,7\}$. Only the last one need to consider various contractions. There are three edges $\{\{2,5\},\{5,6\},\{5,7\}\}$ in this subtree, thus there are $2^{3}=8$ different contractions. ${ }^{6}$ After contraction and gluing back to node 1, we get these eight graphes in the figure 20. The corresponding effective $V_{P}$ vertex (2.9) can be found in the figure 23.

\footnotetext{
${ }^{6}$ The contraction of each edge is independent of each other. Thus for the marked point $o$, there are $2^{m}$ different contractions with $m=n-2-v_{o}$ where $v_{0}$ is the number of edges connecting to the marked point $o$.
} 
0 - contraction:

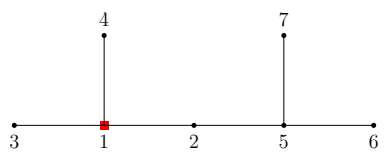

1 - contraction:
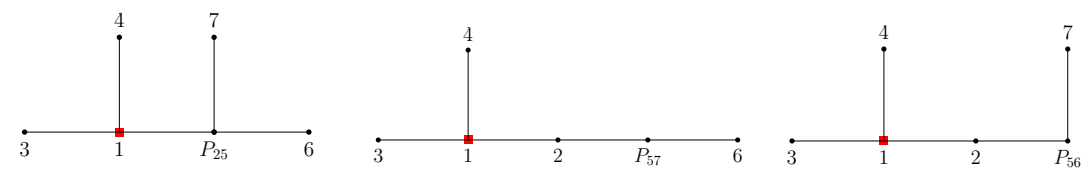

2 - contraction:
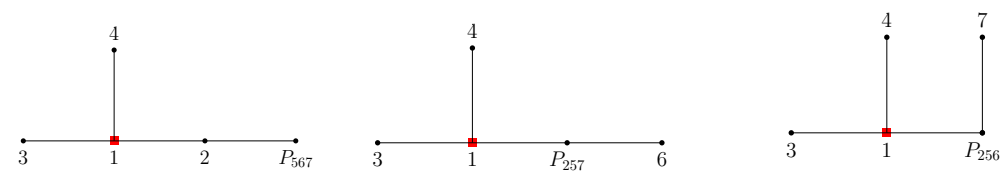

3 - contraction:

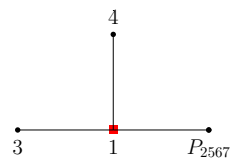

Figure 20. The sub-Cayley tree's with various contractions.

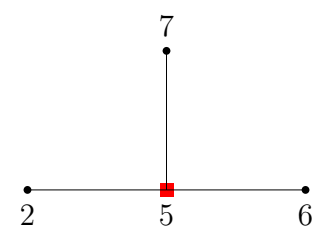

Figure 21. The last contraction $P_{2567}$.

For seven cases with nontrivial contractions in the figure 20, six of them are just the Hamiltonian lines, so the corresponding effective vertexes are just the $V_{C}$ types

$$
\begin{array}{lll}
V_{C}\left(\left\{P_{25}, 2,5\right\}\right), & V_{C}\left(\left\{P_{57}, 5,7\right\}\right), & V_{C}\left(\left\{P_{56}, 5,6\right\}\right), \\
V_{C}\left(\left\{P_{567}, 7,5,6\right\}\right), & V_{C}\left(\left\{P_{257}, 2,5,7\right\}\right), & V_{C}\left(\left\{P_{256}, 2,5,6\right\}\right)
\end{array}
$$

The $V_{C}$-type vertexes in the second line of above expression can also written as the $V_{P}$-type vertexes as given in the figure 23. The last contraction gives a star graph in the figure 21. Thus the corresponding effective vertex is $V_{P}\left(5 ; 2 \amalg 6 \amalg 7 ; P_{2567}\right)$. After putting these two effective vertexes together, we get the effective Feynman diagrams shown in the figure 22 . The corresponding algebraic expression and the counting for each effective Feynman is given in the figure 23. The overall counting for the Cayley tree is 248, which is consistent with the results obtained by recursion or integration rule in $[1,14,15]$.

Having discussed the effective Feynman diagrams starting from the node 1, we can do similar calculations for other nodes. For our current example, because the symmetry of 
0 - contraction:

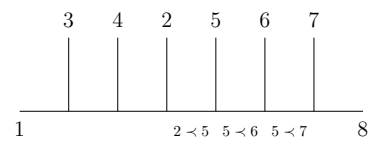

$1-$ contraction:
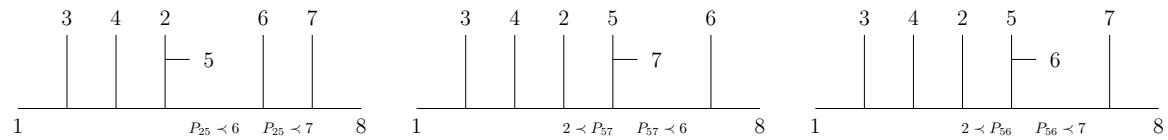

$2-$ contraction:
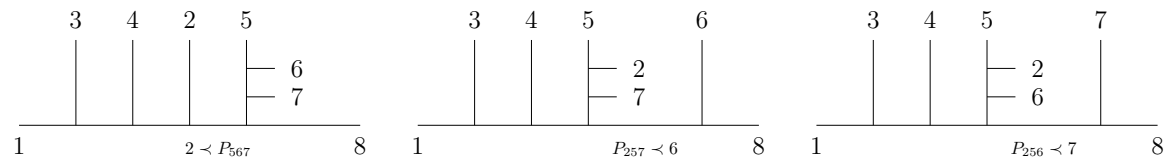

3 - contraction:

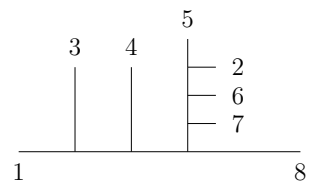

Figure 22. The full Effective Feynman diagram representation.

\begin{tabular}{|c|c|}
\hline effective Feynman diagram & counting \\
\hline$V_{P}(1 ; 3 ш 4 \amalg\{2,\{5,6 \amalg 7\}\} ; 8)$ & $\frac{(1+1+4) !}{1 ! 1 ! 4 !} \times \frac{(1+1) !}{1 ! 1 !}=60$ \\
\hline$V_{P}\left(1 ; 3 ш 4 \amalg\left\{P_{25}, 6 \amalg 7\right\} ; 8\right) \frac{1}{P_{25}} V_{C}(2,5)$ & $\frac{(1+1+3) !}{1 ! 1 ! 3 !} \times \frac{(1+1) !}{1 ! 1 !}=40$ \\
\hline$V_{P}\left(1 ; 3 ш 4 \amalg\left\{2,\left\{P_{57}, 6\right\}\right\} ; 8\right) \frac{1}{P_{57}} V_{C}(5,7)$ & $\frac{(1+1+3) !}{1 ! 1 ! 3 !}=20$ \\
\hline$V_{P}\left(1 ; 3 ш 4 \amalg\left\{2,\left\{P_{56}, 7\right\}\right\} ; 8\right) \frac{1}{P_{56}} V_{C}(5,6)$ & $\frac{(1+1+3) !}{1 ! 1 ! 3 !}=20$ \\
\hline$V_{P}\left(1 ; 3 \amalg 4 \amalg\left\{2, P_{567}\right\} ; 8\right) \frac{1}{P_{567}} V_{P}\left(5 ; 6 \amalg 7 ; P_{567}\right)$ & $\frac{(1+1+2) !}{1 ! 1 ! 2 !} \times \frac{(1+1) !}{1 ! 1 !}=24$ \\
\hline$V_{P}\left(1 ; 3 \amalg 4 \amalg\left\{P_{257}, 6\right\} ; 8\right) \frac{1}{P_{257}} V_{P}\left(5 ; 2 \amalg 7 ; P_{257}\right)$ & $\frac{(1+1+2) !}{1 ! 1 ! 2 !} \times \frac{(1+1) !}{1 ! 1 !}=24$ \\
\hline$V_{P}\left(1 ; 3 \amalg 4 \amalg\left\{P_{256}, 7\right\} ; 8\right) \frac{1}{P_{256}} V_{P}\left(5 ; 2 \amalg 6 ; P_{256}\right)$ & $\frac{(1+1+2) !}{1 ! 1 ! 2 !} \times \frac{(1+1) !}{1 ! 1 !}=24$ \\
\hline$V_{P}\left(1 ; 3 \amalg 4 \amalg P_{2567} ; 8\right) \frac{1}{P_{2567}} V_{P}\left(5 ; 2\right.$ Ш 6 Ш $\left.7 P_{2567}\right)$ & $\frac{(1+1+1) !}{1 ! 1 ! 1 !} \times \frac{(1+1+1) !}{1 ! 1 ! 1 !}=36$ \\
\hline
\end{tabular}

Figure 23. Effective Feymann diagrams and their enumaration.

Cayley tree, all nodes can be divided into three categories:

$$
\{2\} \quad\{1,5\} \quad\{3,4,6,7\}
$$

The second category has been done and now we move to another two categories. For the first category (i.e., node 2 as the starting point), there are $2^{6-2}=16$ contractions. Each contraction gives only one effective Feynman diagram. Thus we have following four group 
of diagrams. The first group is

$$
\begin{array}{ll}
V_{P}(2 ;\{1,3 ш 4\} \amalg\{5,6 \varpi 7\} ; 8), & \#=\frac{(3+3) !}{3 ! 3 !} \times 2 \times 2=80 \\
V_{P}\left(2 ;\{1,3 \amalg 4\} \amalg\left\{P_{56}, 7\right\} ; 8\right) \frac{1}{P_{56}^{2}} V_{C}\left(\left\{P_{56}, 5,6\right\}\right), & \#=\frac{(3+2) !}{3 ! 2 !} \times 2=20 \\
V_{P}\left(2 ;\{1,3 \amalg 4\} \amalg\left\{P_{57}, 6\right\} ; 8\right) \frac{1}{P_{57}^{2}} V_{C}\left(\left\{P_{57}, 5,7\right\}\right), & \#=\frac{(3+2) !}{3 ! 2 !} \times 2=20 \\
V_{P}\left(2 ;\{1,3 \amalg 4\} \amalg P_{567} ; 8\right) \frac{1}{P_{567}^{2}} V_{C}\left(\left\{P_{567}, 7,5,6\right\}\right), & \#=\frac{(3+1) !}{3 !} \times 2 \times 2=16
\end{array}
$$

The second group is

$$
\begin{array}{ll}
V_{P}\left(2 ;\left\{P_{13} \amalg 4\right\} \amalg\{5,6 \varpi 7\} ; 8\right) \frac{1}{P_{13}^{2}} V_{C}\left(\left\{P_{13}, 1,3\right\}\right), & \#=\frac{(2+3) !}{2 ! 3 !} \times 2=20 \\
V_{P}\left(2 ;\left\{P_{13} \amalg 4\right\} \amalg\left\{P_{56}, 7\right\} ; 8\right) \frac{1}{P_{13}^{2}} V_{C}\left(\left\{P_{13}, 1,3\right\}\right) \frac{1}{P_{56}^{2}} V_{C}\left(\left\{P_{56}, 5,6\right\}\right), & \#=\frac{(2+2) !}{2 ! 2 !}=6 \\
V_{P}\left(2 ;\left\{P_{13} \amalg 4\right\} \amalg\left\{P_{57}, 6\right\} ; 8\right) \frac{1}{P_{13}^{2}} V_{C}\left(\left\{P_{13}, 1,3\right\}\right) \frac{1}{P_{57}^{2}} V_{C}\left(\left\{P_{57}, 5,7\right\}\right), & \#=\frac{(2+2) !}{2 ! 2 !}=6 \\
V_{P}\left(2 ;\left\{P_{13} \amalg 4\right\} \amalg P_{567} ; 8\right) \frac{1}{P_{13}^{2}} V_{C}\left(\left\{P_{13}, 1,3\right\}\right) \frac{1}{P_{567}^{2}} V_{C}\left(\left\{P_{567}, 7,5,6\right\}\right), & \#=\frac{(2+1) !}{2 !} \times 2=6
\end{array}
$$

The third group is

$$
\begin{array}{ll}
V_{P}\left(2 ;\left\{P_{14} \amalg 3\right\} \amalg\{5,6 \varpi 7\} ; 8\right) \frac{1}{P_{14}^{2}} V_{C}\left(\left\{P_{14}, 1,4\right\}\right), & \#=\frac{(2+3) !}{2 ! 3 !} \times 2=20 \\
V_{P}\left(2 ;\left\{P_{14} \amalg 3\right\} \amalg\left\{P_{56}, 7\right\} ; 8\right) \frac{1}{P_{14}^{2}} V_{C}\left(\left\{P_{14}, 1,4\right\}\right) \frac{1}{P_{56}^{2}} V_{C}\left(\left\{P_{56}, 5,6\right\}\right), & \#=\frac{(2+2) !}{2 ! 2 !}=6 \\
V_{P}\left(2 ;\left\{P_{14} \amalg 3\right\} \amalg\left\{P_{57}, 6\right\} ; 8\right) \frac{1}{P_{14}^{2}} V_{C}\left(\left\{P_{14}, 1,4\right\}\right) \frac{1}{P_{57}^{2}} V_{C}\left(\left\{P_{57}, 5,7\right\}\right), & \#=\frac{(2+2) !}{2 ! 2 !}=6 \\
V_{P}\left(2 ;\left\{P_{14} \amalg 3\right\} \amalg P_{567} ; 8\right) \frac{1}{P_{14}^{2}} V_{C}\left(\left\{P_{14}, 1,4\right\}\right) \frac{1}{P_{567}^{2}} V_{C}\left(\left\{P_{567}, 7,5,6\right\}\right), & \#=\frac{(2+1) !}{2 !} \times 2=6
\end{array}
$$

The fourth group is

$$
\begin{array}{ll}
V_{P}\left(2 ; P_{134} \amalg\{5,6 \amalg 7\} ; 8\right) \frac{1}{P_{134}^{2}} V_{C}\left(\left\{P_{134}, 3,1,4\right\}\right), & \#=\frac{(1+3) !}{3 !} \times 2 \times 2=16 \\
V_{P}\left(2 ; P_{134} \amalg\left\{P_{56}, 7\right\} ; 8\right) \frac{1}{P_{134}^{2}} V_{C}\left(\left\{P_{134}, 3,1,4\right\}\right) \frac{1}{P_{56}^{2}} V_{C}\left(\left\{P_{56}, 5,6\right\}\right), & \#=\frac{(1+2) !}{2 !} \times 2=6 \\
V_{P}\left(2 ; P_{134} \amalg\left\{P_{57}, 6\right\} ; 8\right) \frac{1}{P_{134}^{2}} V_{C}\left(\left\{P_{134}, 3,1,4\right\}\right) \frac{1}{P_{57}^{2}} V_{C}\left(\left\{P_{57}, 5,7\right\}\right), & \#=\frac{(1+2) !}{2 !} \times 2=6 \\
V_{P}\left(2 ; P_{134} \amalg P_{567} ; 8\right) \frac{1}{P_{134}^{2}} V_{C}\left(\left\{P_{134}, 3,1,4\right\}\right) \frac{1}{P_{567}^{2}} V_{C}\left(\left\{P_{567}, 7,5,6\right\}\right), & \#=(1+1) ! \times 2 \times 2=8
\end{array}
$$

Adding them up, we get total 248 cubic Feynman diagrams.

For the third category, for example, the staring node 3 , the situation is a bit more complicated. There are $2^{5}=32$ contractions. But each contraction may give more than one effective Feynman diagrams, for example, the contractions of $P_{124567}$ and $P_{12567}$. If we choose the maximal valency node as the marked point at each recursive step of the 




Figure 24. $C_{10}\{\{1,2\},\{1,3\},\{1,4\},\{1,5\},\{3,7\},\{2,6\},\{4,8\},\{5,9\}\}$.

subgraph, we would eventually conclude that at least 41 effective Feynman Diagrams are needed to represent all Feynman diagram in the third category.

The above discussion tells us that to get more compact effective Feynman diagram representation, we should follow two principles throughout the whole recursive process:

- (1) At each step, select the maximal valency vertex as the starting point.

- (2) If there are multiple maximal valency vertexes, choose the one that the remaining subgraph have more disconnected branches after removing it and its adjacent edges.

The first one guarantees that there are minimal contracted sub-Cayley trees at each step. The second principle could reduce the number of Feynman subtrees for each effective vertex.

\subsection{More examples}

In this subsection, we give more examples to show how the effective Feynman diagrams can very compactly encode all cubic Feynman diagrams coming from a given Cayley tree, especially this Cayley tree has symmetric structure. Let us start from a $n=10$ Cayley tree given in the figure 24. It gives 6384 cubic Feynman diagrams, which is hard to write all of them down and get the picture of pole structures. However, with the symmetric structure, if we choose the node 1 as the starting point, we can see that all $2^{4}=16$ possible contractions give only 16 effective Feynman diagrams. Furthermore, these effective Feynman diagrams can be divided into five types: (1) no contraciton; (1) one contraction; (3) two contractions; (4) three contractions (5) four contractions. The typical effective Feynman diagrams and their counting have been given in the figure 25 .

Now let us consider another Cayley tree, which is given in the figure 26. Choosing the node 1 as the staring marked point, we have $2^{6}=64$ contractions and 32 effective Feynman diagrams. ${ }^{7}$ These 32 diagrams can be divided into ten types: (1) one without any contraction; (2) six of one contraction; (3) twelve of two contractions at the different

\footnotetext{
${ }^{7}$ When using the $V_{C}$ instead of $V_{P}$ for line structure, we can reduce the number of effective Feynman diagrams.
} 


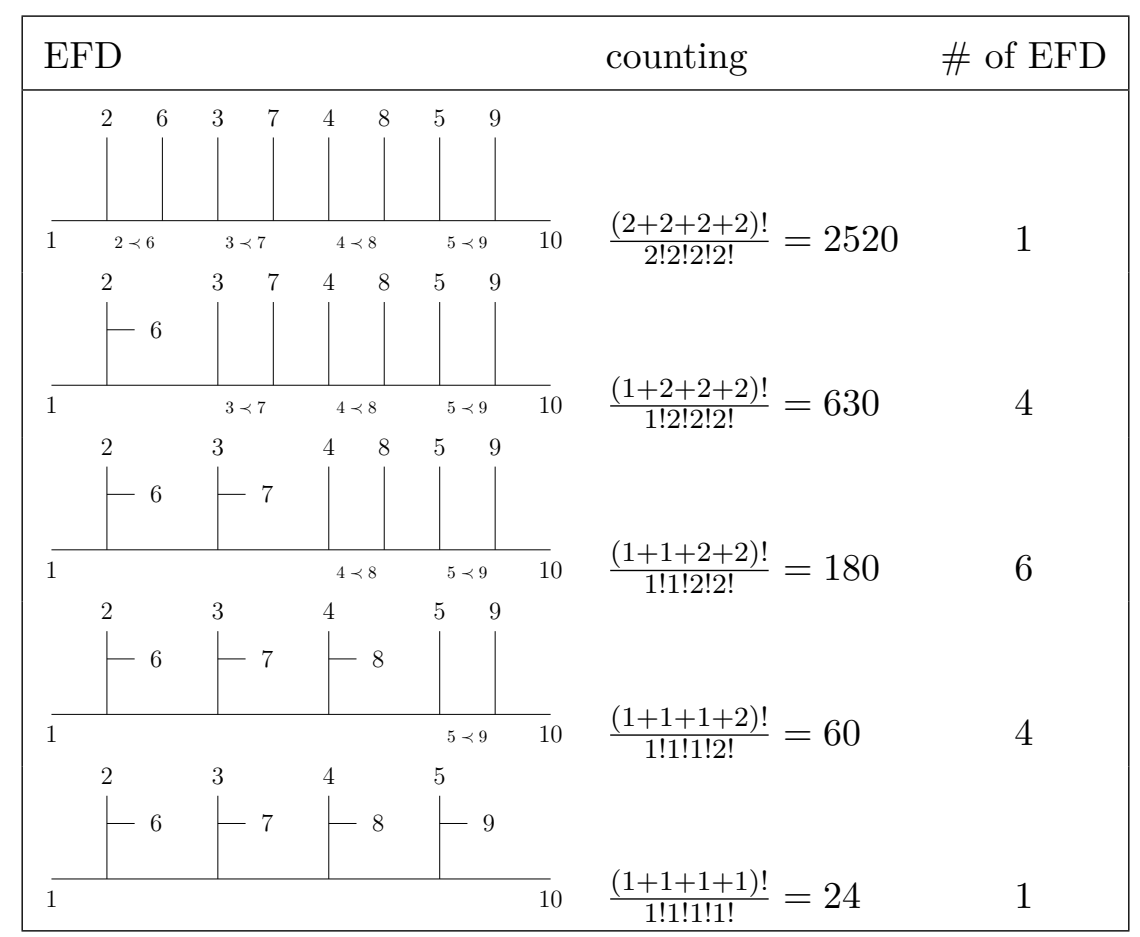

Figure 25. Enumerate the Effective Feymann diagrams.



Figure 26. $C_{11}\{\{1,2\},\{1,3\},\{1,4\},\{3,7\},\{3,8\},\{2,5\},\{2,6\},\{4,9\},\{4,10\}\}$.

branches; (4) three of two contractions at the same branch; (5) twelve of three contractions at the two different branches; (6) eight of three contractions at the three different branches; (7) twelve of four contractions at the three different branches; (8) three of four contractions at the two different branches; (9) six of five contractions at the three different branches; (10) one of six contractions. The typical effective Feynman diagrams and their counting are given in the figure 27. These effective diagrams have coded 40416 cubic Feynman diagrams. 


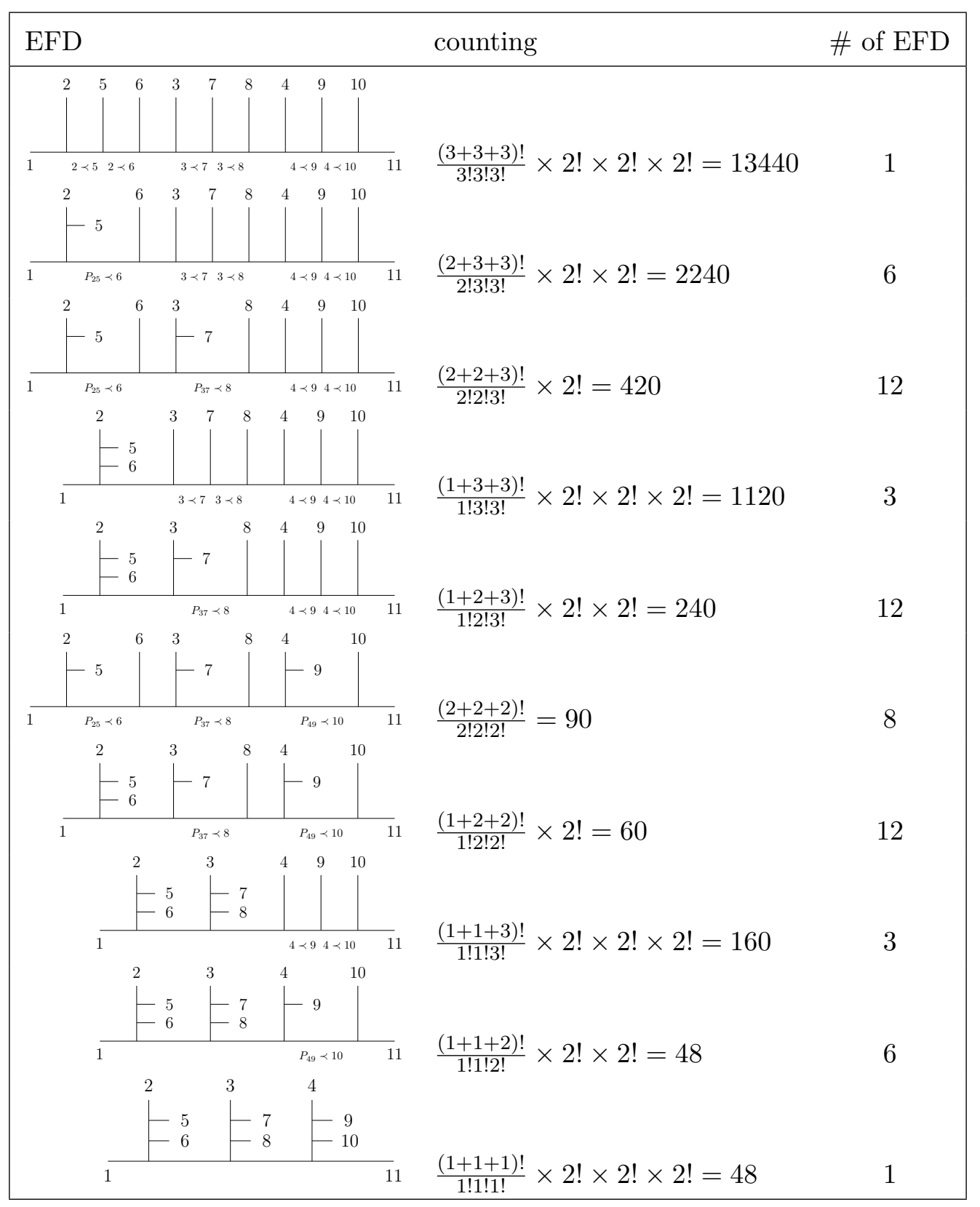

Figure 27. Enumerate the Effective Feymann diagrams.

\subsection{Application of effective Feynman diagrams}

In previous subsections, we have shown how to use the picture of effective Feynman diagrams to compactly code pole structures of all cubic Feynman diagrams coming from a given Cayley tree. In this subsection, we will show some applications of the new picture.

The first application is to a geometric object, the so called "Polytope of Feynman diagrams", which is defined for a collection of cubic Feynman diagrams of $n$ points as following

- (1) Each vertex of this polytope corresponds to a cubic Feynman diagrams (so there are $(n-3)$ poles). 

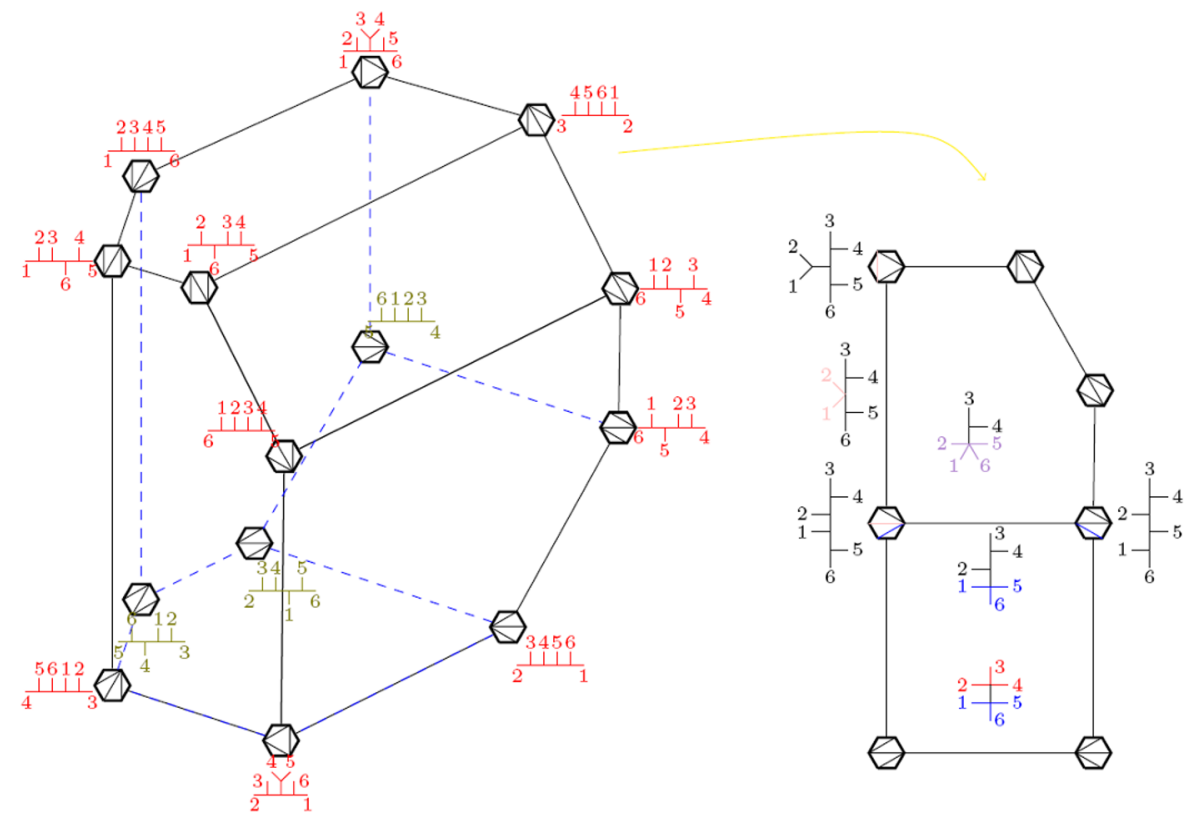

Figure 28. Associahedron of $(\mathrm{PT}(\{1,2,3,4,5,6\}))^{2}$.

- (2) Two vertexes will be connected by an edge when and only when they share same $(n-4)$ poles.

- (3) All vertexes on a surface share same $(n-5)$ poles.

- (4) In general, vertexes of a dimension $r$ surface share same $(n-3-r)$ poles.

Above construction of polytope has used the bottom-up approach. Our definition of effective vertexes has used an opposite approach, i.e., the top-down method. For example, for the CHY-integrand $(\operatorname{PT}(\{1,2, \ldots, n\}))^{2}$, all cubic Feynman diagrams are represented by a single effective vertex $V_{C}(\{1,2, \ldots, n\})$. This single vertex corresponds the $(n-3)$ dimension polytope, the so called "associahedron".

Its codimension one boundary correspond to fix a given pole, which corresponds to split the single $V_{C}$-type effective vertex to two $V_{C}$-type effective vertexes connected by this given pole, i.e,

$$
\begin{aligned}
& V_{C}(\{1,2,3,4,5,6\}) \\
\rightarrow & \begin{cases}V_{C}\left(\left\{1,2, P_{12}\right\}\right) \frac{1}{s_{12}} V_{C}\left(\left\{P_{12}, 3,4,5,6\right\}\right) & 6 \text { cases }: s_{12}, s_{23}, s_{34}, s_{45}, S_{56}, s_{61} \\
V_{C}\left(\left\{1,2,3, P_{123}\right\}\right) \frac{1}{s_{123}} V_{C}\left(\left\{P_{123}, 4,5,6\right\}\right) & 3 \text { cases }: s_{123}, s_{234}, s_{345}\end{cases}
\end{aligned}
$$

Thus there are 9 faces. By counting each effective Feynman diagram in (2.25), we see that 6 faces have five edges and five vertexes while 3 faces have four edges and four vertexes. We can split effective vertex further to get the representation of edges from results in $(2.25){ }^{8}$

\footnotetext{
${ }^{8}$ Such a picture has been discussed in [18].
} 


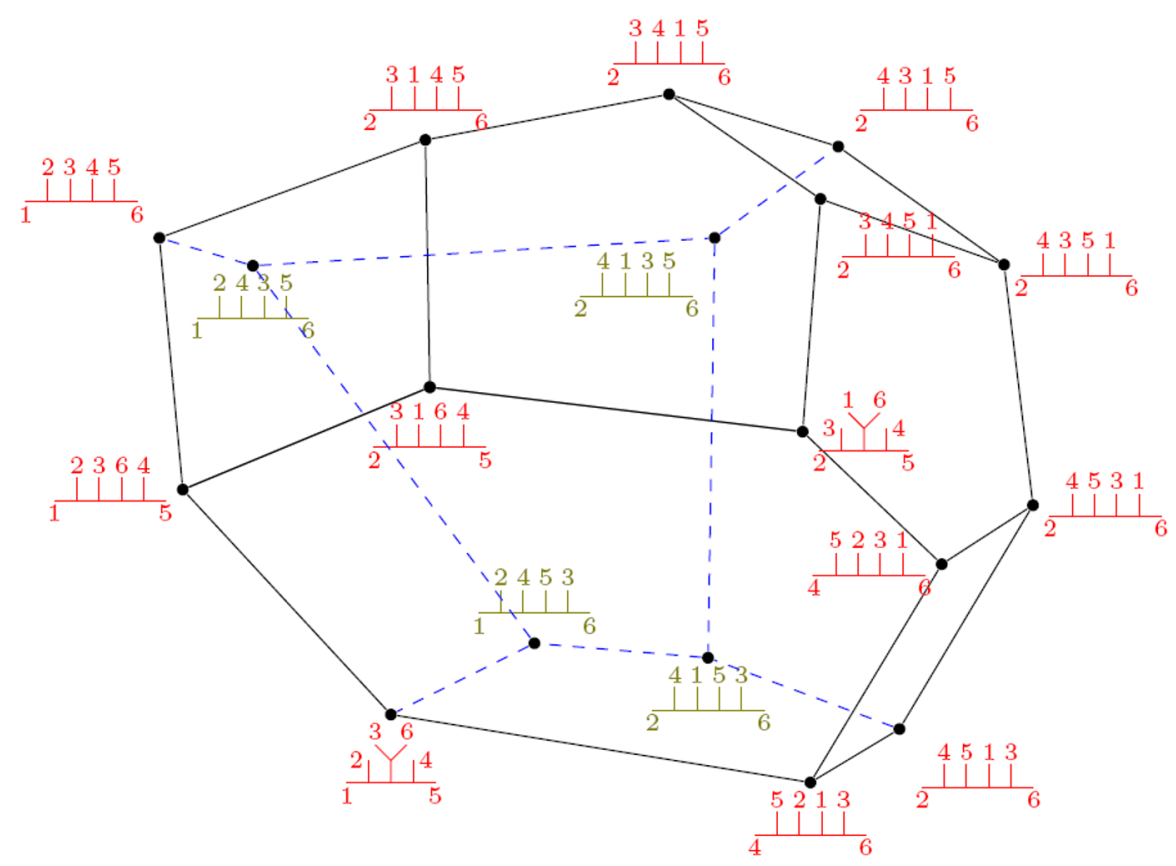

Figure 29. The polytope of the Next-to-Star graph with $n=6$.

The same splitting picture holds for the $V_{P}$-type effective vertex. For the Cayley tree given in the figure 8, the whole polytope is given by two effective Feynman diagrams:

$$
\begin{aligned}
& F_{A}=V_{P}\left(2 ;\{1\} \uplus\{3\} \uplus\left\{P_{45}\right\} ; n\right) \frac{1}{P_{45}^{2}} V_{C}\left(\left\{4,5, P_{45}\right\}\right) \\
& F_{B}=V_{P}(2 ;\{1\} \uplus\{3\} \uplus\{4,5\} ; n)
\end{aligned}
$$

Since the effective diagram $F_{A}$ has a fixed pole $s_{45}$, it defines a two-dimension surface instead of three-dimension volumn. For the effective diagram $F_{B}$, when considering the relative orderings we have following types of splitting

$$
\begin{array}{cl}
F_{B}=V_{P}(2 ;\{1\} ш\{3\} \amalg\{4,5\} ; n) \\
\rightarrow \begin{cases}V_{P}\left(2 ;\{1\} ; P_{12}\right) \frac{1}{s_{12}} V_{P}\left(P_{12} ;\{3\} ш\{4,5\} ; n\right\} & 3 \text { cases }: s_{21}, s_{23}, s_{24} \\
V_{P}\left(2 ;\{1\} \amalg\{3\} ; P_{123}\right) \frac{1}{s_{123}} V_{P}\left(P_{123} ;\{4,5\} ; n\right\} & 4 \text { cases }: s_{213}, s_{214}, s_{234}, s_{245} \\
V_{P}\left(2 ;\{1\} \amalg\{3\} \amalg\{4\} ; P_{1234}\right) \frac{1}{s_{1234}} V_{P}\left(P_{1234} ;\{5\} ; n\right) & 3 \text { cases }: s_{2134}, s_{2145}, s_{2345}\end{cases}
\end{array}
$$

Adding together, we find the polytope has 11 two-dimension surfaces. Each surface is defined by an effective Feynman diagram. Counting the effective Feynman diagrams, we can find that there are 4 surfaces with four edges, 5 surfaces with five edges and 2 surfaces with six edges. Which two surfaces share an edge can also be easily identified.

The second application is for CHY-integrands given by the multiplication of two different Cayley functions. Again we can draw the effective Feynman diagrams of these two Cayley functions to find the common sub-diagrams. Let us consider one example, i.e., two 
star graphes of $n=6$, one with the 2 at the center and another one, 4 in the center. To find the common sub-diagrams, it is better to take the same node as the starting point in the whole construction. For the star graph with the center of 2, the effective Feynman diagram is given by

$$
V_{P}(2 ; 1 ш 3 \amalg 4 \amalg 5 ; 6)
$$

For the star graph with the center of 4 , when taking the node 2 as the starting point, we have following effective Feynman diagrams:

$$
\begin{array}{ll}
(0): V_{P}(2 ;\{4,1 ш 3 ш 5\} ; 6) ; & (1): V_{P}\left(2 ;\left\{P_{41}, 3 ш 5\right\} ; 6\right) \frac{1}{s_{41}} V_{C}\left(\left\{P_{41}, 1,4\right\}\right) \\
V_{P}\left(2 ;\left\{P_{43}, 1 ш 5\right\} ; 6\right) \frac{1}{s_{43}} V_{C}\left(\left\{P_{43}, 3,4\right\}\right), & V_{P}\left(2 ;\left\{P_{45}, 3 ш 1\right\} ; 6\right) \frac{1}{s_{45}} V_{C}\left(\left\{P_{45}, 5,4\right\}\right) ; \\
(2): V_{P}\left(2 ;\left\{P_{413}, 5\right\} ; 6\right) \frac{1}{s_{413}} V_{C}\left(\left\{P_{413}, 1,4,3\right\}\right), & V_{P}\left(2 ;\left\{P_{415}, 3\right\} ; 6\right) \frac{1}{s_{415}} V_{C}\left(\left\{P_{415}, 1,4,5\right\}\right), \\
V_{P}\left(2 ;\left\{P_{435}, 1\right\} ; 6\right) \frac{1}{s_{435}} V_{C}\left(\left\{P_{435}, 3,4,5\right\}\right) ; & \left.(3): V_{P}\left(2 ; P_{4135} ; 6\right) \frac{1}{s_{4135}} V_{P}(4 ; 1 ш 3 \amalg 5\} ; P_{4135}\right)
\end{array}
$$

Comparing above two sets of effective Feynman diagrams, we see immediately the common sub effective Feynman diagram is given by ${ }^{9}$

$$
V_{P}(2 ;\{4,1 \amalg 3 \amalg 5\} ; 6)
$$

which contains six cubic Feynman diagrams.

\section{$3 \quad$ Pick up Poles}

For a bi-adjoint scalar theory defined by two PT-factors, there is a way to extract a subset of all Feynman diagrams containing a particular pole structure. It is given in [19] by using following cross-ratio factor

$$
\mathcal{P}_{b d}^{a c}:=\frac{[a c][b d]}{[a d][b c]} \quad, \quad[a b]:=z_{a b}
$$

To be more explicitly, to pick up a specific pole $\frac{1}{s_{A}}$ from a given CHY-integrand composed by two ordered PT-factors, the subset $A$ must be contiguous subset in each PT-factor. Let us focus on just one of the PT-factor, there $a$ and $b$ to be the first and last elements of the subset $A$ respectively, while $c$ and $d$ are nearest elements of $a$ and $b$ respectively in the complement subset $\bar{A}$. Multiplying the cross-ratio factor (3.1) will reduce the index of possible poles containing nodes $a, c$ or nodes $b, d$ through numerators $[a c],[b d]$, while keeping the index of pole $s_{A}$ invariant. Although one can see that the denominator $[a d]$ and $[b c]$ from (3.1) will increase the index of poles containing nodes $a, d$ or nodes $b, c$, but since in the original PT-factor, there are no factors $[a d]$ and $[b c]$ in the denominator, their damage is under control. By these observations, one can show that multiplying (3.1) will

\footnotetext{
${ }^{9}$ There is a sign to be determined when CHY-integrands given by the multiplication of two different Cayley functions. It has been discussed in the paper [17].
} 

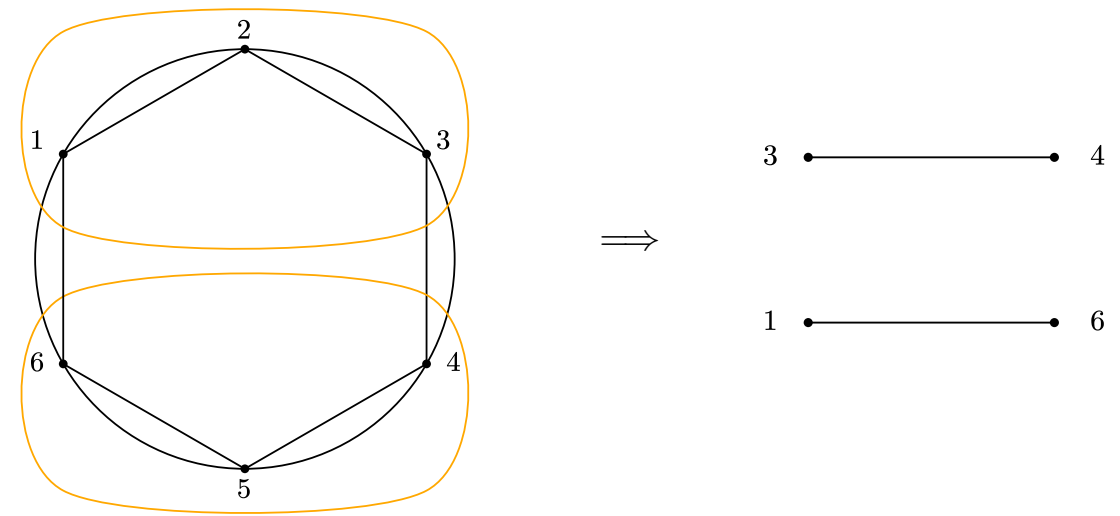

Figure 30. $\operatorname{Links}[\{1,2,3\},\{4,5,6\}]$ of integrand $\frac{1}{z_{12}^{2} z_{23}^{2} z_{34}^{2} z_{45}^{2} z_{56}^{2} z_{61}^{2}}$.

remove all Feynman diagrams having non-compatible poles, so we are left with diagrams all having the pole $s_{A}$. For example, with the CHY-integrand $\frac{1}{z_{12}^{2} z_{23}^{2} z_{34}^{2} z_{45}^{2} z_{56}^{2} z_{61}^{2}}$ we produce following fourteen Feynmann diagrams up to a sign

$$
\begin{aligned}
& \frac{1}{s_{12} s_{34} s_{56}}+\frac{1}{s_{12} s_{56} s_{123}}+\frac{1}{s_{23} s_{56} s_{123}}+\frac{1}{s_{12} s_{34} s_{126}}+\frac{1}{s_{16} s_{34} s_{126}}+ \\
& \frac{1}{s_{16} s_{23} s_{156}}+\frac{1}{s_{16} s_{34} s_{156}}+\frac{1}{s_{23} s_{56} s_{156}}+\frac{1}{s_{34} s_{56} s_{156}}+\frac{1}{s_{16} s_{23} s_{45}}+ \\
& \frac{1}{s_{12} s_{123} s_{45}}+\frac{1}{s_{23} s_{123} s_{45}}+\frac{1}{s_{12} s_{126} s_{45}}+\frac{1}{s_{16} s_{126} s_{45}} .
\end{aligned}
$$

To pick out all items containing $s_{123}$, first we split the whole set into the pole set $A=$ $\{1,2,3\}$ and its complement $\bar{A}=\{4,5,6\}$ as in the figure 30 . We see that there are lines connecting subsets $A$ and $\bar{A}$. For later convenience, we will define the $\operatorname{set} \operatorname{Links}[A, \bar{A}]$ as the collections of lines connecting the set $A$ and $\bar{A}$. Each line will be represented by two nodes: one is in $A$ and another one, in $\bar{A}$. Furthermore, we should distinguish the solid line(corresponding the factor $[a b]$ in the denominator) and dashed line(corresponding the factor $[a b]$ in the numerator) by $\{a, b\}$ and $\overline{\{a, b\}}$ respectively. Using this notation, we have

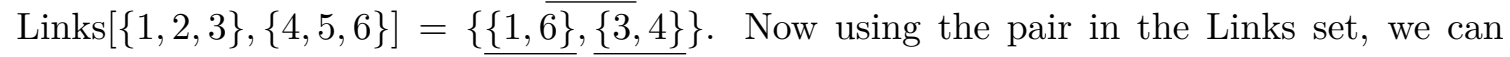
construct a single cross-ratio $\mathcal{P}_{34}^{16}=\frac{z_{16} z_{34}}{z_{14} z_{36}}$.

One can easily check that by multiplying such cross-ratio factor to the original CHYintegrand

$$
\frac{1}{z_{12}^{2} z_{23}^{2} z_{34}^{2} z_{45}^{2} z_{56}^{2} z_{61}^{2}} \mathcal{P}_{34}^{16}=\frac{1}{z_{12}^{2} z_{14} z_{16} z_{23}^{2} z_{34} z_{36} z_{45}^{2} z_{56}^{2}}
$$

we produce only four terms all containing the pole $s_{123}$

$$
\frac{1}{s_{12} s_{45} s_{123}}+\frac{1}{s_{23} s_{45} s_{123}}+\frac{1}{s_{12} s_{56} s_{123}}+\frac{1}{s_{23} s_{56} s_{123}}
$$

We are interesting in this problem is because in many situations, such as the soft limit and collinear limit, we want to know the singular behavior of a given amplitude. These singular behaviors are connected with particular poles, thus how to isolating contributions 
from these poles becomes important to many studies. In [19], by removing these singular contributions, the two-loop CHY-integrand of the planar bi-adjoing scalar theory has been constructed. In [18], the technique of picking poles has been used to study the symmetry properties of different PT-integrands. In [20], the same technique has been used to the contraction of the one-loop CHY-integrand for general bi-adjoing scalar theory.

In these mentioned applications, the pole picking is constraint to the CHY-integrands of two PT-factors. However, as presented in [1], a large class defined by the "labelled tree", which is the natural generalization of PT-factor, has been introduced. Thus it is curious that if the picking pole technique can be generalized to these more general situations. In this section, we would explore the possibility. We will give an algorithm to pick up a particular pole for the most general CHY-integrands, which do not contain any higher order pole. We must emphasize, unlike the case of PT-factors, where the picking pole algorithm can be rigorously proved, the general algorithm present in this section is based on many tested examples and we could not give the proof

Although we have talked only picking a given pole in this section, in fact, the procedure can continue to pick up more and more poles. When multiplying the cross-ratio factor, we get a new CHY-integrand and then from it, we continue to pick up another new pole. In other words, by iterating our algorithm, one can pick up a series of poles as long as they are compatible.

Another interesting point of picking pole technique is following. In the previous part, we have talked about the effective Feynman diagrams and the corresponding geometric picture, i.e., polytope. For the square Cayley integrand, all Feynman diagrams constitute a high-dimensional polytope. The process of picking out a particular pole corresponds precisely to the operation of projecting from a high-dimensional volume onto a specific face.

\subsection{The General algorithm}

Having discussed our purpose in this section, let us elaborate how to attack the problem. Unlike the bi-adjoint scalar theory, the general CHY-integrand of weight four contain both denominators and numerators and in general, amplitudes will depend on both, so the constructed cross-ratio factor of picking pole should depend on both too. Another important fact is that the index of a given pole is determined by links inside these nodes, thus the cross-ratio factor should not affect the index, which means lines used in the construction should come from these lines connecting the set $A$ and its complement $\bar{A}$. With this reasoning, one can see why we define the linking set $\operatorname{Links}[A, \bar{A}]$ in previous paragraphes.

Knowing the linking set $\operatorname{Links}[A, \bar{A}]$, in principle we can construct a cross-ratio factor like those in (3.1) for any two linking lines, which contain four nodes. ${ }^{10}$ However, in general case, there are two types of linking lines (i.e., the solid line representing the factor in denominator and the dashed line representing the factor in the numerator), it is understandable their roles should be different. Thus when we construct the cross-ratio factor, we should put their role into count. We will meet three situations. In the first situation

\footnotetext{
${ }^{10}$ If two nodes are the same point, the cross-ratio factor is automatically one.
} 
where both linking lines are solid lines, for example, $\{a, c\}$ and $\{b, d\}$, we can use (3.1) to define the cross-ratio factor by our experience from the bi-adjoint scalar theory. For later convenience, we will call them pure primary cross-ratio factor. In the second situation where both linking lines are dashed lines, for example, $\overline{\{a, c\}}$ and $\overline{\{b, d\}}$, the right definition of cross-ratio factor is not so straightforward. Naively one can use the definition (3.1), but now the role of solid lines and dashed lines are same. In fact, there is another definition, i.e., the inverse of (3.1), which we will write as

$$
\overline{\mathcal{P}}_{b d}^{a c} \equiv \frac{z_{a d} z_{b c}}{z_{a c} z_{b d}}=\left(\mathcal{P}_{b d}^{a c}\right)^{-1}
$$

In the new definition (3.5), the linking lines $\overline{\{a, c\}}$ and $\overline{\{b, d\}}$ are in denominator, so the difference between two kinds of lines is manifest. Although we feel the definition (3.5) is better, for all examples studied by us according to the algorithm presented below, the cross-ratio factor (3.5) will not appear.

The third situation is most tricky one, where one linking line is solid and another one, dashed, for example, $\overline{\{a, b\}}$ and $\{e, f\}$. Now, which definition, i.e., (3.1) and (3.5), we should use. In fact, in both definitions, the role of lines are same, which is not we want. We want an expression where the factor $[a b]$ is in the numerator while the factor $[e f]$ is in the denominator. However, using this pair, it is impossible to reach the goal. A way to solve the difficulty is to involve another linking line, for example, $\overline{\{c, d\}}$ and define following combination

$$
\mathcal{P}_{\underline{e f} ; \overline{a b}}^{\overline{a b}} \equiv \mathcal{P}_{c d}^{a b} \overline{\mathcal{P}}_{e f}^{c d}=\frac{z_{a b} z_{c d}}{z_{a d} z_{c b}} \frac{z_{e d} z_{c f}}{z_{e f} z_{c d}}=\frac{z_{a b} z_{e d} z_{c f}}{z_{e f} z_{a d} z_{c b}}
$$

We will call $\mathcal{P}_{e f ; \overline{c d}}^{\overline{a b}}$ the mixed primary cross-ratio factor. There is another possibility, i.e., involving $\{c, d\}$. Using this choice, we will get the inverse of (3.6) essentially. The reason we define (3.6) is that it does appear in the construction as shown in later examples. We must point out, although the line $\overline{\{c, d\}}$ acts as an intermediate variable, we do need to impose the condition $a \neq c, e \neq c$ and $b \neq d, f \neq d$.

Up to now, we have construct the (pure/mixed) primary cross-ratio factors, i.e., the (3.1), (3.6) and their inverses, by picking two or three lines from the linking set $\operatorname{Links}[A, \bar{A}]$. For general CHY-integrands, there will be many linking lines and we will have many primary cross-ratio factors. To pick up a given pole, we need to determine how to combine them. Based on many examples studied by us, now we present our algorithm in the following:

- (1) Given a CHY-integrand, draw the corresponding 4-regular graph, where the factor in the denominator is represented by solid line while the factor in the numerator is represented by dashed line.

- (2) To pick up the pole $s_{A}$, we divide all nodes of the graph into two subsets, i.e., the $A$ and its complement $\bar{A}$. Now there are lines connecting the subset $A$ and $\bar{A}$. Collecting them (by removing duplications) we form the linking set $\operatorname{Links}[A, \bar{A}]$. 
- (3) From the linking set $\operatorname{Links}[A, \bar{A}]$, we generate four collections of primary crossratio factors: (I) using two solid lines according to the formula (3.1); (II) using two dashed lines according to the formula (3.5); (III) Using two solid lines and one dashed line according to the formula (3.6). We want to emphasize that in the definition (3.6), the role of two solid lines are different, so we should construct two primary cross-ratio factors; (IV) Using two dashed lines and one solid line according to the inverse of the formula (3.6).

- (4) Now we use above four collections to construct the wanted cross-ratio factor, which picks up a particular pole. We start from the type (I) only. Assuming there are $N_{I}$ primary cross-ratio factors, we multiply them together to form the initial test pick-factor $\mathcal{P}_{0}$. Now we need to have some criterions to see if it is the right answer. The first criterion is that

- Criterion I: the new CHY-integrand, i.e, the multiplication of original CHYintegrand and the pick-factor, should not contain any new poles or higher order poles comparing with the original CHY-integrand.

It is worth to emphasize that when we say it does not create new poles or higher poles, we are just calculate the pole index of a subset $A_{i}$ as defined in (1.5) If the $\mathcal{P}_{0}$ satisfies the Criterion I, it is the right answer (see examples given in 3.2.1).

- (5) If the $\mathcal{P}_{0}$ in the above item does not satisfy the Criterion I, we should consider factors obtained by removing one primary cross-ratio factor from $\mathcal{P}_{0}$. In other words, among $N_{I}$ primary cross-ratio factors in the collection (I), we choose arbitrary $\left(N_{I}-1\right)$ of them and multiply them together. There will be $N_{I}$ different choices. For each choice, we check if it satisfies the Criterion I. If it violates the Criterion I, we just discard it. Otherwise, we keep it.

Assuming that there are $1 \leq m_{1} \leq N_{I}$ left combinations in the list, now we need to impose the second criterion:

- Criterion II: the pick-factor should remove all incompatible poles of the original integrand.

More explicitly, for each pick-factor in the list, we calculate the remaining poles (by just calculating the pole index) after multiplying it to the original CHY-integrand. From it, we can find the kept poles for each pick-factor. We claim that the right pick-factors are those that all incompatible poles have been removed. There may be several combinations satisfying the Criterion II. Each one will be the right choice of pick-factors (see examples in 3.2.2).

- (6) If there is no combination satisfying the Criterion I by removing just one primary cross-ratio factor from $\mathcal{P}_{0}$, i.e., $m_{1}=0$ in previous item. We continue to consider the situation by removing two primary cross-ratio factors from $\mathcal{P}_{0}$. Again we consider various allowed combinations and check them with the Criterion I. If there are combinations left after this checking, we impose the Criterion II on these left combination. 
If there is no combination after this checking, we continue to remove three primary cross-ratio factors from $\mathcal{P}_{0}$ and checking with Criterion I and II.

Now one sees the iterating procedure. At the end with primary cross-ratio factors in the collection (I), we will face two situations: either finding the right pick-factor according to our algorithm (see examples in 3.2.3), or either there is no combination satisfying both Criterions.

- (7) When we can not find the right pick-factor just from the collection (I), we need to enlarge our pool by including the collection (III). For the enlarged collection, we repeat the procedure from the item (4) to item (6). However, since in general the number of element in the collection (III) is large and the final answer contains very small number of factors from the collection (III), based on experience with many examples we find it is more effective doing following way:

- (7a) First we collect all cross-ratio factors obtained from the multiplication of the primary cross-ratio factors in the collection (I), which satisfy the Criterion I. ${ }^{11}$ We denote them as $\mathcal{T}_{i ; k}$ where $i$ is the number of primary cross-ratio factors in the multiplication and $k$ distinguishes different combinations with same number $i$. The allowed choices of $k$ will be denoted as $N_{i}$.

- (7b) Secondly, we denote elements in the collection (III) by $a_{i}, i=1, \ldots, M_{I I I}$.

- (7c) Now we consider following combination

$$
\mathcal{T}_{i ; k} \prod_{1 \leq i_{1}<\ldots<i_{m} \leq M_{I I I}} a_{i_{1}} \ldots a_{i_{m}}
$$

We check cross-ratio factor by the Criterion I and II in (3.7) according to following ordering. When searching through the combination (3.7), there are four variables. The first one is the changing of the index $i$. The second one is the changing of the index $k$. The third one is the number $m$ and the fourth one is the different choices of a given number $m$. In other words, there are four nested loops in the searching and one can arrange the searching orderings of four variables according to his preference.

Our choice is to start from the smallest number $m=1$ and the largest number $i$, then we change $k=1, \ldots, N_{i}$ and different $i_{1}$. If there are combinations satisfy both criterions, we find the right pick-factor. If there is no combinations satisfy both, we go to the next largest number $i$ and search for all combinations of $k$ and $i_{1}$. If after going through all $i$ with fixed $m=1$ we do not find the right pick-factor, we move to the case $m=2$ and start from largest $i$ to smallest $i$ again. In other words, for the four nested loops. The outmost loop is $m=1$

\footnotetext{
${ }^{11}$ It is possible that the combination in collection (I), which does not satisfy the Criterion I, will give the right pick-factor when multiplying some factors from the collection (III). The more effective algorithm presented here has overlooked this possibility. Thus if it does not work, we should go back to the more complete algorithm, i.e., we repeat the procedure from the item (4) to item (6) for the collections (I) plus (III).
} 
to $m=M_{I I I}$. The next loop is from largest $i$ to smallest $i$. The third loop is the different choice of $k$ and the innermost loop is different combinations of $a_{i_{1}} \ldots a_{i_{m}}$ with given $m$.

- (8) Although by our explicit examples, there is no need to include the collections (II) and (IV), but when the step (4) to step (7) fail, this possibility should be considered.

Having presented the algorithm above, let us give the motivations of the construction without the rigorous proof. First, since all primary cross-ratio factors are constructed from links between $A$ and $\bar{A}$, multiplying them to the original CHY-integrand will not change the linking number of the subset $A$ and any other subsets $B \subset A$. In other words, any compatible pole of $s_{A}$ in the original theory will be kept. A good point is that at the same time, these cross-ratio factors will remove some incompatible poles. Naively, since each factor will remove part of incompatible poles, multiplying them together will remove all incompatible poles. However, while getting rid old incompatible poles, it could produce new incompatible poles. Thus we should impose the Criterion I. Furthermore, if we do not multiply all factor together, it will have the chance that some incompatible poles are left, thus we need to impose the Criterion II to remove this possibility (see example in the figure 34).

Before ending the subsection, let us give some technical remarks. As in general discussion in the previous paragraph, to pick a given pole, we need to remove all incompatible poles by multiplying some cross-ratio factors while not add new poles (i.e., the Criterion I and II). To reach the goal, some understanding of primary cross-ratio factors is very useful. For the primary factor in the collection (I) and (II), the general form is

$$
\frac{[a b][c d]}{[a d][c b]}
$$

From this form, we see that this factor will remove poles containing nodes in any one of following two groups

$$
\{a, b\} ; \quad\{c, d\}
$$

and has the potential to add new poles containing nodes in any one of following two groups

$$
\{a, d\} ; \quad\{c, b\}
$$

For the primary factor in the collection (III) and (IV), the general form is

$$
\frac{[a b][c f][e d]}{[a d][c b][e f]}
$$

From this form, we see that this factor will remove poles containing nodes in any one of following six groups

$$
\{a, b\} ; \quad\{c, f\} ; \quad\{e, d\} ; \quad\{a, b, c, f\} ; \quad\{a, b, e, d\} ; \quad\{c, f, e, d\}
$$

while has the potential to add new poles containing in any one of following six groups

$$
\{a, d\} ; \quad\{c, b\} ; \quad\{e, f\} ; \quad\{a, d, c, b\} ; \quad\{a, d, e, f\} ; \quad\{c, b, e, f\}
$$

Using information (3.9), (3.10), (3.12) and (3.13), we can speed up the check with the Criterion I and II through our algorithm laid above. 


\subsection{Examples}

In this part, we will present various examples to demonstrate our algorithm.

\subsubsection{The simplest case}

The simplest case is that we have used only the collection (I) and multiplications of all its factors satisfies the Criterion I. As we have claimed, the obtained cross-ratio factor is the right answer. We will present two examples. The first example is the Hamilton star graph

$$
\mathcal{I}_{6}^{\text {star }}=\frac{z_{16}^{4}}{z_{12}^{2} z_{13}^{2} z_{14}^{2} z_{15}^{2} z_{26}^{2} z_{36}^{2} z_{46}^{2} z_{56}^{2}}
$$

It gives a basic effective vertex as discussed in the first part of the paper. This effective vertex can be expanded to $4 !=24$ cubic Feynman diagrsms

$$
\begin{aligned}
& \frac{1}{s_{12} s_{36} s_{124}}+\frac{1}{s_{14} s_{36} s_{124}}+\frac{1}{s_{12} s_{56} s_{124}}+\frac{1}{s_{14} s_{56} s_{124}}+\frac{1}{s_{12} s_{36} s_{125}}+ \\
& \frac{1}{s_{15} s_{36} s_{125}}+\frac{1}{s_{12} s_{46} s_{125}}+\frac{1}{s_{15} s_{46} s_{125}}+\frac{1}{s_{13} s_{26} s_{134}}+\frac{1}{s_{14} s_{26} s_{134}}+ \\
& \frac{1}{s_{13} s_{56} s_{134}}+\frac{1}{s_{14} s_{56} s_{134}}+\frac{1}{s_{13} s_{26} s_{135}}+\frac{1}{s_{15} s_{26} s_{135}}+\frac{1}{s_{13} s_{46} s_{135}}+ \\
& \frac{1}{s_{15} s_{46} s_{135}}+\frac{1}{s_{14} s_{26} s_{145}}+\frac{1}{s_{15} s_{26} s_{145}}+\frac{1}{s_{14} s_{36} s_{145}}+\frac{1}{s_{15} s_{36} s_{145}}+ \\
& \frac{1}{s_{12} s_{46} s_{123}}+\frac{1}{s_{13} s_{46} s_{123}}+\frac{1}{s_{12} s_{56} s_{123}}+\frac{1}{s_{13} s_{56} s_{123}}
\end{aligned}
$$

Let us try to pick the pole $s_{123}$. The corresponding node-set would be $A=\{1,2,3\}, \bar{A}=$ $\{4,5,6\}$ as shown in the figure 31 . There are four solid lines connecting $A, \bar{A}$, i.e., $\{1,4\}$, $\{1,5\},\{2,6\}$ and $\{3,6\}$. From them, we can construct six primary cross-ratio factors, but two of them are just trivial one:

$$
\begin{aligned}
& \mathcal{P}_{26}^{14}=\frac{z_{14} z_{26}}{z_{16} z_{24}} \quad \mathcal{P}_{36}^{14}=\frac{z_{14} z_{36}}{z_{16} z_{34}} \quad \mathcal{P}_{15}^{14}=1 \\
& \mathcal{P}_{26}^{15}=\frac{z_{15} z_{26}}{z_{16} z_{25}} \quad \mathcal{P}_{36}^{15}=\frac{z_{15} z_{36}}{z_{16} z_{35}} \quad \mathcal{P}_{36}^{26}=1
\end{aligned}
$$

When multiplying them together, we get $\mathcal{P}_{0}=\frac{z_{14}^{2} z_{15}^{2} z_{26}^{2} z_{36}^{2}}{z_{16}^{4} z_{24} z_{25} z_{34} z_{35}}$. It is easy to check that $\mathcal{P}_{0}$ satisfies the Criterion I, so it is the right factor. One can easily check that the new CHY-integrand

$$
\mathcal{I}_{6}^{\text {star }} \frac{z_{14}^{2} z_{15}^{2} z_{26}^{2} z_{36}^{2}}{z_{16}^{4} z_{24} z_{25} z_{34} z_{35}}=\frac{1}{z_{12}^{2} z_{13}^{2} z_{24} z_{25} z_{34} z_{35} z_{46}^{2} z_{56}^{2}}
$$

will produce the amplitudes

$$
\frac{1}{s_{12} s_{46} s_{123}}+\frac{1}{s_{13} s_{46} s_{123}}+\frac{1}{s_{12} s_{56} s_{123}}+\frac{1}{s_{13} s_{56} s_{123}}
$$

which does pick up all terms containing the pole $s_{123}$. We verify that for all the star graphs with $n \leq 8$ the algorithm is right. 



Figure 31. The partition of the integrand $\frac{z_{16}^{4}}{z_{12}^{2} z_{13}^{2} z_{14}^{2} z_{15}^{2} z_{26}^{2} z_{36}^{2} z_{46}^{2} z_{56}^{2}}$ and the corresponding links be-
tween two parts

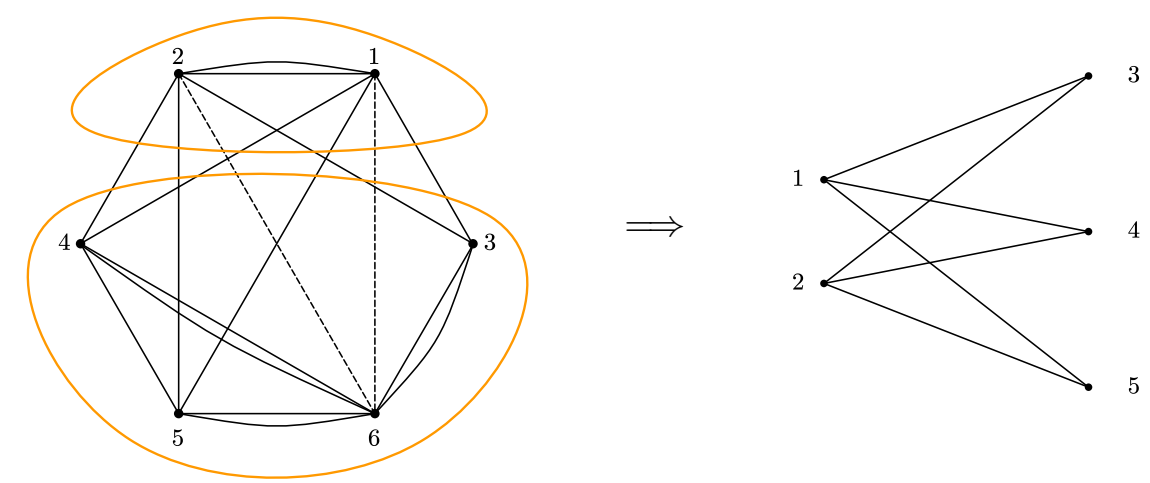

Figure 32. $\operatorname{Links}[\{1,2\},\{3,4,5,6\}]$ of integrand $\frac{z_{16} z_{26}}{z_{12}^{2} z_{13} z_{14} z_{15} z_{23} z_{24} z_{25} z_{36}^{2} z_{46}^{2} z_{56}^{2}}$

Another example is following. The CHY-integrand ${ }^{12}$

$$
\mathcal{I}_{6}^{\prime}=\frac{z_{16} z_{26}}{z_{12}^{2} z_{13} z_{14} z_{15} z_{23} z_{24} z_{25} z_{36}^{2} z_{46}^{2} z_{56}^{2}}
$$

gives the amplitude

$$
-\frac{1}{s_{12} s_{56} s_{123}}-\frac{1}{s_{12} s_{36} s_{124}}-\frac{1}{s_{12} s_{56} s_{124}}-\frac{1}{s_{12} s_{36} s_{125}}-\frac{1}{s_{12} s_{46} s_{125}}-\frac{1}{s_{12} s_{46} s_{123}}
$$

where pole $s_{12}$ appears in every term. Now we want to pick pole $s_{12}$. From the figure 32, we see that there are six solid lines connecting $A=\{1,2\}, \bar{A}=\{3,4,5,6\}$. Among 15 primary cross-ratio factors of collection (I), only following six are nontrivial.

$$
\begin{array}{lll}
\mathcal{P}_{24}^{13}=\frac{z_{13} z_{24}}{z_{14} z_{23}}, & \mathcal{P}_{25}^{13}=\frac{z_{13} z_{25}}{z_{15} z_{23}}, & \mathcal{P}_{23}^{14}=\frac{z_{14} z_{23}}{z_{13} z_{24}} \\
\mathcal{P}_{25}^{14}=\frac{z_{14} z_{25}}{z_{15} z_{24}}, & \mathcal{P}_{23}^{15}=\frac{z_{15} z_{23}}{z_{13} z_{25}}, & \mathcal{P}_{24}^{15}=\frac{z_{15} z_{24}}{z_{14} z_{25}}
\end{array}
$$

\footnotetext{
${ }^{12}$ In fact, this CHY-integrand is obtained from the star graph $\mathcal{I}_{6}=\frac{z_{26}^{2}}{z_{12}^{2} z_{13}^{2} z_{24}^{2} z_{25}^{2} z_{36}^{2} z_{46}^{2} z_{56}^{2}}$ by multiplying the cross-ratio factor $\frac{z_{13} z_{16} z_{24} z_{25}}{z_{14} z_{15} z_{23} z_{26}}$ of picking up pole $s_{12}$ according to our algorithm.
} 
Now according to our algorithm, we multiply them together. Amazingly, we get

$$
\mathcal{P}_{24}^{13} \mathcal{P}_{25}^{13} \mathcal{P}_{23}^{14} \mathcal{P}_{25}^{14} \mathcal{P}_{23}^{15} \mathcal{P}_{24}^{15}=1
$$

Although 1 is a trivial factor at the right hand side, the left hand side is nontrivial combination. The result tells us that to pick up terms containing the pole $s_{12}$, the cross-ratio factor we need to multiply is just one as we have expected.

\subsubsection{The next simplest examples}

In this part, we present some examples where pick-pole factors are multiplication of primary cross-ratio factor in the collection (I), but not with the maximum number. In this case, we need to impose the Criterion I and II.

The first example is the CHY-integrand

$$
\mathcal{I}_{6}^{[1]}=\frac{1}{z_{12}^{2} z_{23}^{2} z_{34}^{2} z_{56}^{2} z_{45} z_{61} z_{46} z_{15}}
$$

which produce following five cubic Feynman diagrams (all terms with common pole $s_{56}$ )

$$
\frac{1}{s_{12} s_{34} s_{56}}+\frac{1}{s_{12} s_{123} s_{56}}+\frac{1}{s_{23} s_{123} s_{56}}+\frac{1}{s_{23} s_{156} s_{56}}+\frac{1}{s_{34} s_{156} s_{56}}
$$

To pick pole $s_{12}$, first we get three solid lines connecting $A=\{1,2\}, \bar{A}=\{3,4,5,6\}$ as $\{2,3\},\{1,6\}$ and $\{1,5\}$. From it, we get the primary cross-ratio factors:

$$
\mathcal{P}_{16}^{23}=\frac{z_{16} z_{23}}{z_{13} z_{26}}, \quad \mathcal{P}_{15}^{23}=\frac{z_{15} z_{23}}{z_{13} z_{25}}, \quad \mathcal{P}_{15}^{16}=1
$$

Multiplying them together we get $\mathcal{P}_{16}^{23} \mathcal{P}_{15}^{23}=\frac{z_{15} z_{16} z_{23}^{2}}{z_{13}^{2} z_{25} z_{26}}$. It is easy to see that it violates the Criterion I because of the appearance new pole $s_{13}$. Now we remove one primary cross-ratio factor and get two choices

$$
\mathcal{I}[1]_{s_{12}}=\mathcal{I}_{6}^{[1]} \mathcal{P}_{16}^{23}, \quad \mathcal{I}[2]_{s_{12}}=\mathcal{I}_{6}^{[1]} \mathcal{P}_{15}^{23}
$$

It can be checked that both satisfy the Criterion II and are right answer. Indeed they produce the same amplitude

$$
\frac{1}{s_{12} s_{34} s_{56}}+\frac{1}{s_{12} s_{123} s_{56}}
$$

The next example is more tricky. The CHY-integrand

$$
\mathcal{I}_{6}^{[2]}=\frac{z_{46}^{2}}{z_{13} z_{14} z_{16}^{2} z_{24} z_{25} z_{26}^{2} z_{34}^{2} z_{36} z_{45}^{2} z_{56}}
$$

produces following six cubic Feynman diagrams:

$$
\frac{1}{s_{26} s_{34} s_{126}}+\frac{1}{s_{16} s_{45} s_{126}}+\frac{1}{s_{26} s_{45} s_{126}}+\frac{1}{s_{26} s_{34} s_{134}}+\frac{1}{s_{16} s_{45} s_{136}}+\frac{1}{s_{16} s_{34} s_{126}}
$$

containing $\left\{s_{16}, s_{26}, s_{34}, s_{45}, s_{126}, s_{134}, s_{136}\right\}$ seven poles. Now we want to pick pole $s_{136}$. 




Figure 33. The partition of the CHY-integrand lines connecting the two groups.

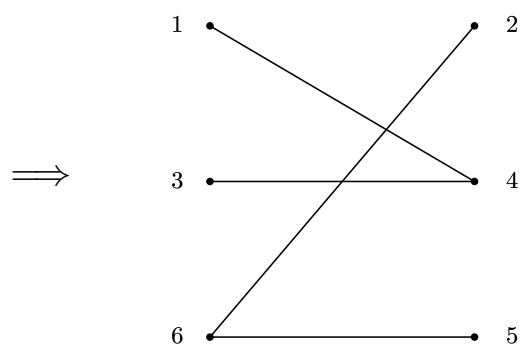
$\frac{z_{46}^{2}}{z_{13} z_{14} z_{16}^{2} z_{24} z_{25} z_{26}^{2} z_{34}^{2} z_{36} z_{45}^{2} z_{56}}$ and the corresponding $z_{13} z_{14} z_{16}^{2} z_{24} z_{25} z_{26}^{2} z_{34}^{2} z_{36} z_{45}^{2} z_{56}$

\begin{tabular}{|ll|}
\hline $\mathcal{I}$ & Kept Poles \\
\hline $\mathcal{I}_{6}^{[2]} \mathcal{P}_{26}^{14} \mathcal{P}_{56}^{14} \mathcal{P}_{34}^{26}=\frac{z_{14}}{z_{12} z_{13} z_{15} z_{16}^{2} z_{23} z_{24} z_{25} z_{34} z_{36} z_{45}^{2} z_{46}}$ & $\left\{s_{16}, s_{45}, s_{136}\right\}$ \\
$\mathcal{I}_{6}^{[2]} \mathcal{P}_{26}^{14} \mathcal{P}_{56}^{14} \mathcal{P}_{56}^{34}=\frac{z_{14} z_{56}}{z_{12} z_{13} z_{15} z_{16}^{2} z_{24} z_{25} z_{26} z_{34} z_{35} z_{36} z_{45}^{2} z_{46}}$ & $\left\{s_{16}, s_{45}, s_{126}, s_{136}\right\}$ \\
$\mathcal{I}_{6}^{[2]} \mathcal{P}_{26}^{14} \mathcal{P}_{34}^{26} \mathcal{P}_{56}^{34}=\frac{1}{z_{12} z_{13} z_{16}^{2} z_{23} z_{24} z_{25} z_{35} z_{36} z_{45}^{2} z_{46}}$ & $\left\{s_{16}, s_{45}, s_{136}\right\}$ \\
$\mathcal{I}_{6}^{[2]} \mathcal{P}_{56}^{14} \mathcal{P}_{34}^{26} \mathcal{P}_{56}^{34}=\frac{z_{56}}{z_{13} z_{15} z_{16}^{2} z_{23} z_{24} z_{25} z_{26} z_{35} z_{36} z_{45}^{2} z_{46}}$ & $\left\{s_{16}, s_{45}, s_{136}\right\}$ \\
\hline
\end{tabular}

Figure 34. All the combinations without add new poles of (3.31).

From the figure 33 we see there are for solid lines $\{1,4\},\{1,6\},\{3,4\}$ and $\{5,6\}$ connecting to $A=\{1,3,6\}, \bar{A}=\{2,4,5\}$, which produce four primary cross-ratio factors

$$
\mathcal{P}_{26}^{14}=\frac{z_{14} z_{26}}{z_{12} z_{46}}, \quad \mathcal{P}_{56}^{14}=\frac{z_{14} z_{56}}{z_{15} z_{46}}, \quad \mathcal{P}_{34}^{26}=\frac{z_{26} z_{34}}{z_{23} z_{46}}, \quad \mathcal{P}_{56}^{34}=\frac{z_{34} z_{56}}{z_{35} z_{46}}
$$

It is easy to see that multiplying four together, we will get $\frac{z_{14}^{2} z_{26}^{2} z_{34}^{2} z_{56}^{2}}{z_{12} z_{15} z_{23} z_{35} z_{46}^{4}}$, which will produce new pole $s_{46}$ when multiplying the original CHY-integrand. Thus by our algorithm, we need to consider combinations with only three primary cross-ratio factors. There are four choices. When multiplying with $\mathcal{I}_{6}^{[3]}$, the kept poles are listed in following figure 34 . We see that all four choices satisfy the Criterion I, but when checking the kept poles, one of them contains incompatible pole $s_{126}$. Thus by our Criterion II, the right answer is other three combinations. One can easily check that all three combination do produce the same amplitude $\frac{1}{s_{16} s_{45} s_{136}}$ as we expected.

\subsubsection{More complicated examples}

In this subsubsection, we present examples with removing more than just one primary cross-ratio factors in the collection (I). The first example is the familiar bi-adjoint scalar theory with the CHY-integrand coming from the product of two PT-factors

$$
\mathcal{I}_{6}^{[3]}=\frac{1}{z_{13}^{2} z_{14} z_{16} z_{24} z_{25}^{2} z_{26} z_{34} z_{36} z_{45} z_{56}}
$$


It provide following two Feynman diagrams, where the only difference between the two is poles $s_{134}$ and $s_{136}$ :

$$
\frac{1}{s_{13} s_{25} s_{134}}+\frac{1}{s_{13} s_{25} s_{136}}
$$

For this example, in previous literature [19], we have given the algorithm to pick up a particular pole. Now we will see how the old algorithm is included in our new algorithm.

To pick the pole $s_{134}$, first we find four solid lines connecting $A=\{1,3,4\}, \bar{A}=\{2,5,6\}$, i.e., $\{1,6\},\{4,2\},\{3,6\}$ and $\{4,5\}$. From it, we get four nontrivial primary cross-ratio factors:

$$
\mathcal{P}_{24}^{16}=\frac{z_{16} z_{24}}{z_{12} z_{46}}, \quad \mathcal{P}_{36}^{24}=\frac{z_{24} z_{36}}{z_{23} z_{46}}, \quad \mathcal{P}_{45}^{16}=\frac{z_{16} z_{45}}{z_{15} z_{46}}, \quad \mathcal{P}_{45}^{36}=\frac{z_{36} z_{45}}{z_{35} z_{46}}
$$

Multiplying them together we get $\mathcal{P}_{24}^{16} \mathcal{P}_{45}^{16} \mathcal{P}_{36}^{24} \mathcal{P}_{45}^{36}=\frac{z_{16} z_{24} z_{36} z_{45}}{z_{12} z_{13}^{2} z_{14} z_{15} z_{23} z_{25}^{2} z_{26} z_{34} z_{35} z_{46}^{4} z_{56}}$. It has added the new poles $s_{123}, s_{125}, s_{135}$ and $s_{146}$, which violate the Criterion I. Next, we need to remove one of four primary cross-ratio factors in turn to check with our criterion. We have shown such process in the figure 35. From it we could see that it is not enough to remove just one primary cross-ratio factor. To get the pick-factor without adding new poles, we need to remove three factors. In the end to satisfy the Criterion I we can only keep one primary cross-ratio factor in (3.33). One can check that each of them produces the same right amplitude

$$
\frac{1}{s_{13} s_{25} s_{134}}
$$

Next, we consider another CHY-integrand which do not belong to the category of Cayley tree

$$
\mathcal{I}_{8}^{[4]}=\frac{z_{18}^{2} z_{28} z_{36}}{z_{12} z_{15}^{2} z_{16}^{2} z_{17} z_{23}^{2} z_{24} z_{26} z_{34} z_{37} z_{38} z_{45} z_{48} z_{58} z_{68}^{2} z_{78}^{2}}
$$

It gives seven cubic Feynman diagrams

$$
\begin{aligned}
& \frac{1}{s_{16} s_{23} s_{78} s_{156} s_{234}}+\frac{1}{s_{15} s_{23} s_{68} s_{234} s_{678}}+\frac{1}{s_{15} s_{23} s_{78} s_{234} s_{678}}-\frac{1}{s_{16} s_{78} s_{126} s_{378} s_{1256}} \\
& -\frac{1}{s_{15} s_{78} s_{156} s_{378} s_{1256}}-\frac{1}{s_{16} s_{78} s_{156} s_{378} s_{1256}}+\frac{1}{s_{15} s_{23} s_{78} s_{156} s_{234}}
\end{aligned}
$$

with an important property, i.e., different terms have relative sign.

To pick the pole $s_{15}$, first we write down five solid lines connecting $A=\{1,5\}, \bar{A}=$ $\{2,3,4,5,6\}$, i.e., $\{1,2\},\{1,6\},\{1,7\},\{5,4\}$ and $\{5,8\}$. From it, we get six nontrivial primary cross-ratio factors:

$$
\begin{array}{lll}
\mathcal{P}_{45}^{12}=\frac{z_{12} z_{45}}{z_{14} z_{25}}, & \mathcal{P}_{45}^{16}=\frac{z_{16} z_{45}}{z_{14} z_{56}}, & \mathcal{P}_{45}^{17}=\frac{z_{17} z_{45}}{z_{14} z_{57}} \\
\mathcal{P}_{58}^{16}=\frac{z_{16} z_{58}}{z_{18} z_{56}}, & \mathcal{P}_{58}^{17}=\frac{z_{17} z_{58}}{z_{18} z_{57}}, & \mathcal{P}_{58}^{12}=\frac{z_{12} z_{58}}{z_{18} z_{25}}
\end{array}
$$

Multiplying them together we get $\mathcal{P}_{45}^{12} \mathcal{P}_{58}^{12} \mathcal{P}_{45}^{16} \mathcal{P}_{58}^{16} \mathcal{P}_{45}^{17} \mathcal{P}_{58}^{17}=\frac{z_{12}^{2} z_{16}^{2} z_{17}^{2} z_{45}^{3} z_{58}^{3}}{z_{14}^{3} z_{18}^{3} z_{25}^{2} z_{56}^{2} z_{57}^{2}}$. It would added the new poles $s_{25}, s_{56}$ and $s_{57}$, thus violates the Criterion I. Next, we need to remove one or more of six primary cross-ratio factors in turn to check with our criterion. To get the 


\begin{tabular}{|c|c|}
\hline $\mathcal{I}$ & Added Poles \\
\hline $\mathcal{I}_{6}^{[3]} \mathcal{P}_{24}^{16} \mathcal{P}_{45}^{16} \mathcal{P}_{36}^{24} \mathcal{P}_{45}^{36}=\frac{z_{16} z_{24} z_{36} z_{45}}{z_{12} z_{13}^{2} z_{14} z_{15} z_{23} z_{25}^{2} z_{26} z_{34} z_{35} z_{46}^{4} z_{56}}$ & $\left\{s_{123}, s_{125}, s_{135}, s_{146}\right\}$ \\
\hline $\mathcal{I}_{6}^{[3]} \mathcal{P}_{45}^{16} \mathcal{P}_{36}^{24} \mathcal{P}_{45}^{36}=\frac{z_{36} z_{45}}{z_{13}^{2} z_{14} z_{15} z_{23} z_{25}^{2} z_{26} z_{34} z_{35} z_{46}^{3} z_{56}}$ & $\left\{s_{135}, s_{146}\right\}$ \\
\hline $\mathcal{I}_{6}^{[3]} \mathcal{P}_{24}^{16} \mathcal{P}_{45}^{16} \mathcal{P}_{45}^{36}=\frac{z_{16} z_{45}}{z_{12} z_{13}^{2} z_{14} z_{15} z_{25}^{2} z_{26} z_{34} z_{35} z_{46}^{3} z_{56}}$ & $\left\{s_{125}, s_{135}\right\}$ \\
\hline $\mathcal{I}_{6}^{[3]} \mathcal{P}_{24}^{16} \mathcal{P}_{36}^{24} \mathcal{P}_{45}^{36}=\frac{z_{24} z_{36}}{z_{12} z_{13}^{2} z_{14} z_{23} z_{25}^{2} z_{26} z_{34} z_{35} z_{46}^{3} z_{56}}$ & $\left\{s_{123}, s_{146}\right\}$ \\
\hline $\mathcal{I}_{6}^{[3]} \mathcal{P}_{24}^{16} \mathcal{P}_{45}^{16} \mathcal{P}_{36}^{24}=\frac{z_{16} z_{24}}{z_{12} z_{13}^{2} z_{14} z_{15} z_{23} z_{25}^{2} z_{26} z_{34} z_{46}^{3} z_{56}}$ & $\left\{s_{123}, s_{125}\right\}$ \\
\hline $\mathcal{I}_{6}^{[3]} \mathcal{P}_{45}^{16} \mathcal{P}_{45}^{36}=\frac{z_{45}}{z_{13}^{2} z_{14} z_{15} z_{24} z_{25}^{2} z_{26} z_{34} z_{35} z_{46}^{2} z_{56}}$ & $\left\{s_{46}, s_{135}\right\}$ \\
\hline $\mathcal{I}_{6}^{[3]} \mathcal{P}_{36}^{24} \mathcal{P}_{45}^{36}=\frac{z_{36}}{z_{13}^{2} z_{14} z_{16} z_{23} z_{25}^{2} z_{26} z_{34} z_{35} z_{46}^{2} z_{56}}$ & $\left\{s_{46}, s_{146}\right\}$ \\
\hline $\mathcal{I}_{6}^{[3]} \mathcal{P}_{45}^{16} \mathcal{P}_{36}^{24}=\frac{1}{z_{13}^{2} z_{14} z_{15} z_{23} z_{25}^{2} z_{26} z_{34} z_{46}^{2} z_{56}}$ & $\left\{s_{46}\right\}$ \\
\hline $\mathcal{I}_{6}^{[3]} \mathcal{P}_{24}^{16} \mathcal{P}_{45}^{36}=\frac{1}{z_{12} z_{13}^{2} z_{14} z_{25}^{2} z_{26} z_{34} z_{35} z_{46}^{2} z_{56}}$ & $\left\{s_{46}\right\}$ \\
\hline $\mathcal{I}_{6}^{[3]} \mathcal{P}_{24}^{16} \mathcal{P}_{45}^{16}=\frac{z_{16}}{z_{12} z_{13}^{2} z_{14} z_{15} z_{25}^{2} z_{26} z_{34} z_{36} z_{46}^{2} z_{56}}$ & $\left\{s_{46}, s_{125}\right\}$ \\
\hline $\mathcal{I}_{6}^{[3]} \mathcal{P}_{24}^{16} \mathcal{P}_{36}^{24}=\frac{z_{24}}{z_{12} z_{13}^{2} z_{14} z_{23} z_{25}^{2} z_{26} z_{34} z_{45} z_{46}^{2} z_{56}}$ & $\left\{s_{46}, s_{123}\right\}$ \\
\hline $\mathcal{I}_{6}^{[3]} \mathcal{P}_{45}^{36}=\frac{1}{z_{13}^{2} z_{14} z_{16} z_{24} z_{25}^{2} z_{26} z_{34} z_{35} z_{46} z_{56}}$ & \{\} \\
\hline $\mathcal{I}_{6}^{[3]} \mathcal{P}_{45}^{16}=\frac{1}{z_{13}^{2} z_{14} z_{15} z_{24} z_{25}^{2} z_{26} z_{34} z_{36} z_{46} z_{56}}$ & \{\} \\
\hline $\mathcal{I}_{6}^{[3]} \mathcal{P}_{36}^{24}=\frac{1}{z_{13}^{2} z_{14} z_{16} z_{23} z_{25}^{2} z_{26} z_{34} z_{45} z_{46} z_{56}}$ & \{\} \\
\hline $\mathcal{I}_{6}^{[3]} \mathcal{P}_{24}^{16}=\frac{1}{z_{12} z_{13}^{2} z_{14} z_{25}^{2} z_{26} z_{34} z_{3}}$ & \{\} \\
\hline
\end{tabular}

Figure 35. All the combinations without add new poles of (3.31).

pick-factor without adding new poles, we need to remove at least three factors as shown in the figure 36. For the cross-ratio factors, which satisfy the Criterion I in the figure 36, we check if they meet Criterion II as shown in the figure 37. Thus they are the wanted pick-factor. Direct calculation shows that they all produces the same right amplitude:

$$
\frac{1}{s_{15} s_{23} s_{68} s_{234} s_{678}}+\frac{1}{s_{15} s_{23} s_{78} s_{234} s_{678}}-\frac{1}{s_{15} s_{78} s_{156} s_{378} s_{1256}}+\frac{1}{s_{15} s_{23} s_{78} s_{156} s_{234}}
$$

One thing we want to emphasize is that these pick factors keep the signs among different terms.

\subsection{The pick-factors with mixed type of cross-ratio factors}

In this subsubsection, we will present several examples where pick-factors are constructed using both collection (I) and (III).

The first example is the CHY-integrand of 8-point

$$
\mathcal{I}_{8}=\frac{z_{38}^{2} z_{78}^{2}}{z_{17}^{2} z_{18}^{2} z_{23}^{2} z_{28}^{2} z_{35}^{2} z_{37}^{2} z_{46}^{2} z_{48}^{2} z_{58}^{2} z_{67}^{2}}
$$




\begin{tabular}{|c|c|}
\hline $\mathcal{I}$ & \# of Added Poles \\
\hline $\mathcal{I}^{[4]} \mathcal{P}_{58}^{12} \mathcal{P}_{58}^{16} \mathcal{P}_{58}^{17}=\frac{z_{28} z_{36} z_{58}^{2}}{z_{15}^{2} z_{16} z_{18} z_{23}^{2} z_{24} z_{25} z_{26} z_{34} z_{37} z_{38} z_{45} z_{48} z_{56} z_{57} z_{68}^{2} z_{78}^{2}}$ & 2 \\
\hline $\mathcal{I}^{[4]} \mathcal{P}_{58}^{16} \mathcal{P}_{45}^{17} \mathcal{P}_{58}^{17}=\frac{z_{17} z_{28} z_{36} z_{58}}{z_{12} z_{14} z_{15}^{2} z_{16} z_{23}^{2} z_{24} z_{26} z_{34} z_{37} z_{38} z_{48} z_{56} z_{57}^{2} z_{68}^{2} z_{78}^{2}}$ & 2 \\
\hline $\mathcal{I}^{[4]} \mathcal{P}_{58}^{12} \mathcal{P}_{45}^{17} \mathcal{P}_{58}^{17}=\frac{z_{17} z_{28} z_{36} z_{58}}{z_{14} z_{15}^{2} z_{16}^{2} z_{23}^{2} z_{24} z_{25} z_{26} z_{34} z_{37} z_{38} z_{48} z_{57}^{2} z_{68}^{2} z_{78}^{2}}$ & 3 \\
\hline $\mathcal{I}^{[4]} \mathcal{P}_{58}^{12} \mathcal{P}_{58}^{16} \mathcal{P}_{45}^{17}=\frac{z_{28} z_{36} z_{58}}{z_{14} z_{15}^{2} z_{16} z_{23}^{2} z_{24} z_{25} z_{26} z_{34} z_{37} z_{38} z_{48} z_{56} z_{57} z_{68}^{2} z_{78}^{2}}$ & 0 \\
\hline $\mathcal{I}^{[4]} \mathcal{P}_{45}^{16} \mathcal{P}_{58}^{16} \mathcal{P}_{58}^{17}=\frac{z_{28} z_{36} z_{58}}{z_{12} z_{14} z_{15}^{2} z_{23}^{2} z_{24} z_{26} z_{34} z_{37} z_{38} z_{48} z_{56}^{2} z_{57} z_{68}^{2} z_{78}^{2}}$ & 2 \\
\hline $\mathcal{I}^{[4]} \mathcal{P}_{58}^{12} \mathcal{P}_{45}^{16} \mathcal{P}_{58}^{17}=\frac{z_{28} z_{36} z_{58}}{z_{14} z_{15}^{2} z_{16} z_{23}^{2} z_{24} z_{25} z_{26} z_{34} z_{37} z_{38} z_{48} z_{56} z_{57} z_{68}^{2} z_{78}^{2}}$ & 0 \\
\hline $\mathcal{I}^{[4]} \mathcal{P}_{58}^{12} \mathcal{P}_{45}^{16} \mathcal{P}_{58}^{16}=\frac{z_{28} z_{36} z_{58}}{z_{14} z_{15}^{2} z_{17} z_{23}^{2} z_{24} z_{25} z_{26} z_{34} z_{37} z_{38} z_{48} z_{56}^{2} z_{68}^{2} z_{78}^{2}}$ & 2 \\
\hline $\mathcal{I}^{[4]} \mathcal{P}_{45}^{16} \mathcal{P}_{45}^{17} \mathcal{P}_{58}^{17}=\frac{z_{17} z_{18} z_{28} z_{36} z_{45}}{z_{12} z_{14}^{2} z_{15}^{2} z_{16} z_{23}^{2} z_{24} z_{26} z_{34} z_{37} z_{38} z_{48} z_{56} z_{57}^{2} z_{68}^{2} z_{78}^{2}}$ & 5 \\
\hline $\mathcal{I}^{[4]} \mathcal{P}_{45}^{16} \mathcal{P}_{58}^{16} \mathcal{P}_{45}^{17}=\frac{z_{18} z_{28} z_{36} z_{45}}{z_{12} z_{14}^{2} z_{15}^{2} z_{23}^{2} z_{24} z_{26} z_{34} z_{37} z_{38} z_{48} z_{56}^{2} z_{57} z_{68}^{2} z_{78}^{2}}$ & 4 \\
\hline $\mathcal{I}^{[4]} \mathcal{P}_{58}^{12} \mathcal{P}_{45}^{16} \mathcal{P}_{45}^{17}=\frac{z_{18} z_{28} z_{36} z_{45}}{z_{14}^{2} z_{15}^{2} z_{16} z_{23}^{2} z_{24} z_{25} z_{26} z_{34} z_{37} z_{38} z_{48} z_{56} z_{57} z_{68}^{2} z_{78}^{2}}$ & 2 \\
\hline $\mathcal{I}^{[4]} \mathcal{P}_{45}^{12} \mathcal{P}_{58}^{16} \mathcal{P}_{58}^{17}=\frac{z_{28} z_{36} z_{58}}{z_{14} z_{15}^{2} z_{16} z_{23}^{2} z_{24} z_{25} z_{26} z_{34} z_{37} z_{38} z_{48} z_{56} z_{57} z_{68}^{2} z_{78}^{2}}$ & 0 \\
\hline $\mathcal{I}^{[4]} \mathcal{P}_{45}^{12} \mathcal{P}_{58}^{12} \mathcal{P}_{58}^{17}=\frac{z_{12} z_{28} z_{36} z_{58}}{z_{14} z_{15}^{2} z_{16}^{2} z_{23}^{2} z_{24} z_{25}^{2} z_{26} z_{34} z_{37} z_{38} z_{48} z_{57} z_{68}^{2} z_{78}^{2}}$ & 5 \\
\hline $\mathcal{I}^{[4]} \mathcal{P}_{45}^{12} \mathcal{P}_{58}^{12} \mathcal{P}_{58}^{16}=\frac{z_{12} z_{28} z_{36} z_{58}}{z_{14} z_{15}^{2} z_{16} z_{17} z_{23}^{2} z_{24}^{2} z_{25}^{2} z_{26} z_{34} z_{37} z_{38} z_{48} z_{56} z_{68}^{2} z_{78}^{2}}$ & 4 \\
\hline $\mathcal{I}^{[4]} \mathcal{P}_{45}^{12} \mathcal{P}_{45}^{17} \mathcal{P}_{58}^{17}=\frac{z_{17} z_{18} z_{28} z_{36} z_{45}}{z_{14}^{2} z_{15}^{2} z_{16}^{2} z_{23}^{2} z_{24} z_{25} z_{26} z_{34} z_{37} z_{38} z_{48} z_{57}^{2} z_{68}^{2} z_{78}^{2}}$ & 7 \\
\hline $\mathcal{I}^{[4]} \mathcal{P}_{45}^{12} \mathcal{P}_{58}^{16} \mathcal{P}_{45}^{17}=\frac{z_{18} z_{28} z_{36} z_{45}}{z_{14}^{2} z_{15}^{2} z_{16} z_{23}^{2} z_{24} z_{25} z_{26} z_{34} z_{37} z_{38} z_{48} z_{56} z_{57} z_{68}^{2} z_{78}^{2}}$ & 2 \\
\hline $\mathcal{I}^{[4]} \mathcal{P}_{45}^{12} \mathcal{P}_{58}^{12} \mathcal{P}_{45}^{17}=\frac{z_{12} z_{18} z_{28} z_{36} z_{45}}{z_{14}^{2} z_{15}^{2} z_{16}^{2} z_{23}^{2} z_{24} z_{25}^{2} z_{26} z_{34} z_{37} z_{38} z_{48} z_{57} z_{68}^{2} z_{78}^{2}}$ & 5 \\
\hline $\mathcal{I}^{[4]} \mathcal{P}_{45}^{12} \mathcal{P}_{45}^{16} \mathcal{P}_{58}^{17}=\frac{z_{18} z_{28} z_{36} z_{45}}{z_{14}^{2} z_{15}^{2} z_{16} z_{23}^{2} z_{24} z_{25} z_{26} z_{34} z_{37} z_{38} z_{48} z_{56} z_{57} z_{68}^{2} z_{78}^{2}}$ & 2 \\
\hline $\mathcal{I}^{[4]} \mathcal{P}_{45}^{12} \mathcal{P}_{45}^{16} \mathcal{P}_{58}^{16}=\frac{z_{18} z_{28} z_{36} z_{45}}{z_{14}^{2} z_{15}^{2} z_{17} z_{23}^{2} z_{24} z_{25} z_{26} z_{34} z_{37} z_{38} z_{48} z_{56}^{2} z_{68}^{2} z_{78}^{2}}$ & 5 \\
\hline $\begin{array}{l}\mathcal{I}^{[4]} \mathcal{P}_{45}^{12} \mathcal{P}_{58}^{12} \mathcal{P}_{45}^{16}=\frac{z_{12} z_{18} z_{28} z_{36} z_{45}}{z_{14}^{2} z_{15}^{2} z_{16} z_{17} z_{23}^{2} z_{24} z_{25}^{2} z_{26} z_{34} z_{37} z_{38} z_{48} z_{56} z_{68}^{2} z_{78}^{2}} \\
\mathcal{I}^{[4]} \mathcal{P}_{45}^{12} \mathcal{P}_{45}^{16} \mathcal{P}_{45}^{17}=\frac{z_{18}^{2} z_{28} z_{36} z_{45}^{2}}{z_{14}^{3} z_{15}^{2} z_{16} z_{23}^{2} z_{24} z_{25} z_{26} z_{34} z_{37} z_{38} z_{48} z_{56} z_{57} z_{58} z_{68}^{2} z}\end{array}$ & $\begin{array}{l}4 \\
6\end{array}$ \\
\hline
\end{tabular}

Figure 36. All the three combinations of (3.37).

\begin{tabular}{|lc|}
\hline $\mathcal{I}$ & \# of kept incompatible poles \\
\hline $\mathcal{I}^{[4]} \mathcal{P}_{58}^{12} \mathcal{P}_{58}^{16} \mathcal{P}_{45}^{17}=\frac{z_{28} z_{36} z_{58}}{z_{14} z_{15}^{2} z_{16} z_{23}^{2} z_{24} z_{25} z_{26} z_{34} z_{37} z_{38} z_{48} z_{56} z_{57} z_{68}^{2} z_{78}^{2}}$ & 0 \\
$\mathcal{I}^{[4]} \mathcal{P}_{58}^{12} \mathcal{P}_{45}^{16} \mathcal{P}_{58}^{17}=\frac{z_{28} z_{36} z_{58}}{z_{14} z_{15}^{2} z_{16} z_{23}^{2} z_{24} z_{25} z_{26} z_{34} z_{37} z_{38} z_{48} z_{56} z_{57} z_{68}^{2} z_{78}^{2}}$ & 0 \\
$\mathcal{I}^{[4]} \mathcal{P}_{45}^{12} \mathcal{P}_{58}^{16} \mathcal{P}_{58}^{17}=\frac{z_{28} z_{36} z_{58}}{z_{14} z_{15}^{2} z_{16} z_{23}^{2} z_{24} z_{25} z_{26} z_{34} z_{37} z_{38} z_{48} z_{56} z_{57} z_{68}^{2} z_{78}^{2}}$ & 0 \\
\hline
\end{tabular}

Figure 37. All the combinations without add new poles of (3.37). 

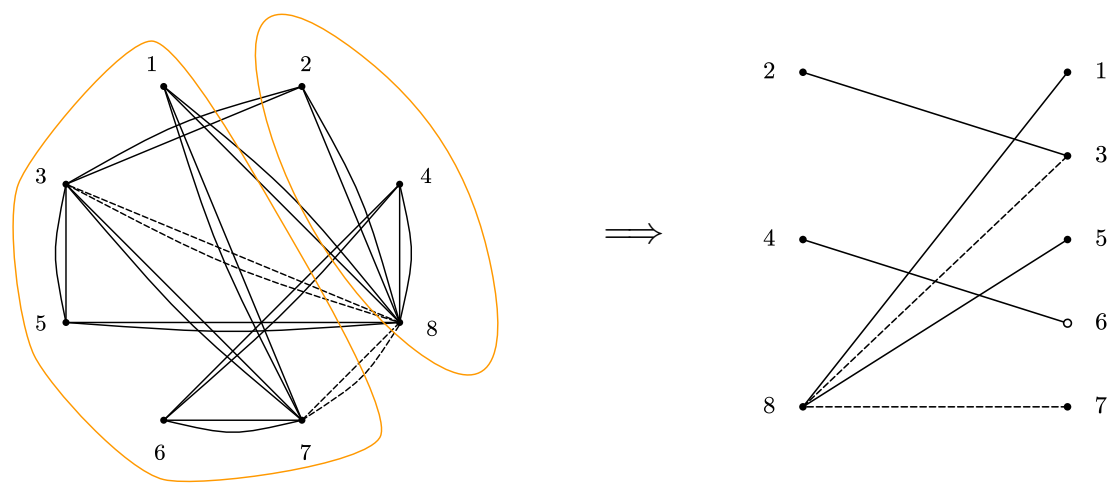

Figure 38. The partition of the CHY-integrand $\frac{z_{38}^{2} z_{78}^{2}}{z_{17}^{2} z_{18}^{2} z_{23}^{2} z_{28}^{2} z_{35}^{2} z_{37}^{2} z_{46}^{2} z_{48}^{2} z_{58}^{2} z_{67}^{2}}$.

which will contain 270 cubic Feynman diagrams (it can be studied using the effective Feynman diagram in the first part of the paper). Following poles have appeared in the final result

$$
\begin{gathered}
\left\{s_{17}, s_{18}, s_{23}, s_{28}, s_{35}, s_{37}, s_{46}, s_{48}, s_{58}, s_{67}, s_{128}, s_{137}, s_{148},\right. \\
s_{158}, s_{167}, s_{235}, s_{237}, s_{248}, s_{258}, s_{357}, s_{367}, s_{458}, s_{467}, s_{468}, \\
\left.s_{1237}, s_{1248}, s_{1258}, s_{1357}, s_{1367}, s_{1458}, s_{1467}, s_{1468}\right\}
\end{gathered}
$$

Let us pick the pole $s_{248}$ which appears in 36 terms. From the figure 38, the linking between the subset $\{2,4,8\}$ and its complement $\{1,3,5,6,7\}$ are solid lines $\{1,8\},\{2,3\}$, $\{4,6\},\{5,8\}$ and the dashed lines $\{3,8\},\{7,8\}$.

Following the algorithm, let us construct the collection (I) with following pure primary cross-ratios

$$
\begin{array}{ll}
\mathcal{P}_{23}^{18}=\frac{z_{18} z_{23}}{z_{12} z_{38}} & \mathcal{P}_{46}^{18}=\frac{z_{18} z_{46}}{z_{14} z_{68}} \quad \mathcal{P}_{46}^{23}=\frac{z_{23} z_{46}}{z_{26} z_{34}} \\
\mathcal{P}_{58}^{23}=\frac{z_{23} z_{58}}{z_{25} z_{38}} & \mathcal{P}_{58}^{46}=\frac{z_{46} z_{58}}{z_{45} z_{68}}
\end{array}
$$

Multiplying all of them together, we will get $\frac{z_{23} z_{46} z_{78}^{2}}{z_{12} z_{14} z_{17}^{2} z_{25} z_{26} z_{28}^{2} z_{34} z_{35}^{2} z_{37}^{2} z_{45} z_{48}^{2} z_{67}^{2} z_{68}^{2}}$, which will produce new pole $s_{68}$ when multiplying the original CHY-integrand. Next, we need to remove the pure primary cross-ratio one by one, until there are non new added poles. However, for all these combinations satisfying the Criterion I, they do not satisfy the Criterion II. We have presented them in figure 39 and listed the number of kept incompatible poles.

Since we can not find the right pick-factor from just the collection (I). According to our algorithm we need to included the collection (III) with following 9 primary cross-ratio factors $^{13}$

$$
\begin{aligned}
& a_{1} \equiv \mathcal{P}_{78 ; 23}^{18}=\frac{z_{18} z_{27}}{z_{12} z_{78}} \quad a_{2} \equiv \mathcal{P}_{38 ; 46}^{23}=\frac{z_{23} z_{68}}{z_{26} z_{38}} \quad a_{3} \equiv \mathcal{P}_{78 ; 23}^{46}=\frac{z_{27} z_{38} z_{46}}{z_{26} z_{34} z_{78}} \\
& a_{4} \equiv \mathcal{P}_{78 ; 46}^{23}=\frac{z_{23} z_{47} z_{68}}{z_{26} z_{34} z_{78}} \quad a_{5} \equiv \mathcal{P}_{78 ; 23}^{58}=\frac{z_{27} z_{58}}{z_{25} z_{78}} \quad a_{6} \equiv \mathcal{P}_{38 ; 46}^{18}=\frac{z_{18} z_{34}}{z_{14} z_{38}} \\
& a_{7} \equiv \mathcal{P}_{78 ; 46}^{18}=\frac{z_{18} z_{47}}{z_{14} z_{78}} \quad a_{8} \equiv \mathcal{P}_{38 ; 46}^{58}=\frac{z_{34} z_{58}}{z_{38} z_{45}} \quad a_{9} \equiv \mathcal{P}_{78 ; 46}^{58}=\frac{z_{47} z_{58}}{z_{45} z_{78}}
\end{aligned}
$$

\footnotetext{
${ }^{13}$ It is worth to recall that according to our construction in (3.6), the $\mathcal{P}_{78 ; 18}^{23}$ is not allowed.
} 


\begin{tabular}{|c|c|}
\hline $\mathcal{I}$ & \# of kept incompatible poles \\
\hline $\mathcal{I}_{8} \mathcal{T}_{1 ; 1} \equiv \mathcal{I}_{8} \mathcal{P}_{23}^{18}=\frac{z_{38} z_{78}^{2}}{z_{12} z_{17}^{2} z_{18} z_{23} z_{28}^{2} z_{35}^{2} z_{37}^{2} z_{46}^{2} z_{48}^{2} z_{58}^{2} z_{67}^{2}}$ & 10 \\
\hline $\mathcal{I}_{8} \mathcal{T}_{1 ; 2} \equiv \mathcal{I}_{8} \mathcal{P}_{46}^{23}=\frac{z_{38}^{2} z_{78}^{2}}{z_{17}^{2} z_{18}^{2} z_{23} z_{26} z_{28}^{2} z_{34} z_{35}^{2} z_{37}^{2} z_{46} z_{48}^{2} z_{58}^{2} z_{67}^{2}}$ & 9 \\
\hline $\mathcal{I}_{8} \mathcal{T}_{1 ; 3} \equiv \mathcal{I}_{8} \mathcal{P}_{58}^{23}=\frac{z_{38} z_{78}^{2}}{z_{17}^{2} z_{18}^{2} z_{23} z_{25} z_{28}^{2} z_{35}^{2} z_{37}^{2} z_{46}^{2} z_{48}^{2} z_{58} z_{67}^{2}}$ & 11 \\
\hline $\mathcal{I}_{8} \mathcal{T}_{1 ; 4} \equiv \mathcal{I}_{8} \mathcal{P}_{46}^{18}=\frac{z_{38}^{2} z_{78}^{2}}{z_{14} z_{17}^{2} z_{18} z_{23}^{2} z_{28}^{2} z_{35}^{2} z_{37}^{7} z_{46} z_{48}^{2} z_{58}^{2} z_{67}^{2} z_{68}}$ & 12 \\
\hline $\mathcal{I}_{8} \mathcal{T}_{1 ; 5} \equiv \mathcal{I}_{8} \mathcal{P}_{58}^{46}=\frac{z_{38}^{2} z_{78}^{2}}{z_{17}^{2} z_{18}^{2} z_{23}^{2} z_{28}^{2} z_{35}^{2} z_{37}^{2} z_{45} z_{46} z_{48}^{2} z_{58} z_{67}^{2} z_{68}}$ & 11 \\
\hline $\mathcal{I}_{8} \mathcal{T}_{2 ; 1} \equiv \mathcal{I}_{8} \mathcal{P}_{23}^{18} \mathcal{P}_{58}^{23}=\frac{z_{78}^{2}}{z_{12} z_{17}^{2} z_{18} z_{25} z_{28}^{2} z_{35}^{2} z_{37}^{2} z_{46}^{2} z_{48}^{2} z_{58} z_{67}^{2}}$ & 7 \\
\hline $\mathcal{I}_{8} \mathcal{T}_{2 ; 2} \equiv \mathcal{I}_{8} \mathcal{P}_{23}^{18} \mathcal{P}_{46}^{18}=\frac{z_{38} z_{78}^{2}}{z_{12} z_{14} z_{17}^{2} z_{23} z_{28}^{2} z_{35}^{2} z_{37}^{2} z_{46} z_{48}^{2} z_{58}^{2} z_{67}^{2} z_{68}}$ & 6 \\
\hline $\mathcal{I}_{8} \mathcal{T}_{2 ; 3} \equiv \mathcal{I}_{8} \mathcal{P}_{23}^{18} \mathcal{P}_{58}^{46}=\frac{z_{38} z_{78}^{2}}{z_{12} z_{17}^{2} z_{18} z_{23} z_{28}^{2} z_{35}^{2} z_{37}^{2} z_{45} z_{46} z_{48}^{2} z_{58} z_{67}^{2} z_{68}}$ & 4 \\
\hline $\mathcal{I}_{8} \mathcal{T}_{2 ; 4} \equiv \mathcal{I}_{8} \mathcal{P}_{46}^{23} \mathcal{P}_{58}^{23}=\frac{z_{38} z_{78}^{2}}{z_{17}^{2} z_{18}^{2} z_{25} z_{26} z_{28}^{2} z_{34} z_{35}^{2} z_{37}^{2} z_{46} z_{48}^{2} z_{58} z_{67}^{2}}$ & 5 \\
\hline $\mathcal{I}_{8} \mathcal{T}_{2 ; 5} \equiv \mathcal{I}_{8} \mathcal{P}_{46}^{18} \mathcal{P}_{58}^{23}=\frac{z_{38} z_{78}^{2}}{z_{14} z_{17}^{2} z_{18} z_{23} z_{25} z_{28}^{2} z_{35}^{2} z_{37}^{2} z_{46} z_{48}^{2} z_{58} z_{67}^{2} z_{68}}$ & 6 \\
\hline $\mathcal{I}_{8} \mathcal{T}_{2 ; 6} \equiv \mathcal{I}_{8} \mathcal{P}_{58}^{23} \mathcal{P}_{58}^{46}=\frac{z_{38} z_{78}^{2}}{z_{17}^{2} z_{18}^{2} z_{23} z_{25} z_{28}^{2} z_{35}^{2} z_{37}^{2} z_{45} z_{46} z_{48}^{2} z_{67}^{2} z_{68}}$ & 6 \\
\hline $\mathcal{I}_{8} \mathcal{T}_{3 ; 1} \equiv \mathcal{I}_{8} \mathcal{P}_{23}^{18} \mathcal{P}_{46}^{18} \mathcal{P}_{58}^{23}=\frac{z_{78}^{2}}{z_{12} z_{14} z_{17}^{2} z_{25} z_{28}^{2} z_{35}^{2} z_{37}^{2} z_{46} z_{48}^{2} z_{58} z_{67}^{2} z_{68}}$ & 3 \\
\hline $\mathcal{I}_{8} \mathcal{T}_{3 ; 2} \equiv \mathcal{I}_{8} \mathcal{P}_{23}^{18} \mathcal{P}_{58}^{23} \mathcal{P}_{58}^{46}=\frac{z_{78}^{2}}{z_{12} z_{17}^{2} z_{18} z_{25} z_{28}^{2} z_{35}^{2} z_{37}^{2} z_{45} z_{46} z_{48}^{2} z_{66}^{2} z_{68}}$ & 2 \\
\hline
\end{tabular}

Figure 39. All the combinations without add new poles of (3.39).

There are 13's $\mathcal{T}_{i ; k}$ according to (3.7) with $i=3,2,1$ and $N_{3}=2, N_{2}=6, N_{1}=5$ respectively. Searching along the nested loops, we find that

- (1) With $m=1$, we find all $13 \times 9$ can not satisfy both Criterion I and II at the same time.

- (2) With $m=2$, we find all $13 \times 36$ can not satisfy both Criterion I and II at the same time.

- (3) With $m=3$, we tested all $13 \times 84$ items. Twelve combinations satisfy both Criterion I and II:

$$
\begin{array}{lll}
\mathcal{T}_{2 ; 1}: & \mathcal{P}_{23}^{18} \mathcal{P}_{58}^{23} \mathcal{P}_{38 ; 46}^{18} \mathcal{P}_{78 ; 23}^{46} \mathcal{P}_{78 ; 46}^{58} & \mathcal{P}_{23}^{18} \mathcal{P}_{58}^{23} \mathcal{P}_{78 ; 46}^{18} \mathcal{P}_{78 ; 23}^{46} \mathcal{P}_{38 ; 46}^{58} \\
\mathcal{T}_{2 ; 2}: & \mathcal{P}_{23}^{18} \mathcal{P}_{46}^{18} \mathcal{P}_{38 ; 46}^{23} \mathcal{P}_{78 ; 23}^{58} \mathcal{P}_{78 ; 46}^{58} & \mathcal{P}_{23}^{18} \mathcal{P}_{46}^{18} \mathcal{P}_{78 ; 46}^{23} \mathcal{P}_{38 ; 46}^{58} \mathcal{P}_{78 ; 23}^{58} \\
\mathcal{T}_{2 ; 3}: & \mathcal{P}_{23}^{18} \mathcal{P}_{58}^{46} \mathcal{P}_{78 ; 46}^{18} \mathcal{P}_{38 ; 46}^{23} \mathcal{P}_{78 ; 23}^{58} & \mathcal{P}_{23}^{18} \mathcal{P}_{58}^{46} \mathcal{P}_{38 ; 46}^{18} \mathcal{P}_{78 ; 46}^{23} \mathcal{P}_{78 ; 23}^{58} \\
\mathcal{T}_{2 ; 4}: & \mathcal{P}_{46}^{23} \mathcal{P}_{58}^{23} \mathcal{P}_{38 ; 46}^{18} \mathcal{P}_{78 ; 23}^{18} \mathcal{P}_{78 ; 46}^{58} & \mathcal{P}_{46}^{23} \mathcal{P}_{58}^{23} \mathcal{P}_{78 ; 23}^{18} \mathcal{P}_{78 ; 46}^{18} \mathcal{P}_{38 ; 46}^{58} \\
\mathcal{T}_{2 ; 5}: & \mathcal{P}_{46}^{18} \mathcal{P}_{58}^{23} \mathcal{P}_{78 ; 23}^{18} \mathcal{P}_{38 ; 46}^{23} \mathcal{P}_{78 ; 46}^{58} & \mathcal{P}_{46}^{18} \mathcal{P}_{58}^{23} \mathcal{P}_{78 ; 23}^{18} \mathcal{P}_{78 ; 46}^{23} \mathcal{P}_{38 ; 46}^{58} \\
\mathcal{T}_{2 ; 6}: & \mathcal{P}_{58}^{23} \mathcal{P}_{58}^{46} \mathcal{P}_{78 ; 23}^{18} \mathcal{P}_{78 ; 46}^{18} \mathcal{P}_{38 ; 46}^{23} & \mathcal{P}_{58}^{23} \mathcal{P}_{58}^{46} \mathcal{P}_{38 ; 46}^{18} \mathcal{P}_{78 ; 23}^{18} \mathcal{P}_{78 ; 46}^{23}
\end{array}
$$


All of above twelve combinations of primary cross-ratio factors give the same pickfactor:

$$
\frac{z_{18}^{2} z_{23}^{2} z_{27} z_{46} z_{47} z_{58}^{2}}{z_{12} z_{14} z_{25} z_{26} z_{38}^{2} z_{45} z_{78}^{2}}
$$

It gives a new integrand.

$$
\mathcal{I}_{8}^{\prime}=\frac{z_{27} z_{47}}{z_{12} z_{14} z_{17}^{2} z_{25} z_{26} z_{28}^{2} z_{35}^{2} z_{37}^{2} z_{45} z_{46} z_{48}^{2} z_{67}^{2}}
$$

The Feynman diagrams corresponding to this new integrand contains the only $s_{248}$ terms.

$$
\begin{aligned}
& -\frac{1}{s_{17} s_{35} s_{48} s_{167} s_{248}}-\frac{1}{s_{28} s_{35} s_{67} s_{167} s_{248}}-\frac{1}{s_{35} s_{48} s_{67} s_{167} s_{248}}-\frac{1}{s_{17} s_{28} s_{35} s_{167} s_{248}} \\
& -\frac{1}{s_{35} s_{48} s_{67} s_{248} s_{1248}}-\frac{1}{s_{28} s_{35} s_{248} s_{357} s_{1248}}-\frac{1}{s_{28} s_{37} s_{248} s_{357} s_{1248}}-\frac{1}{s_{28} s_{35} s_{67} s_{248} s_{1248}} \\
& -\frac{1}{s_{48} s_{67} s_{248} s_{367} s_{1248}}-\frac{1}{s_{17} s_{28} s_{35} s_{248} s_{1357}}-\frac{1}{s_{17} s_{35} s_{48} s_{248} s_{1357}}-\frac{1}{s_{28} s_{67} s_{248} s_{367} s_{1248}} \\
& -\frac{1}{s_{37} s_{48} s_{248} s_{357} s_{1248}}-\frac{1}{s_{28} s_{37} s_{248} s_{367} s_{1248}}-\frac{1}{s_{37} s_{48} s_{248} s_{367} s_{1248}}-\frac{1}{s_{35} s_{48} s_{248} s_{357} s_{1248}} \\
& -\frac{1}{s_{28} s_{37} s_{137} s_{248} s_{1357}}-\frac{1}{s_{17} s_{48} s_{137} s_{248} s_{1357}}-\frac{1}{s_{37} s_{48} s_{137} s_{248} s_{1357}}-\frac{1}{s_{17} s_{28} s_{137} s_{248} s_{1357}} \\
& -\frac{1}{s_{28} s_{37} s_{248} s_{357} s_{1357}}-\frac{1}{s_{35} s_{48} s_{248} s_{357} s_{1357}}-\frac{1}{s_{37} s_{48} s_{248} s_{357} s_{1357}}-\frac{1}{s_{28} s_{35} s_{248} s_{357} s_{1357}} \\
& -\frac{1}{s_{28} s_{37} s_{137} s_{248} s_{1367}}-\frac{1}{s_{17} s_{48} s_{137} s_{248} s_{1367}}-\frac{1}{s_{37} s_{48} s_{137} s_{248} s_{1367}}-\frac{1}{s_{17} s_{28} s_{137} s_{248} s_{1367}} \\
& -\frac{1}{s_{17} s_{48} s_{167} s_{248} s_{1367}}-\frac{1}{s_{28} s_{67} s_{167} s_{248} s_{1367}}-\frac{1}{s_{48} s_{67} s_{167} s_{248} s_{1367}}-\frac{1}{s_{17} s_{28} s_{167} s_{248} s_{1367}} \\
& -\frac{1}{s_{37} s_{48} s_{248} s_{367} s_{1367}}-\frac{1}{s_{28} s_{67} s_{248} s_{367} s_{1367}}-\frac{1}{s_{48} s_{67} s_{248} s_{367} s_{1367}}-\frac{1}{s_{28} s_{37} s_{248} s_{367} s_{1367}}
\end{aligned}
$$

- (4) Although we could stop our algorithm with $m=3$. For this simple example, we verified the case with $m>3$ for completeness. We find that for $m>3$, there do not exist any combinations that satisfy both Criterion I and II at the same time.

The second example is more plentiful, the CHY-integrand

$$
\mathcal{I}_{8}^{[2]}=\frac{z_{68}^{2}}{z_{13}^{2} z_{16} z_{17} z_{24}^{2} z_{28}^{2} z_{35} z_{36} z_{46}^{2} z_{56} z_{58}^{2} z_{67} z_{78}^{2}}
$$


which will contain 28 cubic Feynman diagrams:

$$
\begin{aligned}
& +\frac{1}{s_{13} s_{24} s_{78} s_{136} s_{578}}+\frac{1}{s_{13} s_{24} s_{58} s_{246} s_{578}}+\frac{1}{s_{13} s_{46} s_{58} s_{246} s_{578}}+\frac{1}{s_{13} s_{24} s_{78} s_{246} s_{578}} \\
& +\frac{1}{s_{13} s_{46} s_{78} s_{246} s_{578}}+\frac{1}{s_{13} s_{28} s_{46} s_{258} s_{1346}}+\frac{1}{s_{13} s_{46} s_{58} s_{258} s_{1346}}+\frac{1}{s_{13} s_{28} s_{136} s_{258} s_{1346}} \\
& +\frac{1}{s_{13} s_{58} s_{136} s_{258} s_{1346}}+\frac{1}{s_{13} s_{28} s_{46} s_{278} s_{1346}}+\frac{1}{s_{13} s_{46} s_{78} s_{278} s_{1346}}+\frac{1}{s_{13} s_{28} s_{136} s_{278} s_{1346}} \\
& +\frac{1}{s_{13} s_{78} s_{136} s_{278} s_{1346}}+\frac{1}{s_{13} s_{46} s_{58} s_{578} s_{1346}}+\frac{1}{s_{13} s_{46} s_{78} s_{578} s_{1346}}+\frac{1}{s_{13} s_{58} s_{136} s_{578} s_{1346}} \\
& +\frac{1}{s_{13} s_{78} s_{136} s_{578} s_{1346}}+\frac{1}{s_{13} s_{24} s_{78} s_{136} s_{1356}}+\frac{1}{s_{13} s_{24} s_{136} s_{248} s_{1356}}+\frac{1}{s_{13} s_{28} s_{136} s_{248} s_{1356}} \\
& +\frac{1}{s_{13} s_{28} s_{136} s_{278} s_{1356}}+\frac{1}{s_{13} s_{78} s_{136} s_{278} s_{1356}}+\frac{1}{s_{13} s_{24} s_{58} s_{136} s_{1367}}+\frac{1}{s_{13} s_{24} s_{136} s_{248} s_{1367}} \\
& +\frac{1}{s_{13} s_{28} s_{136} s_{248} s_{1367}}+\frac{1}{s_{13} s_{28} s_{136} s_{258} s_{1367}}+\frac{1}{s_{13} s_{58} s_{136} s_{258} s_{1367}}+\frac{1}{s_{13} s_{24} s_{58} s_{136} s_{578}}
\end{aligned}
$$

Let us pick the pole $s_{258}$ which appears in 6 terms. The linking between the subset $\{2,5,8\}$ and its complement $\{1,3,4,6,7\}$ are containing solid lines $\{2,4\},\{5,3\},\{5,6\}$ and the dashed lines $\{8,6\}$. Following the algorithm, let us construct the collection (I) with following pure primary cross-ratios

$$
\begin{aligned}
& \mathcal{P}_{35}^{24}=\frac{z_{24} z_{35}}{z_{23} z_{45}} \quad \mathcal{P}_{56}^{24}=\frac{z_{24} z_{56}}{z_{26} z_{45}} \quad \mathcal{P}_{78}^{24}=\frac{z_{24} z_{78}}{z_{27} z_{48}} \\
& \mathcal{P}_{78}^{35}=\frac{z_{35} z_{78}}{z_{38} z_{57}} \quad \mathcal{P}_{78}^{56}=\frac{z_{56} z_{78}}{z_{57} z_{68}}
\end{aligned}
$$

Multiplying all of them together, we will get $\frac{z_{24}^{3} z_{35}^{2} z_{56}^{2} z_{78}^{3}}{z_{23} z_{26} z_{27} z_{38} z_{45}^{2} z_{48} z_{57}^{2} z_{68}}$, which will produce new pole $s_{57}, s_{45}$ when multiplying the original $\mathcal{I}_{8}^{[2]}$. We need to remove the pure primary crossratio until there are non new added poles as before. We have presented all combinations that satisfying the Criterion I, but do not satisfy the Criterion II in figure 40 and label the number of kept incompatible poles. Since they all kept incompatible poles, we can not find the right pick-factor from just the collection (I). According to our algorithm we need to included the collection (III) with following five primary cross-ratio factors

$$
\begin{aligned}
a_{1} & \equiv \mathcal{P}_{68 ; 24}^{35}=\frac{z_{26} z_{35} z_{48}}{z_{23} z_{45} z_{68}} \quad a_{2} \equiv \mathcal{P}_{68 ; 35}^{24}=\frac{z_{24} z_{38} z_{56}}{z_{23} z_{45} z_{68}} \quad a_{3} \equiv \mathcal{P}_{68 ; 24}^{56}=\frac{z_{48} z_{56}}{z_{45} z_{68}} \\
a_{4} & \equiv \mathcal{P}_{68 ; 24}^{78}=\frac{z_{26} z_{78}}{z_{27} z_{68}} \quad a_{5} \equiv \mathcal{P}_{68 ; 35}^{78}=\frac{z_{56} z_{78}}{z_{57} z_{68}}
\end{aligned}
$$

There are 9's $\mathcal{T}_{i ; k}$ according to (3.7) with $i=2,1$ and $N_{2}=5, N_{1}=4$ respectively. Searching along the nested loops, we find that

- (1) With $m=1$, we search all $9 \times 5$ combinations, five of them satisfy both Criterion I and II: 


\begin{tabular}{|ll|}
\hline $\mathcal{I}$ & \# of kept incompatible poles \\
\hline $\mathcal{I}_{8}^{[2]} \mathcal{T}_{2 ; 1} \equiv \mathcal{I}_{8}^{[2]} \mathcal{P}_{78}^{24} \mathcal{P}_{78}^{56}=\frac{z_{68}}{z_{13}^{2} z_{16} z_{17} z_{24} z_{27} z_{28}^{2} z_{35} z_{36} z_{46}^{2} z_{48} z_{57} z_{58}^{2} z_{67}}$ & 1 \\
$\mathcal{I}_{8}^{[2]} \mathcal{T}_{2 ; 2} \equiv \mathcal{I}_{8}^{[2]} \mathcal{P}_{78}^{24} \mathcal{P}_{78}^{35}=\frac{z_{68}^{2}}{z_{13}^{2} z_{16} z_{17} z_{24} z_{27} z_{28}^{2} z_{36} z_{38} z_{46}^{2} z_{48} z_{56} z_{57} z_{58}^{2} z_{67}}$ & 1 \\
$\mathcal{I}_{8}^{[2]} \mathcal{T}_{2 ; 3} \equiv \mathcal{I}_{8}^{[2]} \mathcal{P}_{56}^{24} \mathcal{P}_{78}^{56}=\frac{z_{56} z_{68}}{z_{13}^{2} z_{16} z_{17} z_{24} z_{26} z_{28}^{2} z_{35} z_{36} z_{45} z_{46}^{2} z_{57} z_{58}^{2} z_{67} z_{78}}$ & 2 \\
$\mathcal{I}_{8}^{[2]} \mathcal{T}_{2 ; 4} \equiv \mathcal{I}_{8}^{[2]} \mathcal{P}_{56}^{24} \mathcal{P}_{78}^{35}=\frac{z_{68}^{2}}{z_{13}^{2} z_{16} z_{17} z_{24} z_{26} z_{28}^{2} z_{36} z_{38} z_{45} z_{46}^{2} z_{57} z_{58}^{2} z_{67} z_{78}}$ & 2 \\
$\mathcal{I}_{8}^{[2]} \mathcal{T}_{2 ; 5} \equiv \mathcal{I}_{8}^{[2]} \mathcal{P}_{35}^{24} \mathcal{P}_{78}^{56}=\frac{z_{68}}{z_{13}^{2} z_{16} z_{17} z_{23} z_{24} z_{28}^{2} z_{36} z_{45} z_{46}^{2} z_{57} z_{58}^{2} z_{67} z_{78}}$ & 1 \\
$\mathcal{I}_{8}^{[2]} \mathcal{T}_{1 ; 1} \equiv \mathcal{I}_{8}^{[2]} \mathcal{P}_{78}^{56}=\frac{z_{68}}{z_{13}^{2} z_{16} z_{17} z_{24}^{2} z_{28}^{2} z_{35} z_{36} z_{46}^{2} z_{57} z_{58}^{2} z_{67} z_{78}}$ & 4 \\
$\mathcal{I}_{8}^{[2]} \mathcal{T}_{1 ; 2} \equiv \mathcal{I}_{8}^{[2]} \mathcal{P}_{78}^{35}=\frac{z_{68}^{2}}{z_{13}^{2} z_{16} z_{17} z_{24}^{2} z_{28}^{2} z_{36} z_{38} z_{46}^{2} z_{56} z_{57} z_{58}^{2} z_{67} z_{78}}$ & 4 \\
$\mathcal{I}_{8}^{[2]} \mathcal{T}_{1 ; 3} \equiv \mathcal{I}_{8}^{[2]} \mathcal{P}_{78}^{24}=\frac{z_{68}^{2}}{z_{13}^{2} z_{16} z_{17} z_{24} z_{27} z_{28}^{2} z_{35} z_{36} z_{46}^{2} z_{48} z_{56} z_{58}^{2} z_{67} z_{78}}$ & 4 \\
$\mathcal{I}_{8}^{[2]} \mathcal{T}_{1 ; 4} \equiv \mathcal{I}_{8}^{[2]} \mathcal{P}_{56}^{24}=\frac{z_{68}^{2}}{z_{13}^{2} z_{16} z_{17} z_{24} z_{26} z_{28}^{2} z_{35} z_{36} z_{45} z_{46}^{2} z_{58}^{2} z_{67} z_{78}^{2}}$ & 4 \\
\hline
\end{tabular}

Figure 40. All the combinations without add new poles of (3.43).

$$
\begin{array}{ll}
\mathcal{T}_{2 ; 1}: & \mathcal{P}_{78}^{24} \mathcal{P}_{78}^{56} \mathcal{P}_{68 ; 24}^{35}=\frac{z_{24} z_{26} z_{35} z_{56} z_{78}^{2}}{z_{23} z_{27} z_{45} z_{57} z_{68}^{2}} \quad \mathcal{P}_{78}^{24} \mathcal{P}_{78}^{56} \mathcal{P}_{68 ; 24}^{56}=\frac{z_{24} z_{56}^{2} z_{78}^{2}}{z_{27} z_{45} z_{57} z_{68}^{2}} \\
\mathcal{T}_{2 ; 2}: & \mathcal{P}_{78}^{24} \mathcal{P}_{78}^{35} \mathcal{P}_{68 ; 24}^{56}=\frac{z_{24} z_{35} z_{56} z_{78}^{2}}{z_{27} z_{38} z_{45} z_{57} z_{68}} \\
\mathcal{T}_{2 ; 3}: & \mathcal{P}_{56}^{24} \mathcal{P}_{78}^{56} \mathcal{P}_{68 ; 24}^{78}=\frac{z_{24} z_{65}^{2} z_{78}^{2}}{z_{27} z_{45} z_{57} z_{68}^{2}} \\
\mathcal{T}_{2 ; 4}: & \mathcal{P}_{56}^{24} \mathcal{P}_{78}^{35} \mathcal{P}_{68 ; 24}^{78}=\frac{z_{24} z_{35} z_{56} z_{78}^{2}}{z_{27} z_{38} z_{45} z_{57} z_{68}} \\
\mathcal{T}_{2 ; 5}: & \mathcal{P}_{35}^{24} \mathcal{P}_{78}^{56} \mathcal{P}_{68 ; 24}^{78}=\frac{z_{24} z_{26} z_{35} z_{56} z_{78}^{2}}{z_{23} z_{27} z_{45} z_{57} z_{68}^{2}}
\end{array}
$$

- (2) With $m=2$, we test all $9 \times 10$ items that four combinations satisfy both Criterion I and II at the same time.

$$
\begin{array}{ll}
\mathcal{T}_{1 ; 1}: & \mathcal{P}_{78}^{56} \mathcal{P}_{68 ; 35}^{24} \mathcal{P}_{68 ; 24}^{78}=\frac{z_{24} z_{26} z_{38} z_{56}^{2} z_{78}^{2}}{z_{23} z_{27} z_{45} z_{57} z_{68}^{3}} \\
\mathcal{T}_{1 ; 2}: & \mathcal{P}_{78}^{35} \mathcal{P}_{68 ; 35}^{24} \mathcal{P}_{68 ; 24}^{78}=\frac{z_{24} z_{26} z_{35} z_{56} z_{78}^{2}}{z_{23} z_{27} z_{45} z_{57} z_{68}^{2}} \\
\mathcal{T}_{2 ; 1}: & \mathcal{P}_{78}^{24} \mathcal{P}_{78}^{56} \mathcal{P}_{68 ; 24}^{35}=\frac{z_{24} z_{26} z_{35} z_{56} z_{78}^{2}}{z_{23} z_{27} z_{45} z_{57} z_{68}^{2}} \quad \mathcal{P}_{78}^{24} \mathcal{P}_{78}^{56} \mathcal{P}_{68 ; 24}^{56}=\frac{z_{24} z_{56}^{2} z_{78}^{2}}{z_{27} z_{45} z_{57} z_{68}^{2}} \\
\mathcal{T}_{2 ; 3}: & \mathcal{P}_{56}^{24} \mathcal{P}_{78}^{56} \mathcal{P}_{68 ; 24}^{78}=\frac{z_{24} z_{56}^{2} z_{78}^{2}}{z_{27} z_{45} z_{57} z_{68}^{2}}
\end{array}
$$

- (3) We verified that for $m>2$, there do not exist any combinations that satisfy both Criterion I and II at the same time. 
All of above combinations of primary cross-ratio factors give four different pick-factors:
(a) $: \frac{z_{24} z_{26} z_{35} z_{56} z_{78}^{2}}{z_{23} z_{27} z_{45} z_{57} z_{68}^{2}}$
(b) : $\frac{z_{24} z_{35} z_{56} z_{78}^{2}}{z_{27} z_{38} z_{45} z_{57} z_{68}}$
(c) $: \frac{z_{24} z_{56}^{2} z_{78}^{2}}{z_{27} z_{45} z_{57} z_{68}^{2}}$
$(d): \frac{z_{24} z_{26} z_{38} z_{56}^{2} z_{78}^{2}}{z_{23} z_{27} z_{45} z_{57} z_{68}^{3}}$

When multiplying each of them to the original CHY-integrand, they all produce the same Feynman diagrams containing the pole $s_{258}$.

$$
\begin{aligned}
& \frac{1}{s_{13} s_{46} s_{58} s_{258} s_{1346}}+\frac{1}{s_{13} s_{28} s_{136} s_{258} s_{1346}}+\frac{1}{s_{13} s_{28} s_{46} s_{258} s_{1346}} \\
& +\frac{1}{s_{13} s_{28} s_{136} s_{258} s_{1367}}+\frac{1}{s_{13} s_{58} s_{136} s_{258} s_{1367}}+\frac{1}{s_{13} s_{58} s_{136} s_{258} s_{1346}}
\end{aligned}
$$

Let us give the third example that requires the collection (III)

$$
\mathcal{I}_{8}^{[3]}=\frac{z_{38}^{2}}{z_{13}^{2} z_{18}^{2} z_{25} z_{26} z_{28}^{2} z_{34} z_{36}^{2} z_{37} z_{45}^{2} z_{47} z_{56} z_{78}^{2}}
$$

which will contain 10 cubic Feynman diagrams:

$$
\begin{aligned}
& +\frac{1}{s_{28} s_{36} s_{45} s_{136} s_{278}}+\frac{1}{s_{13} s_{45} s_{78} s_{136} s_{278}}+\frac{1}{s_{36} s_{45} s_{78} s_{136} s_{278}}+\frac{1}{s_{13} s_{28} s_{45} s_{136} s_{278}} \\
& +\frac{1}{s_{28} s_{36} s_{45} s_{128} s_{1278}}+\frac{1}{s_{18} s_{36} s_{45} s_{178} s_{1278}}+\frac{1}{s_{36} s_{45} s_{78} s_{178} s_{1278}}+\frac{1}{s_{18} s_{36} s_{45} s_{128} s_{1278}} \\
& +\frac{1}{s_{36} s_{45} s_{78} s_{278} s_{1278}}+\frac{1}{s_{28} s_{36} s_{45} s_{278} s_{1278}}
\end{aligned}
$$

Let us pick the pole $s_{1278}$ which appears in 6 terms. From the figure 38 , the linking between the subset $\{1,2,7,8\}$ and its complement $\{3,4,5,6\}$ are solid lines $\{1,3\},\{2,5\}$, $\{2,6\},\{7,3\},\{7,4\}$ and the dashed lines $\{8,3\}$. Following the algorithm, let us construct the collection (I) with following pure primary cross-ratios

$$
\begin{array}{llll}
\mathcal{P}_{25}^{13}=\frac{z_{13} z_{25}}{z_{15} z_{23}} & \mathcal{P}_{26}^{13}=\frac{z_{13} z_{26}}{z_{16} z_{23}} & \mathcal{P}_{47}^{13}=\frac{z_{13} z_{47}}{z_{14} z_{37}} & \mathcal{P}_{37}^{25}=\frac{z_{25} z_{37}}{z_{23} z_{57}} \\
\mathcal{P}_{47}^{25}=\frac{z_{25} z_{47}}{z_{24} z_{57}} & \mathcal{P}_{37}^{26}=\frac{z_{26} z_{37}}{z_{23} z_{67}} & \mathcal{P}_{47}^{26}=\frac{z_{26} z_{47}}{z_{24} z_{67}}
\end{array}
$$

Multiplying all of them together, we will get $\frac{z_{13}^{3} z_{25}^{3} z_{26}^{3} z_{37} z_{47}^{3}}{z_{14} z_{15} z_{16} z_{23}^{4} z_{24}^{2} z_{57}^{2} z_{67}^{2}}$, which will produce new pole $s_{23}, s_{24}, s_{57}, s_{67}$ when multiplying with the original CHY-integrand $\mathcal{I}_{8}^{[3]}$. We have presented combinations satisfying the Criterion I in figure 41 with the number of kept incompatible poles. According to the algorithm, we construct the collection (III) with following 9 primary cross-ratio factors

$$
\begin{aligned}
& a_{1} \equiv \mathcal{P}_{38 ; 25}^{13}=\frac{z_{13} z_{58}}{z_{15} z_{38}} \quad a_{2} \equiv \mathcal{P}_{38 ; 26}^{13}=\frac{z_{13} z_{68}}{z_{16} z_{38}} \quad a_{3} \equiv \mathcal{P}_{38 ; 47}^{13}=\frac{z_{13} z_{48}}{z_{14} z_{38}} \\
& a_{4} \equiv \mathcal{P}_{38 ; 25}^{37}=\frac{z_{37} z_{58}}{z_{38} z_{57}} \quad a_{5} \equiv \mathcal{P}_{38 ; 47}^{25}=\frac{z_{25} z_{37} z_{48}}{z_{24} z_{38} z_{57}} \quad a_{6} \equiv \mathcal{P}_{38 ; 25}^{47}=\frac{z_{23} z_{47} z_{58}}{z_{24} z_{38} z_{57}} \\
& a_{7} \equiv \mathcal{P}_{38 ; 26}^{37}=\frac{z_{37} z_{68}}{z_{38} z_{67}} \quad a_{8} \equiv \mathcal{P}_{38 ; 47}^{26}=\frac{z_{26} z_{37} z_{48}}{z_{24} z_{38} z_{67}} \quad a_{9} \equiv \mathcal{P}_{38 ; 26}^{47}=\frac{z_{23} z_{47} z_{68}}{z_{24} z_{38} z_{67}}
\end{aligned}
$$

There are 6 's $\mathcal{T}_{i ; k}$ according to (3.7) with $i=2,1$ and $N_{2}=3, N_{1}=3$ respectively. Searching along the nested loops, we find that 


\begin{tabular}{|lc|}
\hline $\mathcal{I}$ & \# of kept incompatible poles \\
\hline $\mathcal{I}_{8}^{[3]} \mathcal{T}_{2 ; 1} \equiv \mathcal{I}_{8}^{[3]} \mathcal{P}_{47}^{25} \mathcal{P}_{37}^{26}=\frac{z_{38}^{2}}{z_{13}^{2} z_{18}^{2} z_{23} z_{24} z_{28}^{2} z_{34} z_{36}^{2} z_{45}^{2} z_{56} z_{57} z_{67} z_{78}^{2}}$ & 2 \\
$\mathcal{I}_{8}^{[3]} \mathcal{T}_{2 ; 2} \equiv \mathcal{I}_{8}^{[3]} \mathcal{P}_{37}^{25} \mathcal{P}_{47}^{26}=\frac{z_{38}^{2}}{z_{13}^{2} z_{18}^{2} z_{23} z_{24} z_{28}^{2} z_{34} z_{36}^{2} z_{45}^{2} z_{56} z_{57} z_{67} z_{78}^{2}}$ & 1 \\
$\mathcal{I}_{8}^{[3]} \mathcal{T}_{2 ; 3} \equiv \mathcal{I}_{8}^{[3]} \mathcal{P}_{26}^{13} \mathcal{P}_{47}^{25}=\frac{z_{38}^{2}}{z_{13} z_{16} z_{18}^{2} z_{23} z_{24} z_{28}^{2} z_{34} z_{36}^{2} z_{37} z_{45}^{2} z_{56} z_{57} z_{78}^{2}}$ & 2 \\
$\mathcal{I}_{8}^{[3]} \mathcal{T}_{1 ; 1} \equiv \mathcal{I}_{8}^{[3]} \mathcal{P}_{37}^{26}=\frac{z_{38}^{2}}{z_{13}^{2} z_{18}^{2} z_{23} z_{25} z_{28}^{2} z_{34} z_{36}^{2} z_{45}^{2} z_{47} z_{56} z_{67} z_{78}^{2}}$ & 2 \\
$\mathcal{I}_{8}^{[3]} \mathcal{T}_{1 ; 2} \equiv \mathcal{I}_{8}^{[3]} \mathcal{P}_{47}^{25}=\frac{z_{38}^{2}}{z_{13}^{2} z_{18}^{2} z_{24} z_{26} z_{28}^{2} z_{34} z_{36}^{2} z_{37} z_{45}^{2} z_{56} z_{57} z_{78}^{2}}$ & 1 \\
$\mathcal{I}_{8}^{[3]} \mathcal{T}_{1 ; 3} \equiv \mathcal{I}_{8}^{[3]} \mathcal{P}_{26}^{13}=\frac{z_{38}^{2}}{z_{13} z_{16} z_{18}^{2} z_{23} z_{25} z_{28}^{2} z_{34} z_{36}^{2} z_{37} z_{45}^{2} z_{47} z_{56} z_{78}^{2}}$ & 2 \\
\hline
\end{tabular}

Figure 41. All the combinations without add new poles of (3.46).

- (1) With $m=1$, we calculate all $6 \times 9$ terms, there are twelve satisfy both Criterion I and II:

$$
\begin{array}{ll}
\mathcal{T}_{2 ; 1}: & \mathcal{P}_{47}^{25} \mathcal{P}_{37}^{26} \mathcal{P}_{38 ; 25}^{13}=\mathcal{P}_{47}^{25} \mathcal{P}_{37}^{26} \mathcal{P}_{38 ; 47}^{13}=\frac{z_{13} z_{25} z_{26} z_{37} z_{47} z_{48}}{z_{14} z_{23} z_{24} z_{38} z_{57} z_{67}} \\
\mathcal{T}_{2 ; 2}: & \mathcal{P}_{47}^{25} \mathcal{P}_{37}^{26} \mathcal{P}_{38 ; 25}^{13}=\mathcal{P}_{47}^{25} \mathcal{P}_{37}^{26} \mathcal{P}_{38 ; 47}^{13}=\frac{z_{13} z_{25} z_{26} z_{37} z_{47} z_{48}}{z_{14} z_{23} z_{24} z_{38} z_{57} z_{67}} \\
\mathcal{T}_{2 ; 3}: & \mathcal{P}_{26}^{13} \mathcal{P}_{47}^{25} \mathcal{P}_{38 ; 25}^{13}=\mathcal{P}_{26}^{13} \mathcal{P}_{47}^{25} \mathcal{P}_{38 ; 47}^{13}=\frac{z_{13}^{2} z_{25} z_{26} z_{47} z_{48}}{z_{14} z_{16} z_{23} z_{24} z_{38} z_{57}} \\
\mathcal{T}_{1 ; 1}: & \mathcal{P}_{37}^{26} \mathcal{P}_{38 ; 25}^{13}=\mathcal{P}_{37}^{26} \mathcal{P}_{38 ; 47}^{13}=\frac{z_{13} z_{26} z_{37} z_{48}}{z_{14} z_{23} z_{38} z_{67}} \\
\mathcal{T}_{1 ; 2}: & \mathcal{P}_{47}^{25} \mathcal{P}_{38 ; 25}^{13}=\mathcal{P}_{47}^{25} \mathcal{P}_{38 ; 47}^{13}=\frac{z_{13} z_{25} z_{47} z_{48}}{z_{14} z_{24} z_{38} z_{57}} \\
\mathcal{T}_{1 ; 3}: & \mathcal{P}_{26}^{13} \mathcal{P}_{38 ; 25}^{13}=\mathcal{P}_{26}^{13} \mathcal{P}_{38 ; 47}^{13}=\frac{z_{13}^{2} z_{26} z_{48}}{z_{14} z_{16} z_{23} z_{38}}
\end{array}
$$

- (2) With $m=2$, we enumerate all $6 \times 36$ that fourteen combinations satisfy both Criterion I and II at the same time. 


$$
\begin{array}{ll}
\mathcal{T}_{2 ; 1}: & \mathcal{P}_{47}^{25} \mathcal{P}_{37}^{26} \mathcal{P}_{38 ; 25}^{13} \mathcal{P}_{38 ; 26}^{13}=\mathcal{P}_{47}^{25} \mathcal{P}_{37}^{26} \mathcal{P}_{38 ; 26}^{13} \mathcal{P}_{38 ; 47}^{13}=\frac{z_{13}^{2} z_{25} z_{26} z_{37} z_{47} z_{48} z_{68}}{z_{14} z_{16} z_{23} z_{24} z_{38}^{2} z_{57} z_{67}} \\
\mathcal{T}_{2 ; 2}: & \mathcal{P}_{47}^{25} \mathcal{P}_{37}^{26} \mathcal{P}_{38 ; 25}^{13} \mathcal{P}_{38 ; 26}^{13}=\mathcal{P}_{47}^{25} \mathcal{P}_{37}^{26} \mathcal{P}_{38 ; 26}^{13} \mathcal{P}_{38 ; 47}^{13}=\frac{z_{13}^{2} z_{25} z_{26} z_{37} z_{47} z_{48} z_{68}}{z_{14} z_{16} z_{23} z_{24} z_{38}^{2} z_{57} z_{67}} \\
\mathcal{T}_{2 ; 3}: & \mathcal{P}_{26}^{13} \mathcal{P}_{47}^{25} \mathcal{P}_{38 ; 25}^{13} \mathcal{P}_{38 ; 26}^{37}=\mathcal{P}_{26}^{13} \mathcal{P}_{47}^{25} \mathcal{P}_{38 ; 47}^{13} \mathcal{P}_{38 ; 26}^{37}=\frac{z_{13}^{2} z_{25} z_{26} z_{37} z_{47} z_{48} z_{68}}{z_{14} z_{16} z_{23} z_{24} z_{38}^{2} z_{57} z_{67}} \\
\mathcal{T}_{1 ; 1}: & \mathcal{P}_{37}^{26} \mathcal{P}_{38 ; 25}^{13} \mathcal{P}_{38 ; 26}^{13}=\mathcal{P}_{37}^{26} \mathcal{P}_{38 ; 26}^{13} \mathcal{P}_{38 ; 47}^{13}=\frac{z_{13}^{2} z_{26} z_{37} z_{48} z_{68}}{z_{14} z_{16} z_{23} z_{38}^{2} z_{67}} \\
\mathcal{T}_{1 ; 2}: & \mathcal{P}_{47}^{25} \mathcal{P}_{38 ; 25}^{13} \mathcal{P}_{38 ; 26}^{13}=\mathcal{P}_{47}^{25} \mathcal{P}_{33 ; 26}^{13} \mathcal{P}_{38 ; 47}^{13}=\frac{z_{13}^{2} z_{25} z_{47} z_{48} z_{68}}{z_{14} z_{16} z_{24} z_{38}^{2} z_{57}} \\
& \mathcal{P}_{47}^{25} \mathcal{P}_{38 ; 25}^{13} \mathcal{P}_{38 ; 26}^{37}=\mathcal{P}_{47}^{25} \mathcal{P}_{38 ; 47}^{13} \mathcal{P}_{38 ; 26}^{37}=\frac{z_{13} z_{25} z_{33} z_{47} z_{48} z_{68}}{z_{14} z_{24} z_{38}^{2} z_{57} z_{67}} \\
\mathcal{T}_{1 ; 3}: & \mathcal{P}_{26}^{13} \mathcal{P}_{38 ; 25}^{13} \mathcal{P}_{38 ; 26}^{37}=\mathcal{P}_{26}^{13} \mathcal{P}_{38 ; 47}^{13} \mathcal{P}_{38 ; 26}^{37}=\frac{z_{13}^{2} z_{26} z_{37} z_{48} z_{68}}{z_{14} z_{16} z_{23} z_{38}^{2} z_{67}}
\end{array}
$$

- (3) With $m=3$, there are two combinations satisfy both Criterion I and II in $6 \times 84$ terms.

$$
\mathcal{T}_{1 ; 2}: \quad \mathcal{P}_{47}^{25} \mathcal{P}_{38 ; 25}^{13} \mathcal{P}_{38 ; 26}^{13} \mathcal{P}_{38 ; 26}^{37}=\mathcal{P}_{47}^{25} \mathcal{P}_{38 ; 26}^{13} \mathcal{P}_{38 ; 47}^{13} \mathcal{P}_{38 ; 26}^{37}=\frac{z_{13}^{2} z_{25} z_{37} z_{47} z_{48} z_{68}^{2}}{z_{14} z_{16} z_{24} z_{38}^{3} z_{57} z_{67}}
$$

- (4) We verified that for $m>3$, there do not exist any combinations that satisfy both Criterion I and II at the same time.

All of above eleven combinations of primary cross-ratio factors give nine different pickfactors:

$$
\begin{aligned}
& \frac{z_{13} z_{25} z_{26} z_{37} z_{47} z_{58}}{z_{15} z_{23} z_{24} z_{38} z_{57} z_{67}} ; \quad \frac{z_{13}^{2} z_{26} z_{58}}{z_{15} z_{16} z_{23} z_{38}} ; \quad \frac{z_{13}^{2} z_{25} z_{26} z_{47} z_{58}}{z_{15} z_{16} z_{23} z_{24} z_{38} z_{57}} ; \quad \frac{z_{13} z_{26} z_{37} z_{58}}{z_{15} z_{23} z_{38} z_{67}} ; \quad \frac{z_{13}^{2} z_{26} z_{37} z_{58} z_{68}}{z_{15} z_{16} z_{23} z_{38}^{2} z_{67}} ; \\
& \frac{z_{13} z_{25} z_{47} z_{58}}{z_{15} z_{24} z_{38} z_{57}} ; \quad \frac{z_{13}^{2} z_{25} z_{47} z_{58} z_{68}}{z_{15} z_{16} z_{24} z_{38}^{2} z_{57}} ; \quad \frac{z_{13}^{2} z_{25} z_{37} z_{47} z_{58} z_{68}^{2}}{z_{15} z_{16} z_{24} z_{38}^{3} z_{57} z_{67}} ; \quad \frac{z_{13}^{2} z_{25} z_{26} z_{37} z_{47} z_{58} z_{68}}{z_{15} z_{16} z_{23} z_{24} z_{38}^{2} z_{57} z_{67}} ;
\end{aligned}
$$

When multiplying each of them to the original CHY-integrand, they all give the same Feynman diagrams containing the pole $s_{1278}$ only:

$$
\begin{aligned}
& \frac{1}{s_{28} s_{36} s_{45} s_{128} s_{1278}}+\frac{1}{s_{18} s_{36} s_{45} s_{178} s_{1278}}+\frac{1}{s_{18} s_{36} s_{45} s_{128} s_{1278}} \\
& +\frac{1}{s_{28} s_{36} s_{45} s_{278} s_{1278}}+\frac{1}{s_{36} s_{45} s_{78} s_{278} s_{1278}}+\frac{1}{s_{36} s_{45} s_{78} s_{178} s_{1278}}
\end{aligned}
$$

\section{Conclusion}

In this note, we have presented two results. In the first result, starting with the Cayley tree type of CHY-integrands, we show how to write down the corresponding effective Feynman 
diagrams. Base on the topological structure of the Cayley tree graph, there are two types of primary effective Feynman vertex: the colour ordered type $V_{C}$ corresponding the line subgraph of Cayley tree and the permutation type $V_{P}$ corresponding to vertex with multiple branches. For the $V_{C}$-type effective vertex, the $\left|V_{C}\left\{\left(l_{1}, l_{2}, \ldots, l_{n}\right)\right\}\right|$ represents all the cubic Feynman diagrams respecting the colour order of the list $\left\{\left(l_{1}, l_{2}, \ldots, l_{n}\right)\right\}$. For the $P$-type vertex $V_{P}\left(k ; \widetilde{K}_{1} ш \widetilde{K}_{2} \amalg \ldots ш \widetilde{K}_{t} ; n\right)$, it represents the comb-like cubic Feynman graph diagrams with ordering from the shuffle algebra. We show how to determine two types of vertexes from the Cayley tree by decomposing the tree into line pieces and vertexes.

Our second result is the construction of picking up factor for general CHY-integrands containing only simple poles. Our algorithm has generalized the result in [18, 19] for the bi-adjoint scalar theories. Unlike the situation of bi-adjoint theory where one needs only a cross-ratio factor, for general CHY-integrand, we need to construct all possible crossratio factors from the linking set Links $[A, \bar{A}]$, including the denominators and numerators. When multiplying them together, we need to introduce two criteria to select the right combination. We demonstrate our algorithm by several non-trivial examples.

Based on results in this note, there are several interesting directions one can investigate. First, we have constructed the picking up factor for selecting a particular pole from all Feynman diagrams. Since one can represent these diagrams using the polytope, it is natural to ask, could we using the geometric picture, i.e., projecting from one dimension higher object to its specific face, to understand and construct the same picking up factor. Secondly, in [20], using the picking pole technique, the one-loop CHY-integrand for general bi-adjoint scalar theory has been constructed by removing singular poles (i.e., tadpoles and massless bubbles). For general theory, such as Gravity and Yang-Mills, the CHY-integrands are general. Thus one can try if it is possible to construct the one-loop integrands for these general theory by removing singular contribution using the picking up factor developed in this note. Thirdly, it is well known the general CHY-integrand could contain higher-order poles. The standard method to deal with them is given in [21] by decomposing them as a linear combination of CHY-integrands with only simple poles using cross-ratio identity. It is interesting to ask if our picking pole factor provides another way to reduce higher poles to lower poles.

Fourthly, in this paper we considered only the effective Feynman Diagram to CHYintegrand of $m(C \mid C)$ type and have seen the connection between polytopes and effective Feynman Diagrams. However, for the $m\left(C_{1} \mid C_{2}\right)$, as pointed out in [1], it is the intersection of $m\left(C_{1} \mid C_{1}\right)$ and $m\left(C_{2} \mid C_{2}\right)$. This point can also easily seen from the effective Feynman diagrams. In $[22,23]$, they pointed out that such pairing is essentially the same thing as the intersection pairing of two cocycles in a certain cohomology theory. Thus it is an interesting direction to investigate the symmetry of the intersection between two effective Feynman diagrams and the corresponding geometric representation.

\section{Acknowledgments}

This work is supported by Qiu-Shi Funding and the National Natural Science Foundation of China (NSFC) with Grant No.11935013, No.11575156. 
Open Access. This article is distributed under the terms of the Creative Commons Attribution License (CC-BY 4.0), which permits any use, distribution and reproduction in any medium, provided the original author(s) and source are credited.

\section{References}

[1] X. Gao, S. He and Y. Zhang, Labelled tree graphs, Feynman diagrams and disk integrals, JHEP 11 (2017) 144 [arXiv:1708.08701] [INSPIRE].

[2] F. Cachazo, S. He and E.Y. Yuan, Scattering of Massless Particles in Arbitrary Dimensions, Phys. Rev. Lett. 113 (2014) 171601 [arXiv:1307.2199] [InSPIRE].

[3] F. Cachazo, S. He and E.Y. Yuan, Scattering of Massless Particles: Scalars, Gluons and Gravitons, JHEP 07 (2014) 033 [arXiv:1309.0885] [INSPIRE].

[4] F. Cachazo, S. He and E.Y. Yuan, Scattering equations and Kawai-Lewellen-Tye orthogonality, Phys. Rev. D 90 (2014) 065001 [arXiv:1306.6575] [InSPIRE].

[5] F. Cachazo, S. He and E.Y. Yuan, Scattering Equations and Matrices: From Einstein To Yang-Mills, DBI and NLSM, JHEP 07 (2015) 149 [arXiv:1412.3479] [INSPIRE].

[6] F. Cachazo, S. He and E.Y. Yuan, Einstein-Yang-Mills Scattering Amplitudes From Scattering Equations, JHEP 01 (2015) 121 [arXiv:1409.8256] [INSPIRE].

[7] L. Dolan and P. Goddard, The Polynomial Form of the Scattering Equations, JHEP 07 (2014) 029 [arXiv: 1402.7374] [INSPIRE].

[8] C. Kalousios, Scattering equations, generating functions and all massless five point tree amplitudes, JHEP 05 (2015) 054 [arXiv: 1502.07711] [INSPIRE].

[9] R. Huang, J. Rao, B. Feng and Y.-H. He, An Algebraic Approach to the Scattering Equations, JHEP 12 (2015) 056 [arXiv:1509. 04483] [INSPIRE].

[10] M. Søgaard and Y. Zhang, Scattering Equations and Global Duality of Residues, Phys. Rev. D 93 (2016) 105009 [arXiv: 1509.08897] [INSPIRE].

[11] L. Dolan and P. Goddard, General Solution of the Scattering Equations, JHEP 10 (2016) 149 [arXiv: 1511.09441] [INSPIRE].

[12] C. Cardona and C. Kalousios, Comments on the evaluation of massless scattering, JHEP 01 (2016) 178 [arXiv: 1509.08908] [INSPIRE].

[13] C. Cardona and C. Kalousios, Elimination and recursions in the scattering equations, Phys. Lett. B 756 (2016) 180 [arXiv: 1511.05915] [INSPIRE].

[14] C. Baadsgaard, N.E.J. Bjerrum-Bohr, J.L. Bourjaily and P.H. Damgaard, Integration Rules for Scattering Equations, JHEP 09 (2015) 129 [arXiv:1506.06137] [INSPIRE].

[15] C. Baadsgaard, N.E.J. Bjerrum-Bohr, J.L. Bourjaily and P.H. Damgaard, Scattering Equations and Feynman Diagrams, JHEP 09 (2015) 136 [arXiv:1507.00997] [INSPIRE].

[16] C. Baadsgaard, N.E.J. Bjerrum-Bohr, J.L. Bourjaily, P.H. Damgaard and B. Feng, Integration Rules for Loop Scattering Equations, JHEP 11 (2015) 080 [arXiv:1508.03627] [INSPIRE].

[17] C. Cardona, B. Feng, H. Gomez and R. Huang, Cross-ratio Identities and Higher-order Poles of CHY-integrand, JHEP 09 (2016) 133 [arXiv: 1606.00670] [INSPIRE]. 
[18] R. Huang, F. Teng and B. Feng, Permutation in the CHY-Formulation, Nucl. Phys. B 932 (2018) 323 [arXiv: 1801.08965] [inSPIRE].

[19] B. Feng, CHY-construction of Planar Loop Integrands of Cubic Scalar Theory, JHEP 05 (2016) 061 [arXiv: 1601.05864] [INSPIRE].

[20] B. Feng and C. Hu, One-loop CHY-Integrand of Bi-adjoint Scalar Theory, JHEP 02 (2020) 187 [arXiv: 1912.12960] [INSPIRE].

[21] R. Huang, B. Feng, M.-x. Luo and C.-J. Zhu, Feynman Rules of Higher-order Poles in CHY Construction, JHEP 06 (2016) 013 [arXiv: 1604.07314] [INSPIRE].

[22] S. Mizera, Scattering Amplitudes from Intersection Theory, Phys. Rev. Lett. 120 (2018) 141602 [arXiv: 1711.00469] [INSPIRE].

[23] H. Frost, Biadjoint scalar tree amplitudes and intersecting dual associahedra, JHEP 06 (2018) 153 [arXiv: 1802.03384] [INSPIRE]. 